\title{
The contribution by luminous blue variable stars to the dust content of the Magellanic Clouds *,
}

\author{
C. Agliozzo ${ }^{1}$, N. Phillips ${ }^{1}$, A. Mehner ${ }^{2}$, D. Baade ${ }^{1}$, P. Scicluna 2 , F. Kemper ${ }^{1,3}$, D. Asmus ${ }^{2,4,5}$, W.-J. de Wit ${ }^{2}$, G. \\ Pignata ${ }^{6,7}$
}

\author{
1 European Southern Observatory, Karl-Schwarzschild-Strasse 2, 85748 Garching bei München, Germany \\ e-mail: claudia.agliozzodeso.org \\ 2 European Southern Observatory, Alonso de Cordova 3107, Vitacura, Santiago de Chile, Chile \\ 3 Institute of Astronomy and Astrophysics, Academia Sinica, No. 1, Sec. 4, Roosevelt Rd., Taipei 10617, Taiwan \\ 4 School of Physics \& Astronomy, University of Southampton, Southampton SO17 1BJ, UK \\ 5 Gymnasium Schwarzenbek, Buschkoppel 7, 21493 Schwarzenbek, Germany \\ 6 Departamento de Ciencias Fisicas, Universidad Andres Bello, Avda. Republica 252, Santiago, 8320000, Chile \\ 7 Millennium Institute of Astrophysics (MAS), Nuncio Monseñor Sótero Sanz 100, Providencia, Santiago, Chile
}

\begin{abstract}
Context. Previous studies have concluded that low- and intermediate-mass stars cannot account for the interstellar dust yield in the Magellanic Clouds inferred from far-infrared and sub-millimetre observations.

Aims. Luminous blue variable stars (LBVs) form dust as a result of episodic, violent mass loss. To investigate their contribution as dust producers in the Magellanic Clouds, we analyse 31 confirmed and candidate LBVs from a recent census.

Methods. We built a maximally complete multi-wavelength dataset of these sources from archival space telescope images and catalogues from near-infrared to millimetre wavelengths. We also present new Very Large Telescope VISIR observations of three sources in the Large Magellanic Cloud (LMC). We review the LBV classification on the basis of the infrared spectral energy distribution. To derive characteristic dust parameters, we fitted the photometry resulting from a stacking analysis, which consists of co-adding images of the same wavelength band of several targets to improve the signal-to-noise. For comparison we also stacked the images of low- and intermediate-mass evolved stars in the LMC.

Results. We find four classes of sources: 1) LBVs showing mid-infrared dust emission plus near-infrared free-free emission from an ionised stellar wind (Class 1a) or only mid-infrared dust emission (Class 1b); 2) LBVs with a near-infrared excess due to free-free emission only (Class 2); 3) objects with an $\mathrm{sgB}[\mathrm{e}]$ classification in the literature, displaying a distinctive hot dust component; and 4) objects with no detected stellar winds and no circumstellar matter in their SEDs. From the stacking analysis of the 18 Class 1 and 2 objects in the LMC, we derived an integrated dust mass of $0.11_{-0.03}^{+0.06} M_{\odot}$. This is two orders of magnitude larger than the value inferred from stacking 1342 extreme-asymptotic giant branch (AGB) stars. The dust mass of individual LBVs does not correlate with the stellar parameters, possibly suggesting that the dust production mechanism is independent of the initial stellar mass or that the stars have different evolutionary histories. The total dust yield from LBVs over the age of the LMC is $\sim 10^{4}-10^{5} M_{\odot}$. The one order of magnitude uncertainty is mainly due to uncertainties of the LBV population, star formation history, and initial mass function. Conclusions. LBVs are potentially the second most important source of dust in normal galaxies. The role of dust destruction in LBV nebulae by a possible subsequent supernova $(\mathrm{SN})$ blast wave has yet to be determined. Recent theoretical developments in the field of dust processing by SN shocks highlight the potential survival of dust grains from the pre-existing circumstellar nebula.
\end{abstract}

Key words. Stars: mass-loss - Stars: variables: S Doradus - (ISM:) dust, extinction - Galaxies: ISM - (Galaxies:) Magellanic Clouds

\section{Introduction}

The origin of dust on cosmic scales is of great astrophysical interest. The cosmic importance of dust is illustrated by the fact that about $50 \%$ of starlight is reprocessed by dust (Dole et al. 2006). Thus, dust dominates how we see galaxies. Dust is very important for the thermal balance of the interstellar medium (ISM) of galaxies and accordingly for star formation (McKinnon et al. 2016, and references therein). Large amounts of dust have been observed in high redshift galaxies $(z \gtrsim 6$, e.g. Riechers et al. 2017; Laporte et al. 2017), where low- and intermediatemass stars did not have time to evolve and thus to inject dust

* Based on observations made with ESO Telescopes at the La Silla Paranal Observatory under programme IDs: 097.D-0612(A,B) and 0100.D-0469(A,B). into the ISM. It is widely accepted now that dust must arise from rapidly evolving (1-10 Myr) massive stars, particularly in the ejecta of core-collapse supernovae (CC-SNe) (Sugerman et al. 2006). In fact, large amounts of dust $\left(0.1-1 M_{\odot}\right)$ were postulated by Todini \& Ferrara (2001) and Nozawa et al. (2003), under the assumption of favourable conditions in the SN ejecta. Nevertheless, for nearby CC-SNe, the dust masses inferred from mid-infrared observations are in general of the order of $10^{-4}-$ $10^{-2} M_{\odot}$ (e.g. Kotak et al. 2009, Fox et al. 2011). Dust masses of the order of $10^{-1} M_{\odot}$ were reported from sub-millimetre observations of SN1987A (e.g. Indebetouw et al. 2014; Matsuura et al. 2015), suggesting that perhaps cold dust emission is not captured by mid-infrared observations. However, such estimates are difficult to compare because they are often based on different observational constraints and assumptions. It is also expected 
that only a fraction of the newly formed dust reaches the ISM, as it will be heavily processed and destroyed by the reverse shock (see Micelotta et al. 2018, Gall \& Hjorth 2018). Dust can also grow in dense ISM from existing dust grains (e.g. Draine 2009), although this is still not sufficient, together with the contribution from $\mathrm{SNe}$, to explain dust yields in galaxies (e.g. Tamura et al. 2019). The topic is also controversial with respect to the nearby Magellanic Clouds (MCs).

The MCs are two irregular dwarf galaxies with metallicities 0.5 and 0.25 times Solar for the Large Magellanic Cloud (LMC) and the Small Magellanic Cloud (SMC), respectively. Their relative proximity to the Milky Way (MW), accurate distance measurements and low line-of-sight extinction make them an ideal laboratory, resembling the physical conditions of galaxies at the peak of star formation. In the past decades, several efforts were made to estimate dust yields in these galaxies and determine the primary dust sources at low metallicities. With the advent of Herschel this became possible. Particularly the HERITAGE Herschel key project (Meixner et al. 2013), observing in five bands from 100 to $500 \mu \mathrm{m}$, offered a plethora of data that advanced our understanding of the dust budget problem. Recent estimates report integrated dust masses of $(7.3 \pm 1.7) \times 10^{5}$ and $(8.3 \pm 2.1) \times 10^{4} M_{\odot}$ for the LMC and SMC, respectively (Gordon et al. 2014), although later Chastenet et al. (2017) derived dust masses about a factor of two smaller. Several teams tried to identify the main sources of dust in such galaxies. Srinivasan et al. (2009), Matsuura et al. (2009), Riebel et al. (2012), Boyer et al. (2012) and Srinivasan et al. (2016) studied the dust production rate from low- and intermediate-mass stars in the MCs using mid-infrared observations. Jones et al. (2015) identified some tens of new evolved stellar objects from HERITAGE. These works generally agree on the result that the global dust production from the numerous population of asymptotic giant branch (AGB) and red supergiant (RSG) stars cannot account for the ISM dust reported from far-infrared and sub-millimetre observations. Another result is that the majority of dust input from low- and intermediate-mass stellar sources comes from "extreme" AGB (Srinivasan et al. 2009, Riebel et al. 2012, Boyer et al. 2012).

Spitzer observations from the SAGE legacy survey (Meixner et al. 2006) were used by Bonanos et al. (2009, 2010) to investigate the infrared properties of massive stars. They found an excess of emission at $24 \mu \mathrm{m}$ due to dust from a handful of luminous blue variable (LBV) stars. Some of these, and other LBVs with known extended nebulae detected with HST (Weis 2003), were then followed up and large masses of dust were measured (Guha Niyogi et al. 2014, Agliozzo et al.|2017a|b, 2019).

In the classical scenario, LBVs are transitional objects from the main sequence of $\mathrm{O}, \mathrm{B}$ stars to the Wolf-Rayet (WR) phase (Conti 1984). This represents a short phase of evolution of the most massive $\left(M \geq 25 M_{\odot}\right)$ stars, ranging in duration from $10^{4}$ to $10^{5} \mathrm{yr}$ (e.g. Maeder 1989, Massey et al. 2007; Smith 2014). Observationally, LBVs are blue supergiants (BSGs) that experience instabilities, observed in the form of spectroscopic and photometric variabilities, usually in the optical and near-infrared. These instabilities are poorly understood but, on the basis of their observational properties, are phenomenologically divided into three main groups: giant eruptions, S Doradus-type variability, and micro-variabilities (Humphreys \& Davidson 1994, van Genderen 2001). Although the physical mechanisms underlying these instabilities are possibly different (e.g. the vicinity to the Eddington limit, sub-photospheric instabilities, bistability jump, envelope inflation, wind-envelope interaction, fast rotation, binarity, stellar merger; Humphreys \& Davidson 1994, Gallagher
1989, Vink et al. 1999, Groh et al. 2009, Gräfener et al. 2012, Portegies Zwart \& van den Heuvel 2016, Owocki et al. 2017, Grassitelli et al.|2021), observations suggest increases in the mass-loss rates by about a factor of three in S Doradus-type variability (e.g. Vink \& de Koter 2002) or by several orders of magnitude in giant eruptions, like that of $\eta$ Carinae (e.g. Humphreys \& Davidson 1994). During the quiescent state, LBV mass-loss rates range between $10^{-7}$ and $10^{-5} M_{\odot} \mathrm{yr}^{-1}$, typical of BSGs as explained by the radiatively-driven stellar-wind theory (Castor et al. 1975; Sundqvist et al. 2019).

Particularly enigmatic are the rare giant eruptions, initially often mistaken for CC-SNe, because of their brightness, and subsequently named SN impostors (e.g. Van Dyk \& Matheson 2012, Mauerhan et al.|2013; Tartaglia et al. 2016, Pastorello et al.|2017, Elias-Rosa et al.|2018; Reguitti et al.2019). Famous examples in our Galaxy are $\eta$ Car and P Cygni. Observationally, mass-loss rates exceeding $10^{-3}-10^{-2} M_{\odot} \mathrm{yr}^{-1}$ are estimated (e.g. Smith 2014). In the case of $\eta \mathrm{Car}$, more than $40 M_{\odot}$ (Morris et al. 2017) were released during the Great Eruption in the XIX century, which lasted about 20 years, forming the Homunculus nebula. In eruptions of SN impostors, nebular masses of the order of $0.1 M_{\odot}$ are observed (e.g. Margutti et al. 2014). Several observational and theoretical works suggest that some LBVs could be the immediate progenitors of SNe (e.g. Kotak \& Vink 2006, Trundle et al. 2008: Gal-Yam \& Leonard 2009;:Smith et al. 2007 2008a, Groh et al. 2013; Groh 2014; Boian \& Groh 2018).

Luminous blue variable stars are often surrounded by dusty circumstellar nebulae. In the Milky Way typical dust masses ranging between $10^{-3}$ and $10^{-1} M_{\odot}$ are reported (Hutsemekers 1997, Umana et al. 2012, Vamvatira-Nakou et al. 2013, 2015, Agliozzo et al. 2014, Lau et al. 2014; Arneson et al. 2018). Furthermore, several tens of candidate LBVs were identified on the basis of the detection of infrared circumstellar nebulae (Egan et al. 2002; Clark et al. 2003, Gvaramadze et al. 2010) and of an infrared excess usually peaking between 24 and $70 \mu \mathrm{m}$. The central star, which is a BSG during quiescence, is very hot, and one does not expect dust condensation in its wind. However, the physical conditions in the optically thick and cool "pseudo-photospheres" formed during eruptions are favourable for dust condensation and growth (Kochanek 2011b). Kochanek showed analytically that the required temperature and particle density for dust formation are met for mass-loss rates larger than $10^{-2.5} M_{\odot} \mathrm{yr}^{-1}$, that are typical of giant eruptions. Grain growth and dust evolution may be observed in S Doradus variables (which have mass-loss rates that are a couple of orders of magnitudes lower), as suggested by the disappearance of the silicate bump around $10-13 \mu \mathrm{m}$ during the most recent $\mathrm{S}$ Dor outburst of the Magellanic LBV RMC 71 (Mehner et al. 2017). Another way to form dust around a massive star is in the colliding winds of close binaries, as in some Wolf Rayet stars. In these systems dust seems to form episodically, near the periastron passage, or persistently, creating pinwheel-like nebulae (e.g. Crowther 2007, Lau et al. 2020). This mechanism of dust formation is presumably the case of the Galactic LBV binary HR Car (Boffin et al. 2016), a model of which to explain the clumpy and dusty inner nebula is an Archimedean spiral centred on the binary system (alternative to the expanding bipolar lobe model, see Buemi et al.2017, and references therein). Episodic dust formation also potentially occurs in the shocked regions of $\eta$ Car's binary colliding winds (Smith 2010).

In the MCs only up to one third of the LBV sample appears in studies of the primary dust sources (see above), and the dust masses from these objects remain unconstrained. Here we aim to expand these studies to the full population of LBVs in the MCs. 


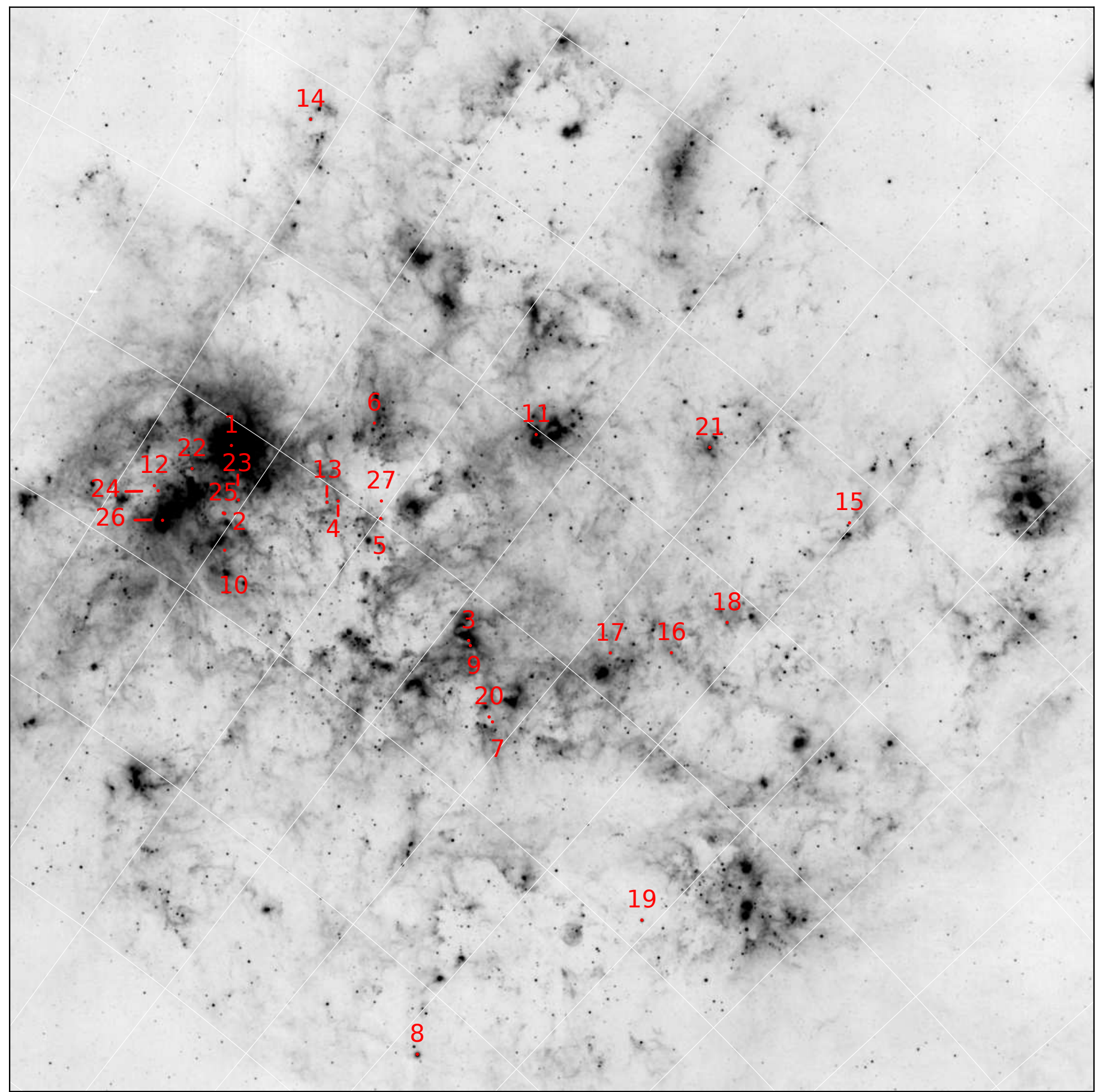

Fig. 1: Cutout of the SAGE MIPS24 image (Meixner et al. 2006) of the LMC and positions of the candidate and confirmed LBVs analysed in this work. The numbers are the IDs in Table 1 , and the red circles have a radius of $30^{\prime \prime}$. North is towards the top right.

Figs. 1 and 2 show the locations of the candidate and confirmed LBVS in the LMC and SMC that are analysed in this work. We have built a maximally complete multi-wavelength dataset of 31 LBVs mostly from archival space telescope images and catalogues, but also have included ground-based mid-infrared observations of three sources. In addition, we derived new photometric values for sources missing in catalogues or with low-accuracy photometry (Sects. 2 and 3). The goals of this work are:

1. Review the status of the MC LBV sample by means of their infrared properties, combined with literature information (Sect. 4).
2. Estimate the dust masses of LBVs in the MCs by modelling individual sources (Sect. 5.1) and modelling photometry from stacked images of the LBVs in the LMC (Sect.5.2).

3. Evaluate the importance of LBVs in producing dust by comparing the derived dust mass with that inferred from the stacking of lower-mass stars and with literature values of ISM dust yields (Sects. 5.3 and 6.

\section{The multiwavelength dataset}

The sample was extracted from the most recent census of LBVs in the Local Group (Richardson \& Mehner 2018), which con- 
Table 1: Sources, celestial coordinates (Gaia Collaboration 2018), LBV status (previous: Richardson \& Mehner 2018, new: this work), and SED-based classification proposed in this work.

\begin{tabular}{|c|c|c|c|c|c|c|c|}
\hline \multirow[t]{2}{*}{ ID } & \multirow[t]{2}{*}{ Name 1} & \multirow[t]{2}{*}{ Name 2} & \multirow[t]{2}{*}{ RA (ICRS, J2000) } & \multirow[t]{2}{*}{ Dec (ICRS, J2000) } & \multicolumn{2}{|c|}{ LBV status } & \multirow{2}{*}{$\begin{array}{l}\text { SED } \\
\text { Class }\end{array}$} \\
\hline & & & & & Previous & New & \\
\hline 1 & RMC 143 & CPD -69463 & 053851.617 & -690807.31 & LBV & LBV & 1 \\
\hline 2 & RMC 127 & HD 269858 & 053643.694 & -692947.45 & LBV & LBV & 1 \\
\hline 3 & SDoradus & HD 5343 & 051814.357 & -691501.15 & LBV & LBV & 1 \\
\hline 4 & RMC 110 & HD 269662 & 053051.476 & -690258.59 & LBV & LBV & 1 \\
\hline 5 & HD 269582 & LHA $120-S 83$ & 052752.662 & -685908.49 & LBV & LBV & 2 \\
\hline 6 & RMC 116 & HD 269700 & 053152.282 & -683238.86 & LBV & LBV & 2 \\
\hline 7 & HD 269216 & LHA $120-S 88$ & 051330.781 & -693223.65 & LBV & LBV & 1 \\
\hline 8 & RMC 71 & HD 269006 & 050207.394 & -712013.12 & LBV & LBV & 1 \\
\hline 9 & RMC 85 & HD 269321 & 051756.074 & -691603.81 & LBV & LBV & - \\
\hline 10 & RMC 123 & HD 37836 & 053516.633 & -694038.44 & cLBV & cLBV & 2 \\
\hline 11 & RMC99 & HD 269445 & 052259.785 & -680146.63 & cLBV & cLBV & 2 \\
\hline 12 & Sk - 69279 & - & 054144.656 & -693514.90 & cLBV & cLBV & 1 \\
\hline 13 & LHA 120-S 119 & HD 269687 & 053125.525 & -690538.56 & cLBV & cLBV & 1 \\
\hline 14 & LHA 120-S 61 & AL 418 & 054551.939 & -671425.94 & cLBV & cLBV & 1 \\
\hline 15 & RMC 74 & HD 268939 & 050414.909 & -671505.25 & cLBV & cLBV & 2 \\
\hline 16 & RMC 78 & HD 269050 & 050720.422 & -683208.57 & cLBV & cLBV & 1 \\
\hline 17 & RMC 81 & HD 269128 & 051022.789 & -684623.82 & cLBV & cLBV & 1 \\
\hline 18 & LHA $120-S 18$ & $\mathrm{Sk}-6842$ & 050553.981 & -681050.54 & cLBV & cLBV & 1 \\
\hline 19 & RMC 66 & HD 268835 & 045647.080 & -695024.77 & cLBV & $\operatorname{sgB}[\mathrm{e}]^{b}$ & 3 \\
\hline 20 & RMC 84 & HD 269227 & 051354.280 & -693146.66 & cLBV & $\operatorname{sgB}[\mathrm{e}]^{c}$ & 3 \\
\hline 21 & HD 34664 & $S k-6764$ & 051352.994 & -672654.82 & cLBV & $\operatorname{sgB}[\mathrm{e}]^{b}$ & 3 \\
\hline 22 & HD 38489 & $S k-69259$ & 054013.321 & -692246.49 & cLBV & $\operatorname{sgB}[\mathrm{e}]^{b}$ & 3 \\
\hline 23 & RMC 126 & HD 37974 & 053625.854 & -692255.79 & cLBV & $\operatorname{sgB}[\mathrm{e}]^{b}$ & 3 \\
\hline 24 & $\mathrm{Sk}-69271$ & CPD - 69500 & 054120.126 & -693622.89 & cLBV & $\mathrm{YSG}^{d}$ & 4 \\
\hline 25 & RMC 128 & HD 269859 & 053647.188 & -692952.09 & cLBV & $\mathrm{B} 2 \mathrm{Ia}^{e}$ & 4 \\
\hline 26 & RMC 149 & $\mathrm{Sk}-69257$ & 053958.745 & -694404.07 & cLBV & $08.5 \mathrm{II}((\mathrm{f}))^{f}$ & 4 \\
\hline 27 & HD 269604 & Sk -6893 & 052831.367 & -685355.75 & cLBV & A1 Ia-0 ${ }^{g}$ & 4 \\
\hline 28 & RMC 14 & HD5980 & 005926.585 & -720953.93 & LBV & LBV & 2 \\
\hline 29 & RMC40 & HD 6884 & 010718.218 & -722803.66 & LBV & LBV & 1 \\
\hline 30 & RMC 4 & LHA 115-S 6 & 004655.030 & -730834.14 & cLBV & cLBV & 3 \\
\hline 31 & LHA $115-S 18$ & - & 005409.542 & -724143.29 & cLBV & cLBV & 3 \\
\hline
\end{tabular}

Notes. The sample includes confirmed LBV and candidate LBV (cLBV) stars in the MCs. ${ }^{(a)}$ Class 1: sources with a warm/cool dusty nebula peaking in the mid- to far-infrared. Class 2: sources displaying from 1 to $\sim 24 \mu$ m only free-free emission from the stellar wind in addition to the stellar photosphere. Class 3: sources with an SED dominated at all wavelengths $\gtrsim 2 \mu \mathrm{m}$ by a hot dusty component. Class 4: featureless sources only displaying the stellar photosphere. ${ }^{(b)}$ Zickgraf et al. (1986). ${ }^{(c)}$ Stahl et al. (1984). ${ }^{(d)}$ Neugent et al. (2012). ${ }^{(e)}$ Fitzpatrick (1991). ${ }^{(f)}$ Fariña et al. (2009). (g) Osmer (1973).

tains 31 objects in the MCs. Table 1 includes the names and celestial coordinates of all the stars included in this work. More information on individual sources is provided in Sect. 4 .

\subsection{VISIR observations}

RMC71, HD38489, and HD34664 were observed in 2016 and 2017 (ESO program IDs: 097.D-0612(A,B) and 0100.D0469(A,B)) with the upgraded Vlt Imager and Spectrometer for the mid-InfraRed (VISIR; Lagage et al. 2004, Käufl et al. 2015; Kerber et al. 2016) on the Very Large Telescope (VLT, Enard 1991). Some of the data of RMC71 from program 097.D-0612 were previously published in Mehner et al. (2017). The images were taken in standard imaging mode through filters J8.9 (8.7 \pm $0.37 \mu \mathrm{m})$, PAH2_2 $(11.88 \pm 0.19 \mu \mathrm{m})$ and Q1 $(17.65 \pm 0.42 \mu \mathrm{m})$ with perpendicular nodding and a chop/nod throw of $20^{\prime \prime}$ in 097.D-0612 and 10" in 0100.D-0469, respectively. The individual observations are listed in Table 2. J8.9 was used for target acquisition only (hence the short exposure times). For flux calibration and point spread function (PSF) reference, the science observations were preceded or followed by the observation of mid-infrared standard stars (Cohen et al. 1999), which provide a systematic flux uncertainty of $\leq 10 \%$. The data were reduced with the custom Python tool VISIR and ISAAC Pipeline Environmen ${ }^{1}$ (VIPE). Fluxes and source sizes were measured with Gaussian-fitting photometry performed as part of the VIPE reduction.

Both epochs of the PAH2_2 observations of HD 34664 were affected by a chopping-induced PSF instability at the VLT, leading to an artificial elongation of the source. However, in the J8.9 and Q1 filters, the object is consistent with being unresolved $\left(\mathrm{FWHM} \leq 0.4^{\prime \prime}\right.$ in $\mathrm{J} 8.9$ and $\leq 0.5^{\prime \prime}$ in Q1).

The PAH2_2 observation of HD 38489 suffers from the same PSF-instability problem while, during the Q1 observation, the source was offset such that it was on the detector in only half of the chopping positions. The latter fact has been taken into account during the data reduction. Like HD34664, HD 38489 is

1 https://github.com/danielasmus/vipe 
Table 2: Journal of VISIR observations.

\begin{tabular}{llccccc}
\hline \hline Source & Filter & UT start time & Airmass & $\begin{array}{c}\text { IQ at } \lambda \\
(\operatorname{arcsec})\end{array}$ & $\begin{array}{c}\text { Exposure } \\
(\mathrm{s})\end{array}$ & $\begin{array}{c}F_{V} \\
(\mathrm{mJy})\end{array}$ \\
\hline \multirow{3}{*}{ RMC 71 } & J8.9 & $2017-10-0707: 47$ & 1.47 & N/A & 90 & $136 \pm 19$ \\
& J8.9 & $2017-10-1107: 30$ & 1.47 & 0.42 & 90 & $122 \pm 12$ \\
& PAH2_2 & $2016-08-1408: 57$ & 1.63 & 0.35 & 1892 & $408 \pm 41$ \\
& PAH2_2 & $2016-08-1808: 31$ & 1.65 & 0.36 & 1892 & $482 \pm 48$ \\
& PAH2_2 & $2016-09-0508: 11$ & 1.57 & 0.35 & 1892 & $408 \pm 41$ \\
& Q1 & $2016-08-1708: 58$ & 1.64 & 0.46 & 588 & $3261 \pm 326$ \\
& Q1 & $2017-10-0707: 56$ & 1.46 & 0.46 & 1980 & $3207 \pm 321$ \\
& Q1 & $2017-10-1107: 35$ & 1.46 & 0.47 & 1980 & $3490 \pm 349$ \\
HD 34664 & J8.9 & $2017-10-0807: 49$ & 1.38 & 0.29 & 90 & $1051 \pm 105$ \\
& PAH2_2 & $2016-09-0508: 54$ & 1.43 & 0.38 & 1260 & $944 \pm 94$ \\
& PAH2_2 & $2016-09-0809: 02$ & 1.40 & 0.38 & 1260 & $903 \pm 90$ \\
& Q1 & $2017-10-0807: 55$ & 1.37 & 0.46 & 1980 & $580 \pm 58$ \\
& & & & & & \\
HD 38489 & J8.9 & $2017-10-2707: 33$ & 1.41 & N/A & 90 & $905 \pm 129$ \\
& PAH2_2 & $2016-09-2209: 05$ & 1.42 & 0.32 & 990 & $781 \pm 78$ \\
& Q1 & $2017-10-2707: 54$ & 1.41 & 0.46 & 1980 & $561 \pm 56$ \\
\hline
\end{tabular}

Notes. IQ at $\lambda$ gives the approximate image quality at the observing wavelength at the zenith as measured from the flux standard stars observed before or after the science targets. Filter centre wavelengths are J8.9: 8.7 $\mu \mathrm{m}, \mathrm{PAH} 2 \_2: 11.68 \mu \mathrm{m}, \mathrm{Q} 1: 17.65 \mu \mathrm{m}$.

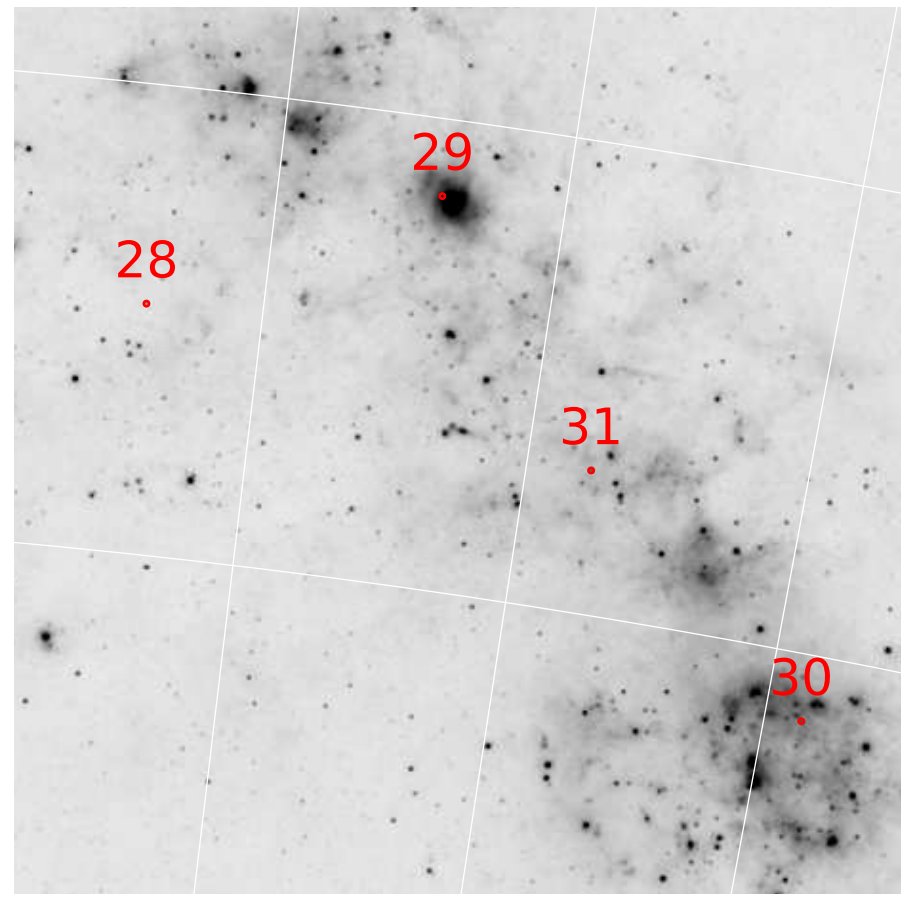

Fig. 2: Cutout of the SAGE MIPS24 image (Meixner et al. 2006) of the SMC and positions of the candidate and confirmed LBVS analysed in this work. The numbers are the IDs in Table 1, and the red circles have a radius of $30^{\prime \prime}$. North is up and east is left.

not obviously resolved, with upper limits in FWHM of $\leq 0.37^{\prime \prime}$ in $\mathrm{J} 8.9$ and $\leq 0.5^{\prime \prime}$ in Q1, respectively.

RMC71 appears resolved in all images, with the following FWHM dimensions: $\sim 0.46^{\prime \prime} \times 0.42^{\prime \prime}$ in J8.9 (PA $~ 126 \mathrm{deg}$ ), $\sim 0.96^{\prime \prime} \times 0.83^{\prime \prime}$ in PAH2_2 (PA $\left.\sim 110 \mathrm{deg}\right)$, and $\sim 0.87^{\prime \prime}$ round in Q1. These are to be regarded as upper limits because none of the observations was made under diffraction-limited condi- tions (also because of the low declination of the object). Thus the differences between the two axes in each filter are considered insignificant. However, the extended nature of this source is robust, because of the consistent appearance at both epochs, including the position angle, and the significantly larger FWHM than found for the calibrator stars. The circumstellar environment of RMC71 appears in agreement with a broad circular shell and an asymmetric core, which is offset from the centre by $\sim 0.2^{\prime \prime}$ toward the north-west (Fig. 3).

\subsection{Archival data}

We consulted the infrared catalogues with the CDS VizieR (Ochsenbein et al. 2000) and IRSA Gator ${ }^{2}$ tools and retrieved photometry from the 2MASS Point Source Catalogue (Cutri et al. 2003), the Spitzer SAGE legacy survey (Meixner et al. 2006 Data Release 3/final), the "Optically bright post-AGB population of LMC" (van Aarle et al. 2011) and "Spitzer Atlas of Stellar Spectra" (Ardila et al.2010) catalogues, the AKARI IRC all-sky survey and LMC point source catalogues (Ishihara et al. 2010, Kato et al. 2012), WISE surveys (Cutri et al. 2012), the MSX Point Source Catalogue (Egan et al. 2003), and Herschel HERITAGE survey (Meixner et al. 2013). We evaluated all stars for possible contamination by neighbouring sources by visually inspecting the infrared images when available (for each source, these are shown in Figs. B.1, B.2, B.3, B.5, B.6 of Appendix B.

We checked for ALMA observations of the sample. ALMA Band 7 (343 GHz) observations of RMC 127, RMC 143 and LHA 120-S61 were previously reported in Agliozzo et al. (2017a b, 2019). Unpublished ALMA/ACA data of a mosaic region containing RMC 14 are available in the archive, in Bands 3 ( 93 GHz; project ID: 2018.A.00049.S) and 6 ( 220 GHz; project ID: 2017.A.00054.S). RMC 14 is not detected, with $3 \sigma$ upper limits of $1.41 \mathrm{mJy}$ at $93 \mathrm{GHz}$ and $6.0 \mathrm{mJy}$ at $220 \mathrm{GHz}$. We also made use of the SPT+Planck maps of the MCs at $1.4 \mathrm{~mm}$

$2 \longdiv { \text { https://irsa.ipac.caltech.edu/applications/ } }$ Gator/ 

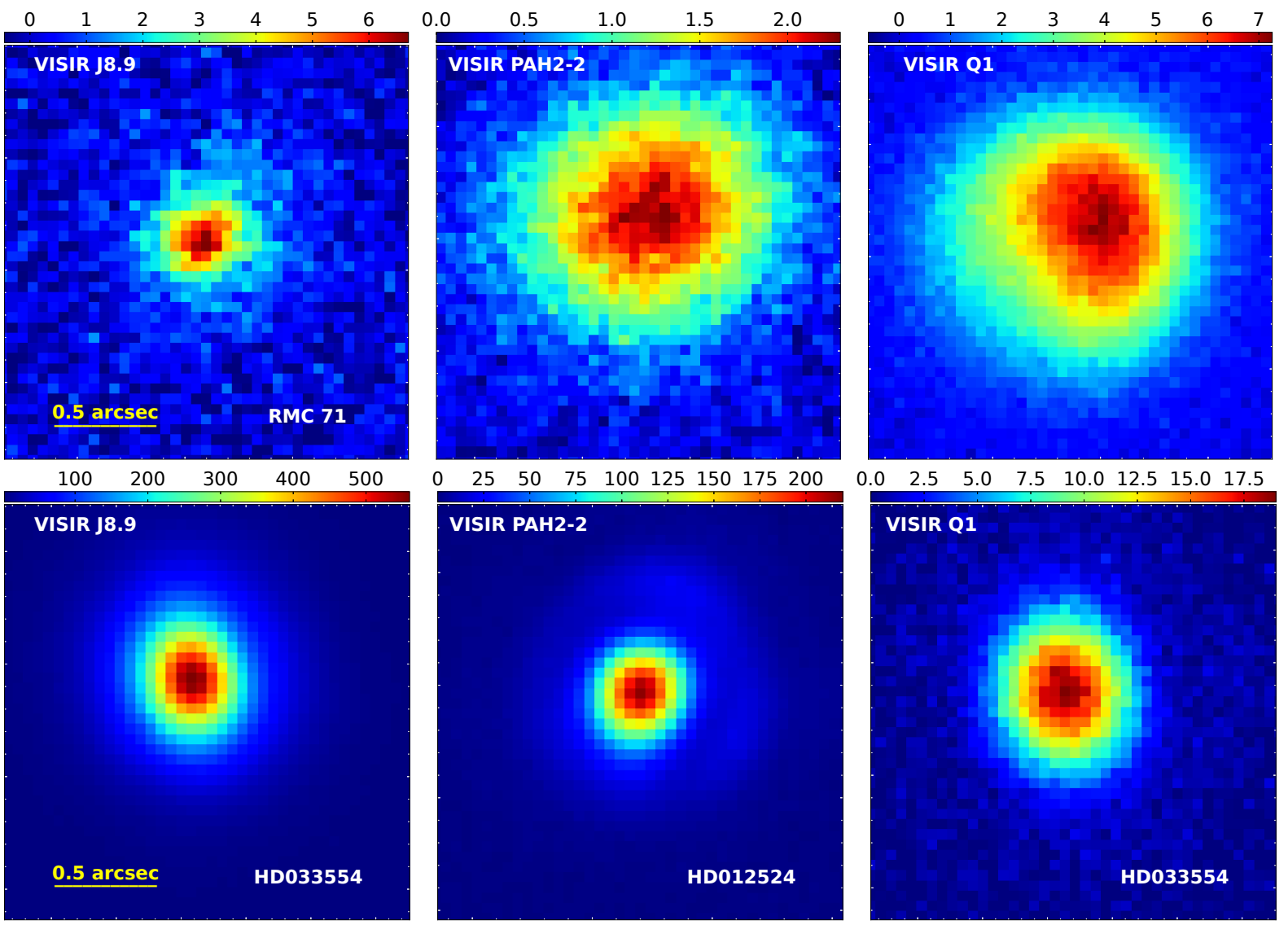

Fig. 3: VISIR images of RMC71 (top panels), and the standard calibrators observed on the same nights (bottom panels, with identifications given in the lower right corners). North is up and east is left. The colour-scale is in arbitrary units.

(Crawford et al. 2016) for the stacking analysis in Sect. 5.2 None of the sources is detected in these maps obtained with the large beam of $1.5^{\prime}$.

Finally, we used the available Infrared Spectrograph (IRS; Houck et al. 2004) spectra from the Combined Atlas of Sources with Spitzer IRS Spectra (CASSIS; Lebouteiller et al. 2011, 2015) or from the IRSA data archive (as SSC Enhanced Products). RMC 127, LHA 120-S 61, and RMC 84 were observed under Program Name/Id: STELLARATLAS/485 and published by Ardila et al. (2010). The RMC66, HD 38489, HD 34665, and LHA 115-S 18 spectra were published in Kastner et al. (2010), although we found that the spectrum of LHA 115-S 18 is not on source. The spectra of RMC 14 and RMC 40 were discussed in Ruffle et al. (2015), and those of RMC 110 and S Dor in Jones et al. (2017). Data of RMC 99 and Sk -69279 are available in the archive: we are not aware of any previous publications. The IRS spectra are shown with grey continuous lines in the flux density distribution plots in Appendix B RMC71 and RMC 66 also have Infrared Space Observatory (ISO) data 3 and low resolution Multiband Imaging Photometer for Spitzer (MIPS; Rieke et al. 2004) SED-mode spectra (presented in Kemper et al. 2010, van Loon et al. 2010, which also include RMC 126) but they are not considered in this work. The IRS spectra are mainly used to guide the interpretation of the infrared photometry when no measurement at $24 \mu \mathrm{m}$ is possible due to confusion.

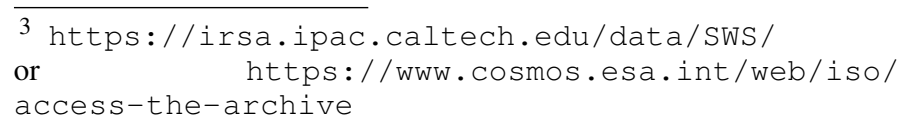

\section{Data analysis}

\subsection{Photometry from Spitzer MIPS24/MIPS70}

A small number of sources are not included in the Spitzer SAGE catalogues, or they have poor catalogue photometry, most likely because of the strict requirements adopted to identify pointsources (Meixner et al. 2006). Indeed we find that these sources are usually extended or embedded in a confused region, requiring a manual extraction of the flux measurement. The most remarkable case is Sk-69279, which appears as a well detected extended bubble in the MIPS24 map, but has no catalogue photometry. We performed aperture photometry for all sources that were either lacking measurements or are extended in the images. As a cross-check, we also measured all targets with catalogue values. Following this verification, we chose to use the existing catalogue photometry for the non-extended sources, unless indicated otherwise.

Our aperture photometry was performed with the astropy package "photoutils". The aperture radii, sky annuli, and aperture corrections, as recommended in the MIPS instrument handbook $\sqrt{4}$, are listed in Tables 3 and 4 , together with the measured flux densities, comparison with the catalogue value when available, and relevant comments on the sources and their environments. The reported flux density errors only account for the uncertainty in determining the background (defined as the standard deviation in the background annulus integrated over the aper-

$4 \longdiv { \text { https://irsa.ipac.caltech.edu/data/SPITZER/ } }$
docs/mips/mipsinstrumenthandbook/50/

Article number, page 6 of 37 
Table 3: MIPS-24 aperture photometry.

\begin{tabular}{|c|c|c|c|c|c|c|c|}
\hline ID & Source & $\begin{array}{l}\text { Ap. radius } \\
\left({ }^{\prime \prime}\right)\end{array}$ & $\begin{array}{l}\text { Sky annulus } \\
\left({ }^{\prime \prime}\right)\end{array}$ & Ap. corr. & $\begin{array}{l}\text { Measured } F_{v} \\
(\mathrm{mJy})\end{array}$ & $\underset{(\mathrm{mJy})}{\text { Catalogue }} F_{v}$ & Comment \\
\hline 1 & RMC 143 & - & - & - & $482.2 \pm 96.4$ & - & from Agliozzo et al. (2019) \\
\hline 2 & RMC 127 & 20 & $20-32$ & 1.15 & $1255 \pm 10$ & $1180 \pm 60$ & \\
\hline 3 & S Doradus & 20 & $20-32$ & 1.15 & $848 \pm 90$ & $898 \pm 22$ & \\
\hline 4 & RMC 110 & 7 & $7-13$ & 2.05 & $\mathbf{1 0} \pm \mathbf{2}$ & $7.39 \pm 0.14$ & $\begin{array}{l}\text { small aperture due to } \\
\text { confusion }\end{array}$ \\
\hline 5 & HD 269582 & 7 & $7-13$ & 2.05 & $7.7 \pm 0.4$ & $7.783 \pm 0.225$ & \\
\hline 6 & RMC 116 & 7 & $7-13$ & 2.05 & $1.4 \pm 0.8$ & $1.293 \pm 0.155$ & marginally detected \\
\hline 7 & HD 269216 & 20 & $20-32$ & 1.15 & $21 \pm 5$ & $6.952 \pm 0.134$ & marginally resolved \\
\hline 8 & RMC 71 & 一 & - & - & - & $>4100 \pm 410$ & saturated \\
\hline 9 & RMC 85 & - & - & - & - & - & not detected/confusion \\
\hline 10 & RMC 123 & 7 & $7-13$ & 2.05 & $15.0 \pm 1.2$ & $16.13 \pm 0.28$ & $\begin{array}{l}\text { small aperture due to } \\
\text { confusion }\end{array}$ \\
\hline 11 & RMC99 & 一 & - & - & - & $49.4 \pm 0.5$ & not detected/confusion \\
\hline 12 & Sk -69279 & 20 & $20-32$ & 1.15 & $22 \pm 5$ & - & bubble \\
\hline 13 & LHA 120-S 119 & 35 & $40-50$ & 1.08 & $703 \pm 23$ & $537.1 \pm 5.3$ & extended \\
\hline 14 & LHA 120-S 61 & 20 & $20-32$ & 1.15 & $1474 \pm 10$ & $1470 \pm 74$ & extended \\
\hline 15 & RMC 74 & 7 & $7-13$ & 2.05 & $3.5 \pm 0.3$ & $3.074 \pm 0.145$ & \\
\hline 16 & RMC 78 & 20 & $20-32$ & 1.15 & $59 \pm 19$ & - & double source \\
\hline 17 & RMC 81 & 35 & $40-50$ & 1.08 & $108 \pm 29$ & - & extended \\
\hline 18 & LHA 120-S 18 & 7 & $7-13$ & 2.05 & $11.5 \pm 1.5$ & $7.882 \pm 0.101$ & $\begin{array}{l}\text { smaller aperture } \\
\text { due to confusion, } \\
\text { lower limit? }\end{array}$ \\
\hline 19 & RMC66 & 20 & $20-32$ & 1.15 & $856 \pm 8$ & $813 \pm 6$ & \\
\hline 20 & RMC 84 & 20 & $20-32$ & 1.15 & $104 \pm 4$ & $99.3 \pm 0.9$ & \\
\hline 21 & HD 34664 & 7 & $7-13$ & 2.05 & $389 \pm 19$ & $411 \pm 3$ & $\begin{array}{l}\text { point source in the bright } \\
\text { ring of another star }\end{array}$ \\
\hline 22 & HD 38489 & 20 & $20-32$ & 1.15 & $490 \pm 42$ & $498 \pm 4$ & \\
\hline 23 & RMC 126 & 20 & $20-32$ & 1.15 & $1098 \pm 15$ & $1120 \pm 10$ & \\
\hline 24 & Sk-69271 & 20 & $20-32$ & 1.15 & $<23$ & - & \\
\hline 25 & RMC 128 & - & - & - & - & - & $\begin{array}{l}\text { not detected } \\
\text { in the vicinity of LBV [2] }\end{array}$ \\
\hline 26 & RMC 149 & - & - & - & - & - & confusion \\
\hline 27 & HD 269604 & 7 & $7-13$ & 2.05 & $\mathbf{0 . 6} \pm \mathbf{0 . 2}$ & 0.737 & \\
\hline 28 & RMC 14 & - & - & - & - & $1.634 \pm 0.131$ & \\
\hline 29 & RMC 40 & 20 & $20-32$ & 1.15 & $29.4 \pm 1$ & $27.0 \pm 0.4$ & \\
\hline 30 & RMC 4 & 20 & $20-32$ & 1.15 & $67.3 \pm 8.8$ & $45.4 \pm 0.5$ & \\
\hline 31 & LHA $115-S 18$ & 20 & $20-32$ & 1.15 & $82.1 \pm 2.6$ & $87.0 \pm 0.6$ & \\
\hline
\end{tabular}

Notes. The numbers highlighted in bold are new measurements with no counterpart in the catalogue or with revised photometry, which are adopted in the analysis.

ture), which generally dominates over the absolute flux calibration error and statistical noise in the source aperture. For nondetected sources, we provide $3 \sigma$ upper limits.

We checked the quality of our photometry against the catalogues (see Tables 3 and 4): the results are consistent with the published values. In bold we highlight our measurements that are used in the later analysis instead of the catalogue values.

\subsection{Photometry and upper limits from Herschel}

Only a few sources were well detected with the Photodetector Array Camera and Spectrometer (PACS, Poglitsch et al. 2010) at 100 and $160 \mu \mathrm{m}$, and their flux densities were taken from the HERITAGE catalogue (Meixner et al. 2013). Additionally, we report in Table 5 new PACS100 photometry for LHA 120-S 119 and HD 38489 (although the latter is only a marginal detection). To maximise the signal-to-noise ratio, we adopted a procedure equivalent to Gaussian fitting. Local peaks were searched for in boxes with size equivalent to the beam. The regions used to evaluate the sky contribution were separated by one or two beams. The $1 \sigma$ total uncertainty is determined by summing in quadrature the standard deviation of the background and the flux calibration uncertainty of $10 \%$. Because of contamination of S Dor from a strong nearby nebular region, we reduced the background spacing to one beam. Nevertheless, the source was not unambiguously detected. For the sources with no detection (either in this work or in the catalogue), we report the $3 \sigma$ upper limit at $100 \mu \mathrm{m}$ determined as described above.

Finally, in PACS160 we only find a single new detection, LHA 120-S 61, which has a flux density of $60 \pm 16 \mathrm{mJy}$. We do not report any new detection obtained at longer wavelengths with the Spectral and Photometric Imaging Receiver (SPIRE) instrument (Griffin et al. 2010). The upper limits at $160 \mu \mathrm{m}$ and longer wavelengths are not provided as they do not add useful information owing to the larger beam sizes and high interstellar dust brightness at such wavelengths. 
Table 4: MIPS-70 aperture photometry and $3 \sigma$ upper limits.

\begin{tabular}{|c|c|c|c|c|c|c|c|}
\hline ID & Source & $\begin{array}{l}\text { Aperture radius } \\
\qquad\left({ }^{\prime \prime}\right)\end{array}$ & $\begin{array}{l}\text { Sky annulus } \\
\left({ }^{\prime \prime}\right)\end{array}$ & Ap. corr. & $\begin{array}{c}\text { Measured } F_{V} \\
(\mathrm{mJy})\end{array}$ & $\underset{(\mathrm{mJy})}{\text { Catalogue }} F_{v}$ & Comment \\
\hline 1 & RMC 143 & 16 & $18-39$ & 2.07 & $<14551$ & - & confusion \\
\hline 2 & RMC 127 & 16 & $18-39$ & 2.07 & $1021.3 \pm 54.4$ & $987 \pm 27$ & \\
\hline 3 & SDoradus & 16 & $18-39$ & 2.07 & $<2090$ & - & confusion \\
\hline 4 & RMC 110 & 16 & $18-39$ & 2.07 & $<1243$ & - & confusion \\
\hline 5 & HD 269582 & 16 & $18-39$ & 2.07 & $<155$ & - & confusion \\
\hline 6 & RMC 116 & 16 & $18-39$ & 2.07 & $<663$ & - & confusion \\
\hline 7 & HD 269216 & 16 & $18-39$ & 2.07 & $128 \pm 71$ & - & $\begin{array}{l}\text { detected } \\
\text { but confused }\end{array}$ \\
\hline 8 & RMC 71 & 16 & $18-39$ & 2.07 & $2029.4 \pm 49.6$ & $1858 \pm 24$ & \\
\hline 9 & RMC 85 & 16 & $18-39$ & 2.07 & $<3864$ & - & confusion \\
\hline 10 & RMC 123 & 16 & $18-39$ & 2.07 & $<477$ & - & confusion \\
\hline 11 & RMC99 & 16 & $18-39$ & 2.07 & $<867$ & - & confusion \\
\hline 12 & Sk -69279 & 16 & $18-39$ & 2.07 & $<214$ & - & confusion \\
\hline 13 & LHA 120-S 119 & 16 & $18-39$ & 2.07 & $447.2 \pm 51.4$ & $428.3 \pm 15.1$ & $\begin{array}{l}\text { marginally } \\
\text { resolved }\end{array}$ \\
\hline 14 & LHA 120-S 61 & 16 & $18-39$ & 2.07 & $635.2 \pm 22.1$ & $579.3 \pm 8.8$ & \\
\hline 15 & RMC 74 & 16 & $18-39$ & 2.07 & $<58$ & - & noise \\
\hline 16 & RMC 78 & 16 & $18-39$ & 2.07 & $<350$ & - & confusion \\
\hline 17 & RMC 81 & 16 & $18-39$ & 2.07 & $150 \pm 56$ & - & $\begin{array}{l}\text { possible detection } \\
\text { but confused }\end{array}$ \\
\hline 18 & LHA 120-S 18 & 16 & $18-39$ & 2.07 & $<330$ & - & confusion \\
\hline 19 & RMC 66 & 35 & $39-65$ & 1.24 & $531.5 \pm 56.2$ & $528 \pm 9$ & \\
\hline 20 & RMC 84 & 16 & $18-39$ & 2.07 & $104 \pm 29$ & - & $\begin{array}{l}\text { possible detection } \\
\text { but confused }\end{array}$ \\
\hline 21 & HD 34664 & 16 & $18-39$ & 2.07 & $<4484$ & 一 & $\begin{array}{l}\text { contaminating } \\
\text { nearby source }\end{array}$ \\
\hline 22 & HD 38489 & 16 & $18-39$ & 2.07 & $<769$ & - & confusion \\
\hline 23 & RMC 126 & 16 & $18-39$ & 2.07 & $299 \pm 126$ & $380 \pm 24$ & $\begin{array}{l}\text { inhomougeneous } \\
\text { background }\end{array}$ \\
\hline 24 & $\mathrm{Sk}-69271$ & 16 & $18-39$ & 2.07 & $<298$ & - & confusion \\
\hline 25 & RMC 128 & 16 & $18-39$ & 2.07 & $<218$ & 一 & $\begin{array}{l}\text { contaminating } \\
\text { nearby source }\end{array}$ \\
\hline 26 & RMC 149 & 16 & $18-39$ & 2.07 & $<16700$ & - & confusion \\
\hline 27 & HD 269604 & 16 & $18-39$ & 2.07 & $<53$ & 一 & marginal detection \\
\hline 28 & RMC 14 & 16 & $18-39$ & 2.07 & $<1612$ & - & confusion \\
\hline 29 & RMC 40 & 16 & $18-39$ & 2.07 & $58.7 \pm 8.0$ & - & \\
\hline 30 & RMC 4 & 16 & $18-39$ & 2.07 & $<338$ & - & confusion \\
\hline 31 & LHA 115-S 18 & 16 & $18-39$ & 2.07 & $<120$ & 一 & confusion \\
\hline
\end{tabular}

Notes. The numbers highlighted in bold are new measurements with no counterpart in the catalogue or with revised photometry, which are adopted in the analysis.

\section{The LBV SED classification scheme and colour-magnitude diagram}

We first need to ensure to limit our sample to LBV stars and find a classification criterion that does not rely on the spectroscopic and photometric variations only, but also on properties of the stellar ejecta. Table 1 summarises the relevant information on our sample, including the stellar classification from the literature. Stellar parameters, extinction, and nebular size when known are summarised in Table A.1 of Appendix A. Unfortunately, we cannot provide information on the binary status, as to our knowledge this is known only for RMC 14 in the SMC and RMC 81 in the LMC. The complete set of photometry from this work and from the catalogues are in Tables C.1, C.2 and C.3 of Appendix C For all sources, the flux density distribution starting from $1 \mu \mathrm{m}$ are shown in Figs. B.1, B.2, B.3, B.4, B.5, B.6 of Appendix $\mathrm{B}$, where in addition to the photometric points the stellar continuum (on the basis of stellar parameters as in Table A.1p and the IRS spectra are also included when available.

In their sample of 1750 massive stars in the LMC, Bonanos et al. (2009) included six LBVs (S Dor, RMC 127, RMC 71, RMC 110, RMC 85, HD 269582) and concluded that these stars do not represent a homogeneous class of objects on the basis of their mid-infrared colours. We identify four classes based on the individual infrared spectral energy distributions (SED):

1. Sources with a dusty nebula peaking in the mid- to farinfrared (Class 1). These can be further divided into two groups: with or without a significant free-free excess above the stellar photosphere (Classes 1a and 1b, respectively). An example can be found in the top-right panel in Fig. 4 where the object shows a moderate free-free continuum up to $10 \mu \mathrm{m}$, and at longer wavelengths a dominant infrared excess due to dust in the extended nebula, previously imaged by Weis et al. (2003) and Agliozzo et al. (2012). By con- 
Table 5: PACS-100 point source extraction and $3 \sigma$ upper limits.

\begin{tabular}{|c|c|c|c|c|}
\hline ID & Source & $\underset{(\mathrm{mJy})}{\text { Measured } F_{v}}$ & $\underset{(\mathrm{mJy})}{\text { Catalogue }} F_{V}$ & Comment \\
\hline 1 & RMC 143 & - & $636 \pm 254$ & \\
\hline 2 & RMC 127 & $535 \pm 65$ & $493 \pm 43$ & \\
\hline 3 & SDoradus & $<1100$ & - & $\begin{array}{c}\text { not detected } \\
\text { due to confusion }\end{array}$ \\
\hline 4 & RMC 110 & $<350$ & - & \\
\hline 5 & HD 269582 & $<270$ & 一 & \\
\hline 6 & RMC 116 & $<560$ & 一 & \\
\hline 7 & HD 269216 & $<283$ & - & \\
\hline 8 & RMC 71 & $669.0 \pm 68.5$ & $706 \pm 48$ & \\
\hline 9 & RMC 85 & $<1850$ & - & \\
\hline 10 & RMC 123 & $<206$ & - & \\
\hline 11 & RMC99 & $<699$ & 一 & \\
\hline 12 & SK-69279 & $<137$ & 一 & \\
\hline 13 & LHA 120-S 119 & $204.7 \pm 33.6$ & - & marginally resolved \\
\hline 14 & LHA 120-S 61 & $292.8 \pm 37.6$ & $305 \pm 31$ & \\
\hline 15 & RMC 74 & $<38$ & - & \\
\hline 16 & RMC 78 & $<195$ & - & \\
\hline 17 & RMC 81 & $<105$ & 一 & \\
\hline 18 & LHA 120-S 18 & $<132$ & - & \\
\hline 19 & RMC 66 & $318 \pm 33$ & $320 \pm 26$ & \\
\hline 20 & RMC 84 & $<59$ & - & \\
\hline 21 & HD 34664 & $<1805$ & 一 & \\
\hline 22 & HD 38489 & $123.3 \pm 90.8$ & 一 & $\begin{array}{l}\text { marginal detection } \\
\text { due to confusion }\end{array}$ \\
\hline 23 & RMC 126 & $199 \pm 52$ & $136 \pm 29$ & \\
\hline 24 & Sk -69271 & $<151$ & - & \\
\hline 25 & RMC 128 & $<94$ & - & \\
\hline 26 & RMC 149 & $<34800$ & - & \\
\hline 27 & HD 269604 & $<37$ & 一 & \\
\hline 28 & RMC 14 & $<440$ & - & \\
\hline 29 & RMC 40 & $<40$ & - & \\
\hline 30 & RMC 4 & $<434$ & - & \\
\hline 31 & LHA 115-S 18 & $<62$ & - & \\
\hline
\end{tabular}

Notes. The numbers highlighted in bold are new measurements with no counterpart in the catalogue or with revised photometry, which are adopted in the analysis.

trast, the top left panel shows an example of strong free-free emission.

2. Sources with only a free-free emission excess (Class 2). An example is given in the middle-left panel in Fig. 4 . For these sources the MIPS24 flux density is consistent with a power law describing the free-free continuum.

3. Sources dominated at all wavelengths $\gtrsim 2 \mu \mathrm{m}$ by a hot dusty component, suggestive of a disc close to the star (Class 3 ). In the literature, these are all classified as $\mathrm{B}[\mathrm{e}]$ supergiants (see Table A.1). An example of a Class 3 SED is HD 34664, shown in the second-right panel of Fig. 4.

4. Featureless sources with a single component consistent with the stellar photosphere at all wavelengths (Class 4). An example is presented in the bottom panel of Fig. 4

Table 1 summarises the classification of the sources in the scheme proposed here. Among them, only one object cannot be classified, RMC 85. This is an active LBV (van Genderen et al. 1998). The star is embedded in a very crowded region. Because of the absence of reliable photometry longward of $\sim 20 \mu \mathrm{m}$ (confirming Bonanos et al. 2009), we are not able to distinguish whether it is SED Class 1 or Class 2 (see Fig. B.4).
The dust composition of sources in Class 1 typically comprises amorphous silicates, although a mixed chemistry is sometimes reported, like in the case of RMC 71 in the LMC (Guha Niyogi et al. 2014) or HD 168625 in the Milky Way (Umana et al. 2010). Crystalline silicates are also found in LBVs, albeit rarely (e.g. RMC 71 and HR Car, Voors et al. 1999; Umana et al. 2009 respectively), while they seem to be a common feature of sgB[e] stars (e.g. Kastner et al. 2006, 2010, de Wit et al. 2014).

In Fig. 5 we present the [8.0] versus [8.0]-[24] colourmagnitude diagram (CMD) of stars in the LMC, depicting the position of the LBV sample analysed in this work (filled circles of various colours representing the different LBV SED classes as described in the legend); early and late supergiants as in Bonanos et al. (2009) (downward pointing triangle symbols); grey diamonds are low- and intermediate-mass, but also some high mass stars, planetary nebulae and SN remnants from Jones et al. (2015); AGBs and RSGs from Riebel et al. (2012) and Young Stellar Objects (YSOs) from Whitney et al. (2008). Stars with SED Classes 2, 3 and 4 occupy the same region as RSGs and other types of massive stars in the diagram, which Bonanos et al. found indistinguishable from AGBs and young stellar objects, 

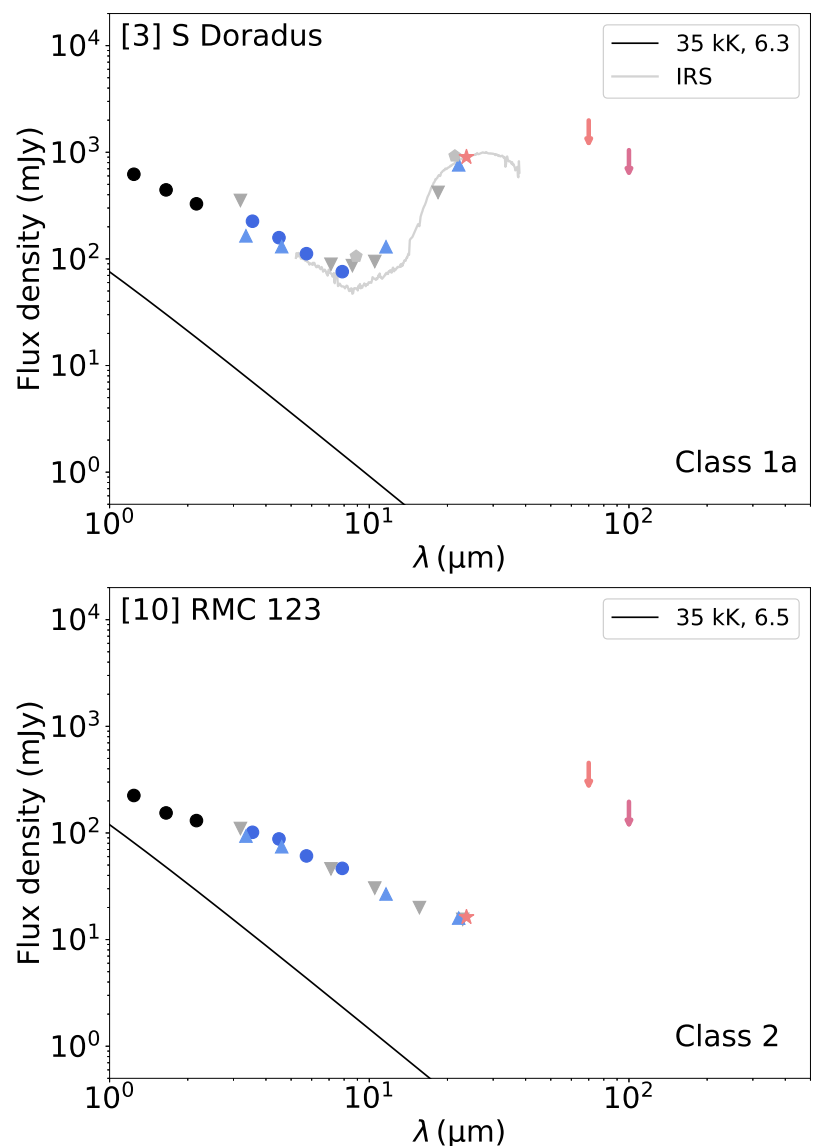
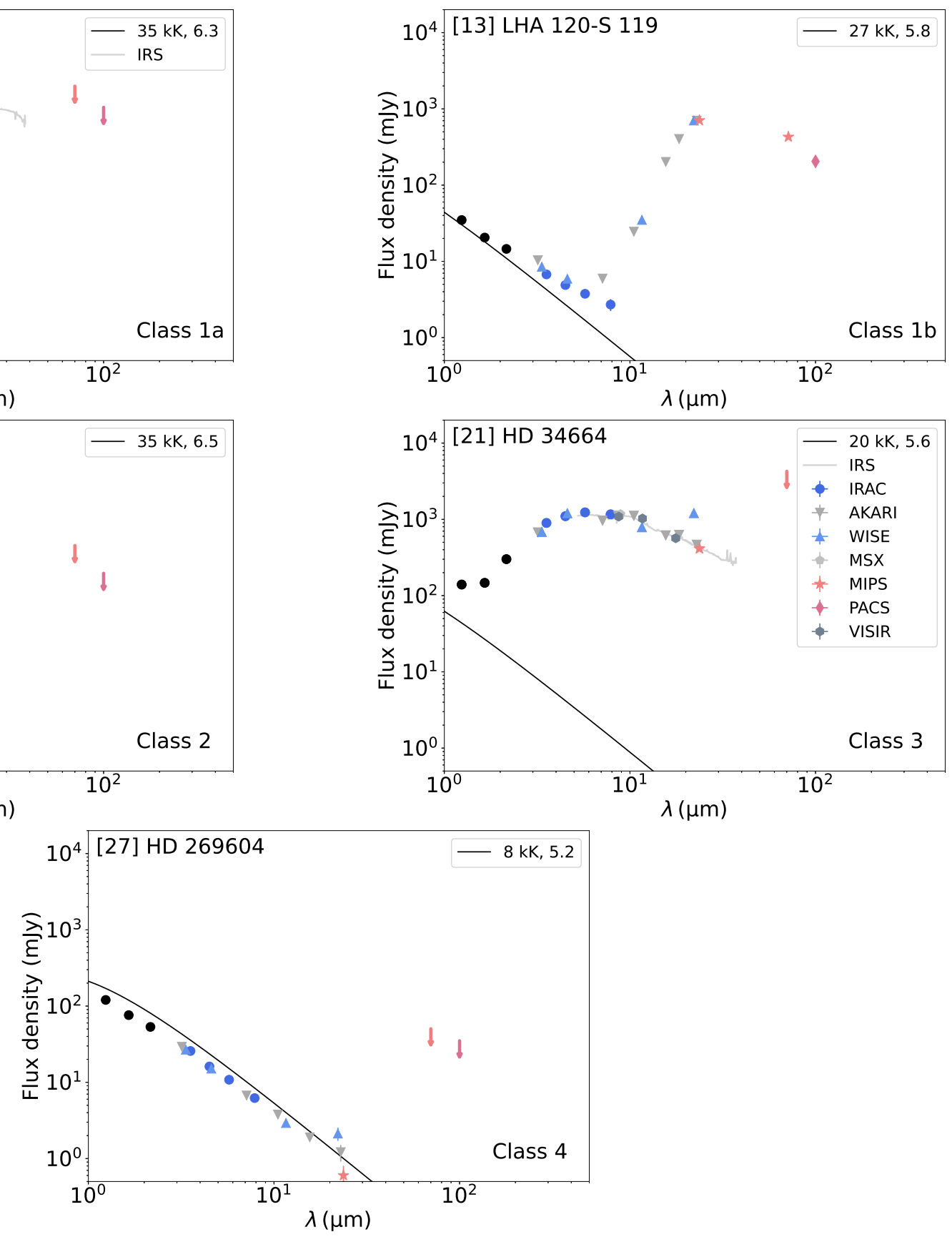

Fig. 4: Examples of each of the four LBV SED classes defined in Sect. 4

highlighting the importance of using wide-baseline photometry. However, we notice some differences: Class 2 objects tend to be fainter than RSGs at $8 \mu \mathrm{m}$, due to lacking a hot dusty envelope; Class 3 are usually brighter than RSGs, due to their hot dusty disc; the only Class 4 object with MIPS24 photometry is the faintest in this region of the diagram, consistent with no excess above the photosphere. For stars in the SED Class 1, the [8.0]-[24] colours coincide with the sample of stars in Jones et al. However, the nature of 7 out of their 35 objects was not spectroscopically confirmed. These could all be sources with a dusty gaseous nebula (e.g. SN remnants, planetary nebulae, LBV nebulae). For stars in the SMC we refer to the colour-diagram by Bonanos et al. (2010), who included all the LBVs in the census (Richardson \& Mehner 2018). Bonanos et al. found that LBVs are among the most luminous stars at $24 \mu \mathrm{m}$, but they are not clearly separated from other types of star.

\subsection{Class 1: sources with dusty nebulae peaking in the mid- to far-infrared}

This class contains well-known extended MCs LBV nebulae (LBVNe), extensively studied in the literature. Some of them are associated with very active LBVs (Walborn et al. 2017). Some stars are classified as candidate LBVs: these are stars that do not satisfy all LBV classification criteria (both observed spectroscopic and photometric variability; Humphreys \& Davidson 1994), however the presence of a circumstellar nebula around some of them suggests that they are ex/dormant LBVs. 


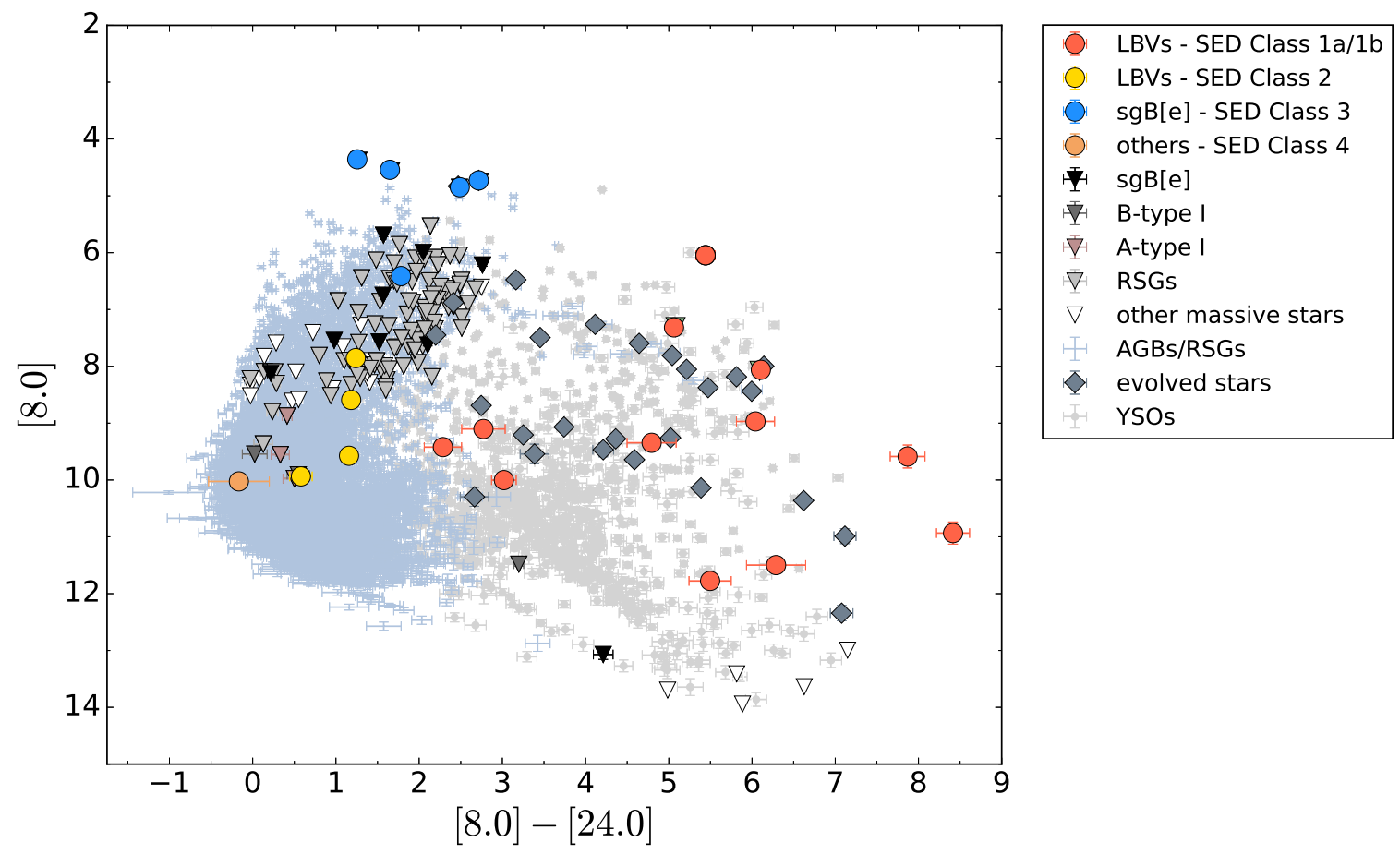

Fig. 5: Mid-infrared colour-magnitude diagram for stars in the LMC. The LBVs are shown with filled circles in different colours, depending on the SED class defined in this work. Several types of supergiant stars (RSGs, sgB[e], supergiants A and B, six LBVs and other massive stars) as in Bonanos et al. (2009) are displayed with triangles of different colours as identified in the legend. Dusty evolved stars from Jones et al. (2015) are shown with diamond symbols (these include post-AGBs, planetary nebulae, sgB[e] stars, one LBV, one WR and two SN remnants). Pixel symbols indicate the AGBs and RSGs from Riebel et al. (2012) and point symbols are confirmed and candidate YSOs from Whitney et al. (2008).

Depending on whether or not they have an excess of nearinfrared emission due to the ionised stellar wind, stars in this class can be further divided into: Class 1a, which are objects that present free-free emission from the wind; Class $1 \mathrm{~b}$, which are objects with no detected free-free emission from the wind. For two objects, Sk-69279 and RMC 78, we report the first identification of a dusty circumstellar nebula. The dust component in these sources peaks between 24 and $100 \mu \mathrm{m}$, similarly to several Galactic objects with extended nebulae (typically with sizes of the order of $0.1-1$ pc, e.g. G79.29+0.46, Pistol Star, AG Car, AFGL2298, HR Car; Agliozzo et al. 2014; Lau et al.|2014; Vamvatira-Nakou et al.|2015; Buemi et al.2010, 2017, and references therein). Among the sources in this class, five (RMC 127, RMC 71, S Dor, LHA 120-S 61, and RMC 40) have an IRS spectrum and all of them show, together with the thermal continuum, the silicate bump around $10-12 \mu \mathrm{m}$.

The objects classified as belonging to Class 1 are briefly introduced (see Fig. B.1 and B.2 in the Appendix):

S Dor is the prototype of the most common LBV variability (a summary of the pioneering works can be found in Wolf 1989). The infrared excess from a dusty nebula was reported in Bonanos et al. (2009) from mid-infrared photometry and in Jones et al. (2017) from the IRS spectrum. The power-law behaviour in the IRS data below $10 \mu \mathrm{m}$ is more likely consistent with strong free-free emission in the ionised stellar wind rather than the stellar photosphere. The bright surrounding environment does not allow for the disentanglement of the nebula at $70 \mu \mathrm{m}$ and beyond. The SED Class of this object is 1a.

RMC 127: this star belongs to the group of active LBVs (Walborn et al. 2008, 2017). This is another object with SED Class 1a. See Agliozzo et al. (2017b) for a multiwavelength study of its nebula.

LHA 120-S 61: candidate LBV (Humphreys \& Davidson 1994) with SED Class 1a, its dusty nebula was modelled by Agliozzo et al. (2017a).

Sk -69279: candidate LBV with SED Class 1a, the nebula was previously imaged by $H S T$ (Weis \& Duschl 2002). We find that at $24 \mu \mathrm{m}$ it appears as a bubble, resembling the morphology revealed in the optical. To our knowledge, this is the first report of dust in this object.

LHA 120-S 119: candidate LBV (van Genderen 2001) with a known nebula (SED Class 1a) that was previously imaged at different wavelengths (Agliozzo et al. 2012, and references therein). In the infrared it is possible to notice a thermal component of dust, detected up to $100 \mu \mathrm{m}$. The nebula is marginally resolved at $24 \mu \mathrm{m}$, revealing a marked asymmetry similar to the optical and radio images. This asymmetry was explained as a bow-shock, given the run-away nature of its associated star (Danforth \& Chu 2001).

RMC 81: infrared emission from cool dust around this candidate LBV (van Genderen 2001) was first reportedly detected by IRAS at $60 \mu \mathrm{m}$ (Beichman et al. 1988; Tubbesing et al. 2002). However, inspection of the MIPS70 image, comparison with the MIPS photometry, the lack of 25 or $12 \mu \mathrm{m}$ IRAS detections, and considering the $1 \sigma$ position offset, it appears that this flux was greatly elevated by ISM confusion. The object is detected in both MIPS bands, even at $70 \mu \mathrm{m}$ where the surrounding environment is bright. The star is an eclipsing binary (Stahl et al. 1987). The SED Class of this object is 1a.

LHA 120-S 18: at $24 \mu \mathrm{m}$ and beyond, the environment around this candidate LBV (van Genderen 2001) is bright and the object is not distinguishable from the background. The SED Class 
of this object is $1 \mathrm{a}$.

RMC 143: the dusty and massive nebula (SED Class 1b) was extensively studied by Agliozzo et al. (2019), who also confirmed the LBV status of this star.

RMC 71: this star also belongs to the group of very active LBVs (Mehner et al. 2013, Walborn et al. 2017), with SED Class 1b. It is possible to observe variability in the infrared SED (compare the mid-infrared photometry with the IRS spectrum), as the data were acquired at different epochs during the most recent stellar outburst (Mehner et al. 2017). Here we show a similar dataset, but extended to longer wavelengths. The entire SED was previously modelled by Guha Niyogi et al. (2014).

RMC 78: at $24 \mu \mathrm{m}$ the object (a candidate LBV, van Genderen \& Sterken 1999) appears as a double source (SED Class 1b).

RMC 40: this star belongs to the group of confirmed LBVs (Szeifert et al. 1993) in the SMC. The first detection at $24 \mu \mathrm{m}$ was reported by Bonanos et al. (2010). Here we add a new clear detection at $70 \mu \mathrm{m}$. The SED Class of this object is $1 \mathrm{~b}$. An IRS spectrum is available and suggests that the dust emission peaks between the two MIPS bands.

HD 269216: a confirmed LBV (Prinja \& Schild 1991), this object is well detected and marginally resolved in MIPS 24 . We add a new measurement as the catalogue PSF fitting may have missed a large fraction of its flux density. The SED Class of this object is $1 \mathrm{~b}$.

RMC 110: this is a confirmed LBV manifesting the eruptive phenomenon a few times in the past decades (Stahl et al. 1990; Campagnolo et al. 2018). Because of confusion at wavelengths beyond $\sim 20 \mu \mathrm{m}$ it is not possible to obtain reliable photometry of dust in its ejecta (SED Class 1b).

\subsection{Class 2: sources with free-free excess only}

This class consists of the following objects (see Fig. B.3

RMC 116 is a strongly active S Dor variable (van Genderen 2001). Its SED shows an ionised stellar wind in the form of a free-free excess above the stellar photosphere detected up to $24 \mu \mathrm{m}$.

RMC 123 and RMC 99 belong to a sub-group proposed by Stahl et al. (1984) and Stahl \& Wolf (1987), characterised by an excess of free-free emission from a gaseous disc, rich in forbidden lines, but with no dust features. The free-free emission of RMC 123 is detected up to $24 \mu \mathrm{m}$. At longer wavelengths, the SED of RMC 99 is dominated by contamination from neighbouring sources so that the presence of an infrared excess is questionable. Both stars are catalogued as weakly active S Dor variables in van Genderen (2001) and are considered candidate LBVs. RMC 99 was observed with IRS, but the spectrum is likely dominated by interstellar gas and dust.

HD 269582 is a very active LBV (Walborn et al. 2017) with a strong ionised stellar wind detected up to $24 \mu \mathrm{m}$. Differences between 2MASS and IRAC data suggest that the star was active (highly variable) in the years of the infrared observations.

RMC 74 is a candidate LBV (van Genderen 2001) with a SED similar to that of RMC 116.

RMC 14, a well-known multiple-system containing an active LBV in the SMC (e.g. Barba et al. 1996), shows an SED similar to HD 269582, with a strong free-free excess above the continuum (a similar SED can also be found in Bonanos et al. 2010). This is also seen in two other active LBVs (RMC 127, Agliozzo et al. 2017a, and S Dor). The system was observed with IRS, but, because of the large slit width, the spectrum is likely dominated by interstellar gas and dust.

\subsection{Class 3: sources dominated by a hot dusty disc}

RMC 66, RMC 84, HD 38489, HD 34664, RMC 126, RMC 4, and LHA 115-S 18: These stars are all known in the literature as supergiant B[e] stars (e.g. Zickgraf et al. 1996b; van Genderen \& Sterken 1999; Aret et al. 2012, Bonanos et al. 2009, 2010, Kastner et al. 2006, 2010), but sometimes they are also listed in the sample of candidate LBVs because of other observational commonalities (e.g. variability of emission lines and P Cyg profiles, van Genderen 2001). Their SEDs are suggestive of a different dust formation mechanism. Dust in these stars forms in the equatorial plane (perhaps as a consequence of fast rotation or binarity), and resides in a circumstellar disc-like configuration, resembling the mid-infrared spectra of $\mathrm{T}$ Tauri and disc-enshrouded Herbig Ae/Be stars (Kastner et al. 2006, 2010). Furthermore, observations suggest the silicates to be crystalline, which requires long-lived dust particles permanently close to the star, as opposed to outflowing material like in the case of LBV eruptions (for a review, see de Wit et al. 2014). The IRS spectrum, and IRAC and MIPS images of HD 38489, RMC 66, LHA 115-S 18, RMC 126 and HD 34664 first appeared in Kastner et al. (2006, 2010).

\subsection{Class 4: sources with no evident free-free or dust excess}

This class comprises the following objects:

HD 269604 was classified as A1 Ia-0 by Osmer (1973). It appears in several works as a candidate LBV, but van Genderen (2001) flagged it as non-candidate. We do not find evidence of free-free and/or dust infrared excess.

RMC 128 (B2 Ia with N weak, Fitzpatrick 1991) was identified as non-candidate by van Genderen, but it is mentioned as a candidate LBV in several other works. RMC 128 is only $20^{\prime \prime}$ away from RMC 127, which is much more luminous and the star is hard to discern in the mid-infrared. In the near-infrared, there is no evidence of free-free and dust excess. We do not find any observational argument to keep the star in the list of candidate LBVs, although its spatial proximity to the very active RMC 127 certainly makes for an interesting target (e.g. if the two stars are co-eval).

RMC 149 was characterised by van Genderen (2001) as a weakly active S Dor variable (with low-amplitude variations over decades). However, Fariña et al. (2009) re-classified it as O8.5 II((f)). We do not find typical signatures of LBV nebular features (ionised gas and/or dust excess). The infrared images reveal that the source is embedded in a crowded environment.

Sk-69271 is a yellow supergiant (YSG) (Neugent et al. 2012). It appeared in the list of candidate LBVs because of nearby nebular material resembling a half shell, most likely of interstellar origin (Weis et al. 1997). This source is particularly faint already at $8 \mu \mathrm{m}$ and does not show any features at longer wavelengths. For lack of other evidence to keep these stars in the LBV sample, we discard them from further analysis and also suggest their removal from the LBV census (the most recent study to mention is Richardson \& Mehner 2018).

\section{Modelling the infrared spectral energy distributions with grey-body fitting}

\subsection{Individual stars}

Several dusty LBV nebulae are extended. Optical instruments detected $\mathrm{H}_{\alpha}$ emission, almost unextinguished by the dust grains 
Table 6: Summary of estimated dust properties.

\begin{tabular}{lcccr}
\hline \hline Source & $\begin{array}{c}\text { Dust Mass } \\
\left(10^{-2} \mathrm{M}_{\odot}\right)\end{array}$ & $\begin{array}{c}\text { Dust } T \\
(\mathrm{~K})\end{array}$ & $\beta$ & Ref. \\
\hline LHA 120-S 119 & $1.2_{-0.7}^{+2.2}$ & $108_{-10}^{+9}$ & $1.03 \pm 0.045$ & this work \\
RMC 127 & $0.2-2$ & $71-90$ & $1.5-2.0$ & {$[1]$} \\
LHA 120-S 61 & $0.5-3$ & $105-145$ & $0.55-1.5$ & {$[2]$} \\
RMC 143 & $5.5 \pm 1.8$ & $62-80$ & $1.0-1.6$ & {$[3]$} \\
RMC 71 & 1 & $107_{-7}^{+10}$ & N/A & {$[4],[5]$} \\
& $0.9_{-0.2}^{+0.3}$ & $100 \pm 10$ & $1.8 \pm 0.2$ & this work \\
\hline HD 269216 & $0.8 \pm 0.7$ & $62 \pm 6$ & 1.3 (fixed) & this work \\
RMC 81 & $0.4 \pm 0.2$ & $81 \pm 8$ & 1.3 (fixed) & this work \\
RMC 40 & $0.31 \pm 0.07$ & $75 \pm 2$ & 1.3 (fixed) & this work \\
\hline
\end{tabular}

References. [1] Agliozzo et al. (2017b); [2] Agliozzo et al. (2017a); [3] Agliozzo et al. (2019); [4] Guha Niyogi et al. (2014); [5] van Loon et al. (2010).

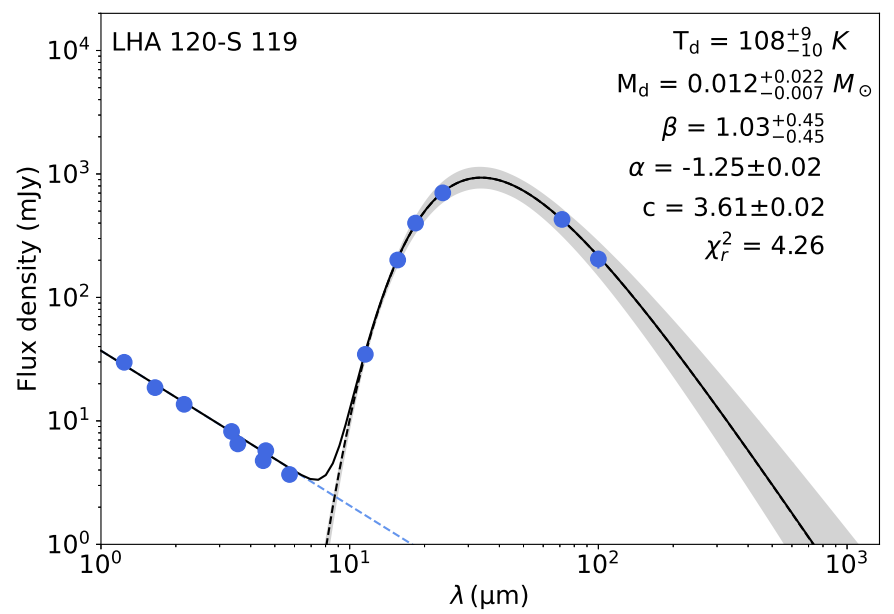

Fig. 6: Example of single grey-body fit of the 2MASS, WISE, Spitzer, and Herschel data, similarly to Agliozzo et al. (2017a b. 2019).

(Agliozzo et al. 2012). Indeed the extinction $\mathrm{A}_{\mathrm{H}_{\alpha}}$ was mapped in three nebulae, revealing relatively low values and thus suggesting an optically thin configuration for the dust (Agliozzo et al. 2017a b, 2019). For such sources we can assume that radiative transfer effects are negligible. Among the stars classified as Class 1 , five have a detection in at least one PACS filter, namely RMC 71, RMC 127, RMC 143, LHA 120-S 61, and LHA 120S 119. With the exception of LHA 120-S 119, the dust mass for these sources has been previously determined in the literature (see Table 6). For LHA 120-S 119, we thus fit the infrared SED with a greybody to derive the characteristic temperature, dust mass and $\beta$ parameter (Fig. 6), using an absorption coefficient value $\kappa_{850 \mu \mathrm{m}}$ of $1.7 \mathrm{~cm}^{2} \mathrm{~g}^{-1}$ (Sodroski et al. 1987), the same as Agliozzo et al. (2017a b, 2019).

We assume a distance of $49.97 \mathrm{kpc}$ for the LMC (Pietrzyński et al. 2013) for all sources. The distances to individual sources will vary around this nominal value due to the inclined geometry and finite thickness of the LMC, but the errors due to this single distance assumption are expected to be less than $2 \mathrm{kpc}$ or 4\% (van der Marel \& Cioni 2001, and considering a maximum separation of any source from the LMC centre of $2^{\circ}$ ). This uncertainty $\left(\sigma_{D} \sim 1 \mathrm{kpc} \sim 2 \%\right)$ is an almost negligible contribution to the dust mass uncertainty compared to the systematic uncertainty in $\kappa$, and the typical measurement uncertainties of the far-infrared photometry.

Radiative transfer modelling was adopted to fit the data of RMC 71 by Guha Niyogi et al. (2014). For comparison, we also fit the data of RMC 71 with a grey-body and find a dust mass which agrees with the literature value. Three other sources (RMC 40, RMC 81, HD 269216) are detected in both the 24 and $70 \mu \mathrm{m}$ MIPS bands but not in the PACS bands. Their measurements suggest that the thermal emission peaks between 24 and $70 \mu \mathrm{m}$. We fit these data by fixing the parameter $\beta=1.3$ (the median of the $\beta$ values determined for the five LMC LBVs above, see also Table 6). The distance assumed for the SMC LBV RMC 40 is $62.44 \mathrm{kpc}$ (Graczyk et al. 2020). We note that the uncertainty of HD 269216 is nearly $100 \%$ due to the marginal detection in MIPS70. For the other Class 1 sources the lack of detection longward of $24 \mu \mathrm{m}$ does not allow to constrain the dust parameters. The results of this analysis are summarised in Table 6

\subsection{Stack analysis of $L B V$ s in the $L M C$}

Given the low detection rates of individual sources at $70 \mu \mathrm{m}$ and longer, largely due to confusion, we have also carried out a stacking analysis to provide a statistical measure of total dust content. The stacking analysis adopted here consists in co-adding the images, at a given wavelength, of individual sources with known coordinates, in order to reduce the noise by averaging, resulting in an aggregate flux density measurement. This technique is particularly useful when the target sources are not detected. The stacking procedure is described in Kurczynski \& Gawiser (2010), and references therein. Here, we do not adopt any deblending method described by the authors, as the confusion in our case is dominated by diffuse ISM emission, of which a-priori positional information is difficult to obtain. For the stacking analysis we considered all confirmed and candidate LBVs (SED Classes 1 and 2), thus excluding $\operatorname{sgB}[\mathrm{e}]$ stars and the sources discarded in Sect. 4 The LMC stack sample thus comprises the first 18 entries in Table 1 . In the SMC there are only two sources to consider (ID number 28 and 29 in the same table), of which only one clearly shows a dust component (number 29). For this reason, we perform the stacking analysis on the LBVs (Class 1 and 2 in Table 1 ) of the LMC only.

To obtain stacked images, for each source we created cutouts of the SAGE and HERITAGE mosaic of the LMC centred, to the nearest pixel, at the sky coordinates as in Table 1, after crosschecking the coordinates with the actual detections in the map. 
Table 7: Flux densities from the stacked data.

\begin{tabular}{|c|c|c|c|c|}
\hline Filter & $\begin{array}{c}\lambda_{\text {eff }} \\
(\mu \mathrm{m})\end{array}$ & LBVs & $\begin{array}{c}\text { LBVs } \\
\text { (w/o RMC143) } \\
\text { (Jy) }\end{array}$ & $\begin{array}{c}\text { x-AGBs } \\
\text { (Jy) }\end{array}$ \\
\hline$J^{a}$ & 1.235 & $3.430 \pm 0.068$ & $3.330 \pm 0.066$ & $5.951 \pm 0.595$ \\
\hline$H^{a}$ & 1.662 & $2.761 \pm 0.070$ & $2.680 \pm 0.068$ & $16.128 \pm 1.613$ \\
\hline$K_{S}^{a}$ & 2.159 & $2.430 \pm 0.050$ & $2.370 \pm 0.050$ & $32.604 \pm 3.260$ \\
\hline IRAC $3.6^{a}$ & 3.550 & $0.776 \pm 0.024$ & $0.735 \pm 0.023$ & $83.965 \pm 8.397$ \\
\hline IRAC4. $5^{a}$ & 4.493 & $0.604 \pm 0.017$ & $0.574 \pm 0.016$ & $93.59 \pm 9.36$ \\
\hline IRAC $5.8^{a}$ & 5.731 & $0.470 \pm 0.013$ & $0.447 \pm 0.012$ & $104.07 \pm 10.42$ \\
\hline IRAC $8.0^{a}$ & 7.872 & $0.541 \pm 0.018$ & $0.500 \pm 0.020$ & $104.18 \pm 10.42$ \\
\hline WISE $^{a}$ & 11.56 & $0.996 \pm 0.082$ & $0.795 \pm 0.080$ & - \\
\hline MIPS24 ${ }^{b}$ & 23.675 & $12.4 \pm 1.7$ & $11.7 \pm 1.5$ & $44.95 \pm 4.64$ \\
\hline MIPS70 ${ }^{b}$ & 71.42 & $4.3 \pm 2.6$ & $4.1 \pm 0.8$ & $7.2 \pm 1.6$ \\
\hline $\operatorname{PACS} 100^{b}$ & 100.0 & $2.34 \pm 0.31$ & $1.66 \pm 0.220$ & $4.1 \pm 1.4$ \\
\hline PACS $160^{b}$ & 160.0 & $0.82 \pm 0.40$ & $>0.41 \pm 0.16^{c}$ & $<4.26^{d}$ \\
\hline SPIRE $250^{b}$ & 250.0 & $>0.122 \pm 0.037^{c}$ & - & - \\
\hline
\end{tabular}

Notes. ${ }^{(a)}$ Summed photometry from catalogues. ${ }^{(b)}$ Photometry of point-source at the centre of stacked images. ${ }^{(c)}$ Lower limit fluxes are measured fluxes from stacks of subsets of the least confused or brightest sources (see text). ${ }^{(d)} 3 \sigma$ statistical upper limit from the noise in the stacked image.

As a Wide-Field Infrared Survey Explorer (WISE) image of the whole LMC galaxy is not available, in this case we queried the WISE image service in the IRSA website and downloaded images centred at the sky coordinates of each source. For none of the instruments it was necessary to re-sample the images as they originate from the same grid. Images of the following bands were stacked: WISE W3, MIPS24, MIPS70, PACS100, PACS160, and SPIRE250. For each band we summed the cutouts of all sources. The resulting stacked images are shown in Fig.7, while the associated photometry is summarised in Table 7, In the same figure, we also show the same stack images without RMC 143, which although the most massive LBV nebula recorded so far in the LMC (Agliozzo et al.2019), is embedded in a very bright and crowded region, just south of the 30 Doradus star forming complex. The comparison of the resulting photometry allows us to assess the relative contribution of the other stars to the infrared emission.

Following the same method, we also stacked cutout images extracted from the LMC $1.4 \mathrm{~mm}$ SPT+Planck map of Crawford et al. (2016). However, we did not obtain any detection above the noise $(\sim 250 \mathrm{mJy} /$ beam $)$ and so do not show the resulting image here.

We then determined the aggregate flux density of the combined source by performing a 2-D Gaussian fitting in each image. Peak height, $x$ and $y$ centre positions and background level are fitted parameters; the width parameters $\sigma_{a}$ and $\sigma_{b}$ were fixed to the instrumental beam $\sigma$ for MIPS70, PACS100, and PACS160 images, while they were left as free parameters (along with a position angle) for the WISE W3 and MIPS24 fitting because at these wavelengths sources were slightly resolved. We used the non-linear least-squares method of the Scipy Optimize package (Virtanen et al. 2020) for minimising the fitting function residuals. To account for systematic errors in this procedure (e.g. missing flux in sidelobes), we corrected the values by a factor determined in each filter by comparing our own estimates of individual test sources with catalogue measurements. Figure 7 shows the stacked images and their residuals after subtraction of the 2-D Gaussian. We do not include SPIRE 250 because there is no source detected, owing to source confusion over the large beam. For the stack analysis at the IRAC bands, we only summed the photometry from the catalogue, as all the individual sources were well detected. We expect the amount of flux density missed by the small catalogue aperture to be negligible since the IRAC images do not show hints of resolved nebular emission at these wavelengths.

For each passband, the resulting flux densities from the stack analysis are plotted in the SEDs in Fig. 8 as blue circles. The top figure visualises the sum of all 18 candidate and confirmed LBVs in the LMC, while the bottom shows the same without RMC 143. In the top figure the pink symbols represent lower limits. The $70 \mu \mathrm{m}$ limit is given by the measurement in the stacked image without RMC 143, where the central source is better detected without the contamination by the 30 Dor region (as is evident from the comparison of the $70 \mu \mathrm{m}$ error bars in the top and bottom panels). The $250 \mu \mathrm{m}$ limit is due to a source detected at the central position of a stack image built from the only four well detected sources in this filter (namely RMC 127, LHA 120-S 119, LHA 120-S 61, and RMC 71). In the lower plot, the $160 \mu \mathrm{m}$ lower limit is set based on the detection in the stack image obtained from fewer 13 sources (IDs: $2,5,6-8,10,12-18$ ) that are less affected by crowding.

The total SED from about 1 to $250 \mu \mathrm{m}$ resembles that of LBV stars with dense ionised stellar winds, surrounded by extended dusty nebulae (Class 1a). We initially fit the 2MASS $J$, $H, K_{\mathrm{s}}$ and IRAC $3.6,4.5$ and $5.8 \mu \mathrm{m}$ data with a power law representing the sum of stellar winds and photospheres (powerlaw free-free emission from ionised stellar winds, Panagia \& Felli 1975 , and $F_{V} \propto \lambda^{-2}$ Rayleigh-Jeans photosphere emission), and then we added the resulting best fit and uncertainties to the grey-body function built to fit the WISE W3, MIPS24, MIPS70, PACS100, and PACS160 measurements. In the figure, the bestfit total model is given by a black continuous line. The powerlaw fit of the wind component is the blue dashed line. The grey shaded region represents all the grey-body components that fit the data producing a $\chi^{2}$ which is twice the minimum value of the best fit. Because of the large uncertainty of the $70 \mu \mathrm{m}$ produced by the crowded region around RMC 143, we set as lower limit the photometric measurement at $70 \mu \mathrm{m}$ without RMC 143 to constrain the range of possible models. We also introduce another constraint at longer wavelengths by using the lower limit 
Stack of 18 LBVs in the LMC
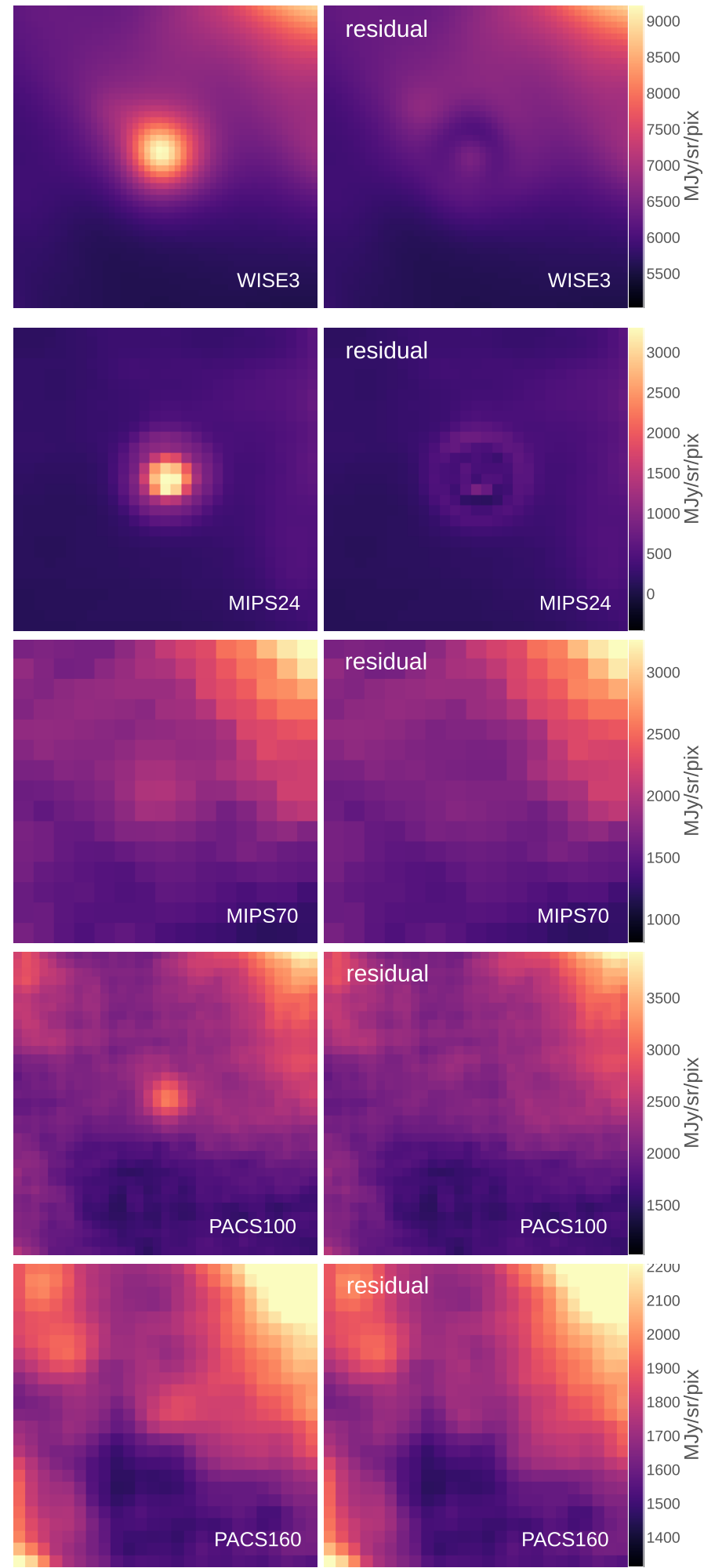
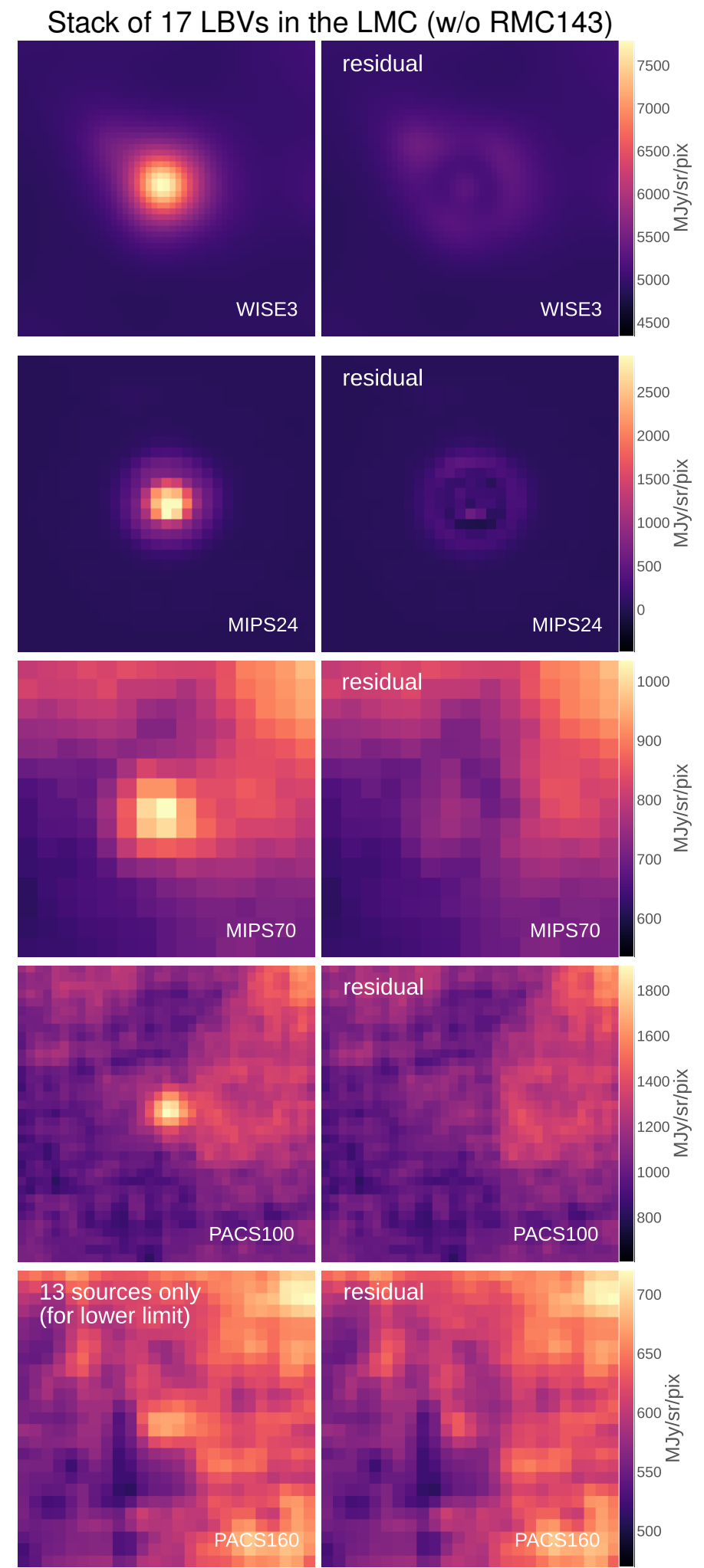

Fig. 7: Left column: stacked images of LMC LBVs (SED Classes 1 and 2 in Table 11) and residuals after Gaussian fitting. Right column: same as before but without RMC143. The field of view corresponds approximately to $70^{\prime \prime} \times 70^{\prime \prime}$. The stack image at $160 \mu \mathrm{m}$ on the bottom right was derived with a smaller number of sources (IDs: 2, 5, 6, 7, 8, 10,12,13,14,15,16,17, 18) and used as a lower limit.

at $250 \mu \mathrm{m}$. The resulting dust temperature, mass, and parameter $\beta$ are listed in the figures. The dust mass of the aggregate source is computed by assuming the flux density extracted from the best fit at $850 \mu \mathrm{m}$. The adopted absorption coefficient $\kappa_{850 \mu \mathrm{m}}$ is $1.7 \mathrm{~cm}^{2} \mathrm{~g}^{-1}$ (Sodroski et al. 1987). 

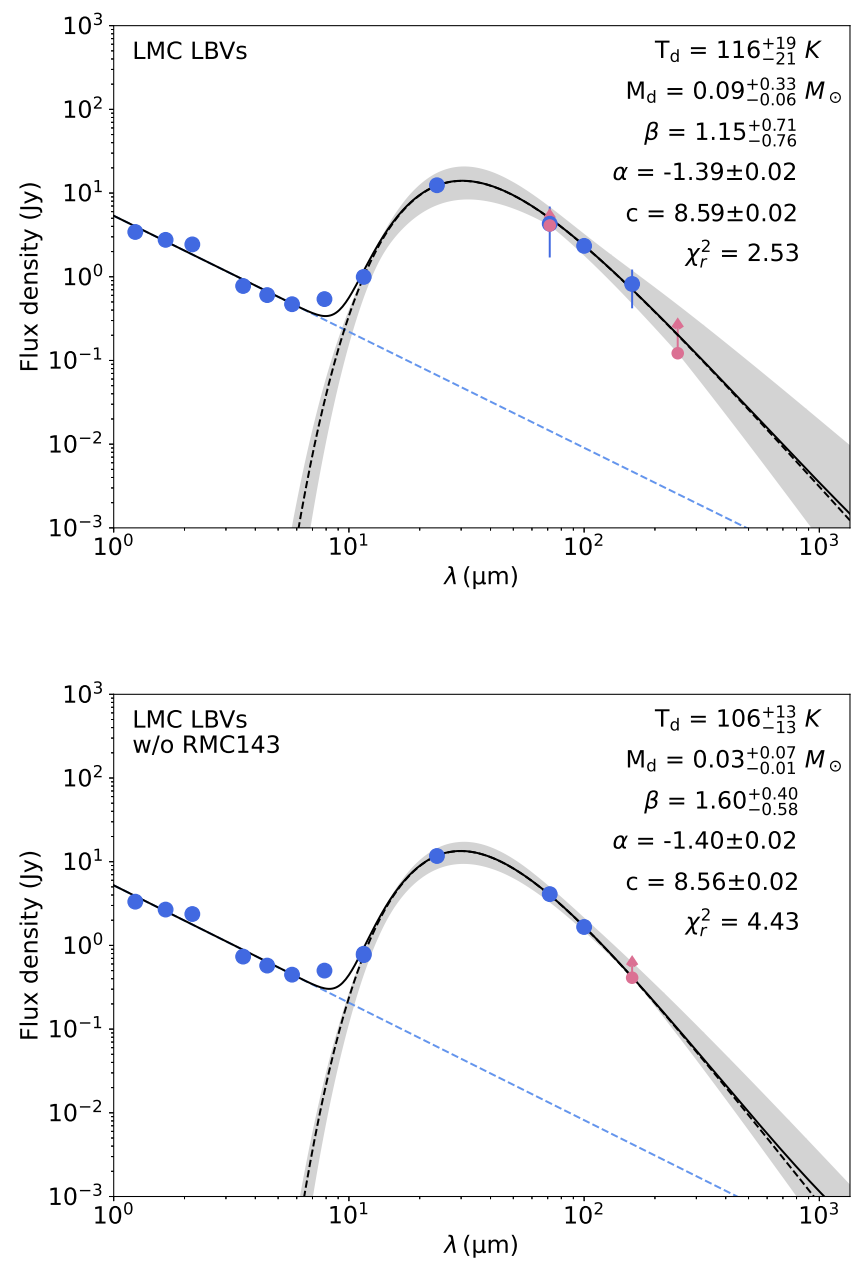

Fig. 8: Flux density distribution of stacks of the LBVs in the LMC (SED Classes 1 and 2 in Table 1), with (top) and without (bottom) RMC 143. 2MASS and IRAC values are the sum of the individual source fluxes, while WISE-W3, MIPS and PACS values are from photometry performed on the stacked images (Fig. 7). Parameters from the fitting procedure are shown ( $\alpha$ and $c$ are the spectral index and offset of the best-fit power-law describing the near-infrared data; $T_{\mathrm{d}}, M_{\mathrm{d}}$ and $\beta$ are the dust temperature, mass and opacity power law index of the best-fit greybody describing the thermal emission from dust). The lower limits (red points and arrow symbols) for MIPS70 and SPIRE250 in the upper plot and PACS160 in the lower plot are from stacks of subsets of sources (see text for the subsets in each case), as there is not a reliable detection in the stacked images of the whole sample.

\subsection{Stack analysis of other evolved stars in the LMC}

The same stacking analysis was performed for the sample of RSGs and AGBs in the catalogue of Riebel et al. (2012), which contains 33501 sources (6709 carbon-rich AGB, 19566 oxygenrich AGB, 5874 RSG and 1352 extreme "x-AGB" stars). Previously Dharmawardena (2019) stacked the PACS100 images of the sources in Riebel et al., split up by mass-loss rate bin and chemical class, and found some detections from carbon-rich and oxygen-rich AGB stars with the highest mass-loss rates. The wavelengths covered here are those of the four IRAC filters at 3.6, 4.5, 5.8 and $8 \mu \mathrm{m}$, the two MIPS filters at 24 and $70 \mu \mathrm{m}$, and the PACS filters at 100 and $160 \mu \mathrm{m}$. For completeness, we

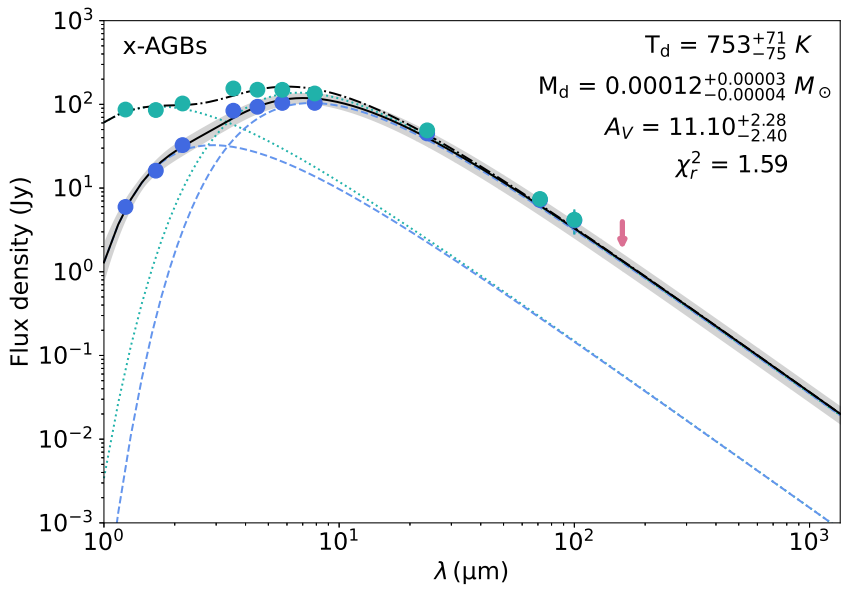

Fig. 9: Flux density distribution of the 1342 extreme $x$-AGBs in the LMC from the catalogue by Riebel et al. (2012), constructed from the Gaussian fitting of the central detection in the stacked images (at wavelengths shorter than $\sim 10 \mu \mathrm{m}$ the value is just the sum of the catalogue measurements). Blue points: observed phtometry; green points: de-reddened photometry; red arrow: $3 \sigma$ PACS160 upper limit.

also considered the SPIRE image at $250 \mu \mathrm{m}$, although with low expectation of obtaining a detection because of source confusion at those wavelengths. We cross-matched the list of LBVs and the catalogues of Riebel et al. and found two common sources (RMC 66 and RMC 84), which we removed from their sample (they were catalogued as RSGs). Various attempts were made to obtain a detection at wavelengths longer than $24 \mu \mathrm{m}$. Initially, the images of individual categories of stars were stacked separately, with and without applying a confusion threshold on the basis of the standard deviation evaluated from all the pixels in each field (measuring about $50 \times 50$ pixels). Finally, all the 33501 sources were summed together. In the $70 \mu \mathrm{m}$ and the stack images at longer wavelength, we did not obtain any statistically meaningful detection for the RSGs, the C- and O-rich AGBs, or the whole sample.

In the case of the "extreme" AGBs, which are considered the main dust producers among all AGBs and RSGs in the MCs (Boyer et al. 2012; Riebel et al. 2012), we evaluated possible detections at $24,100,160,250,350$ and $500 \mu \mathrm{m}$. We noticed that at far-infrared wavelengths the stacked images are dominated by confusion when the following fields are included: J053929.34694614.8, J053911.41-690824.7, J054515.83-694648.1, J051913.89-693818.3, J045506.54-691708.6. We also found that five other sources have been identified as YSOs or other types of stars (J053840.77-690603.3, J053856.58-690417.3, J053839.68-690537.9, J045400.16-691155.4, J053238.60682522.1, Evans et al. 2011; Walborn et al. 2013; van Gelder et al. 2020; Oliveira et al. 2019). We thus excluded these ten sources from further analysis. The final sample of extreme AGB stars from Riebel et al. (2012) that we stacked comprises 1342 objects. We obtained significant detections up to $100 \mu \mathrm{m}$, and a marginal detection at $160 \mu \mathrm{m}$, as shown in Fig. 10 . To produce the composite continuum spectrum in the figure, no weighting based on the stellar luminosity or normalisation was applied. The spectrum simply comprises the aggregated flux densities measured from the stacked images at each wavelength. 


\section{Stack of 1342 extreme AGBs in the LMC}
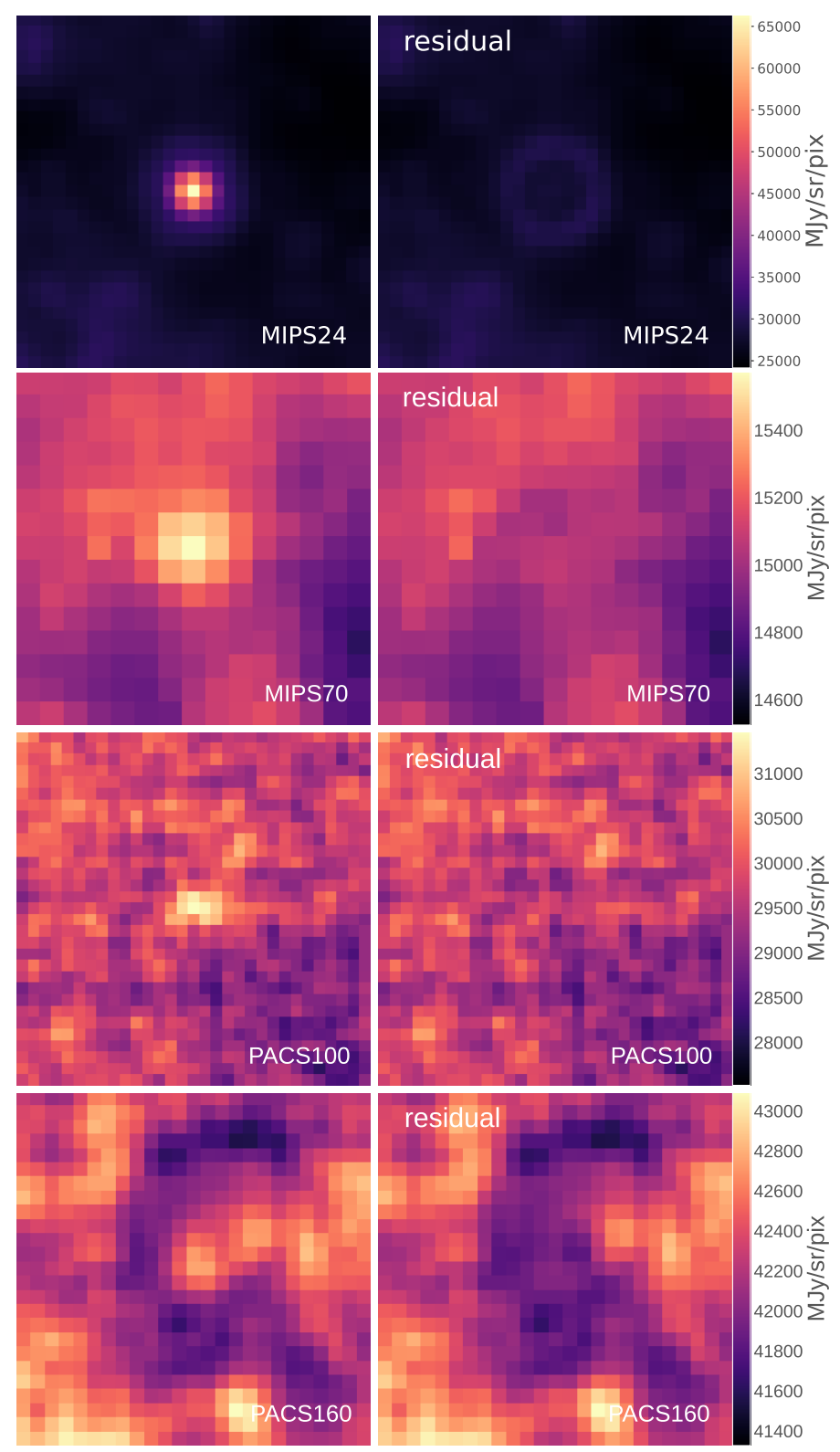

Fig. 10: Stacked images of the extreme-AGBs from Riebel et al. (2012) and residuals after Gaussian source extraction. The field of view corresponds approximately to $70^{\prime \prime} \times 70^{\prime \prime}$. Ten out of 1352 sources were excluded (see text), because the emission in their field is dominated by bright large-scale structures or because they are another type of stellar objects (e.g. YSOs).

We extracted the photometry as described in the preceding subsection. At $24 \mu \mathrm{m}$ we compared our photometry extracted from the stack image with the stacked photometry (obtained by summing the photometry in Riebel et al.2012) and found a consistent value. We fitted the data with two components: a black body of fixed temperature $(3000 \mathrm{~K})$ describing the stellar photosphere, and a grey body with dust spectral index $\beta=0$. Because the near-infrared data of AGBs are extremely reddened by the optically thick dust, we also fitted the extinction by using the extinction curve of Fitzpatrick (1999), with selective parameter $R_{V}=3.1$. Fig. 9 shows the photometry and resulting fit with and without extinction correction. For comparison, the integrated flux density at $100 \mu \mathrm{m}$ from $18 \mathrm{LBVs}$ is of the same order

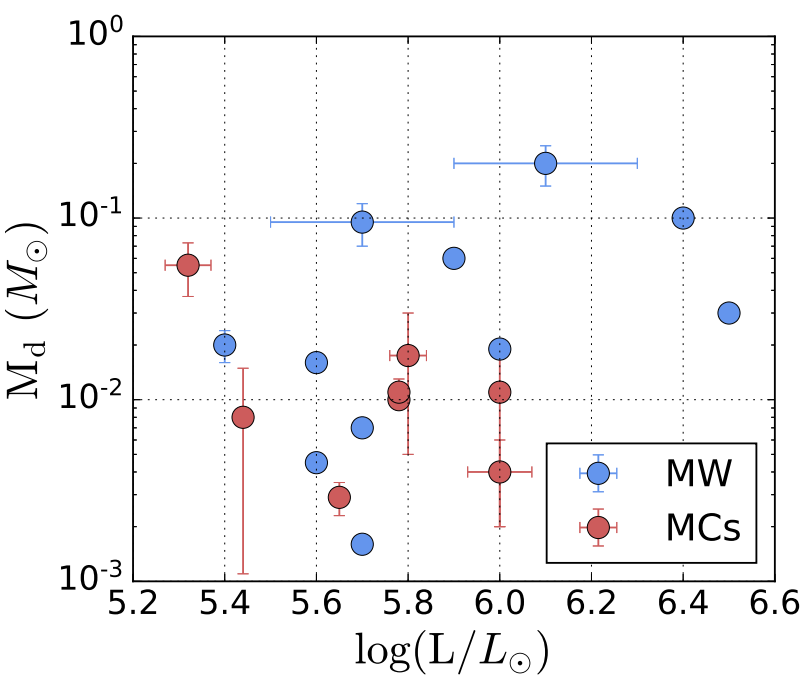

Fig. 11: Estimated dust masses for individual stars as a function of the stellar bolometric luminosity. Red circles: LMC LBVs (this work, see Table 6). Blue circles: Galactic LBVs, specifically AG Car (Vamvatira-Nakou et al. 2015); G24.73+0.69, G25.5+0.2, HD 168625, Hen 3-519, AFGL 2298 (Clark et al. 2003 , and references therein); G26.47+0.02 (Umana et al. 2012); G79.49+0.26 (Agliozzo et al. 2014); HR Car (Machado et al. 2002, Buemi et al. 2017); Pistol star (Lau et al. 2014); Wra 751 (Vamvatira-Nakou et al.2013). Error bars are shown when available from the literature.

of magnitude of that from 1342 extreme-AGB stars (see Table 7). The dust mass derived from this fitting assumes that the dust is optically thin (at the wavelength at which $\kappa$ is defined), and thus must be considered a lower limit.

\section{Discussion}

\subsection{LBV dust masses versus stellar luminosity and nebular size}

The uncertainty on the distance is only $\sim 2 \%$ for the Magellanic sources (see \$5.1), and thus their stellar luminosity is well constrained ( $\sim 4 \%$ uncertainty due to the distance). This is a great opportunity to investigate any possible dependence of the dust mass on the stellar parameters and on the nebular size. Fig. 11 shows the dust masses estimated in Sect.5.1 for 7 Class $1 \mathrm{LBVs}$ in the LMC and RMC 40 in the SMC versus the stellar bolometric luminosity as in the literature (Table A.1). In the same plot, dust masses for some well-known nebulae around candidate and confirmed LBVs in the Milky Way are displayed. Dust production in these stars does not seem correlated to the stellar properties.

For five objects the nebula size is known in the literature and one has been determined in this work. Sizes span from 0.3 to 2.2 pc (Table A.1). We do not observe a systematic trend in the dust mass versus the nebular size.

\subsection{Total dust mass in the LMC}

Since the ultimate goal of this work is to compare the LBV dust masses with the entire LMC dust yield we also fit the stack data with the simple grey model that Gordon et al. (2014) used to derive the global dust properties of the ISM in the LMC and SMC. In particular, we adopt their absorption coefficient 
Table 8: Summary of estimated LBV dust masses in the LMC.

\begin{tabular}{lcc}
\hline \hline Source & \multicolumn{2}{c}{ Grey-body fitting } \\
& $\left(\kappa_{\lambda}\right.$ as SD87) & $\left(\kappa_{\lambda}\right.$ as GD14 $)$ \\
\hline Stack 18 & $9_{-6}^{+33} \times 10^{-2} M_{\odot}$ & $11_{-3}^{+6} \times 10^{-2} M_{\odot}$ \\
Mean per source & $0.5_{-0.3}^{+1.8} \times 10^{-2} M_{\odot}$ & $0.6_{-0.2}^{+0.3} \times 10^{-2} M_{\odot}$ \\
\hline
\end{tabular}

Notes. Results are given for two different opacity values, as in Sodroski et al. 1987 (SD87) and Gordon et al. 2014 (GD14).

$\kappa_{\lambda}$ (calibrated with ultraviolet and optical gas-phase absorption measurements in the diffuse ISM of the Galaxy, which provide the expected amount of dust and are independent from the farinfrared and submillimetre dust emission). At $160 \mu \mathrm{m}$, Gordon et al. derived $\kappa_{160}=9.6 \pm 0.4 \pm 2.5$ where the first error is statistical and the second is the systematic uncertainty due to the assumption that the dust in the MCs is like that in the Galaxy. With this $\kappa_{160}$, our stack PACS160 flux and our stack SED fit temperature (top panel in Fig. 8), we obtain for the $18 \mathrm{LMC}$ (Class 1 and 2) LBVs $M_{\text {dust }}=0.11_{-0.03}^{+0.06} M_{\odot}$, and an average of $\left\langle M_{\text {dust }}\right\rangle=0.006_{-0.002}^{+0.003} M_{\odot}$ per source. The uncertainty of about $25 \%$ in $\kappa_{\lambda}$ due to the systematic error is not taken into account.

Table 8 summarises the dust masses derived from grey body fitting of the stack data. We notice that the integrated dust mass from 18 sources is of the same order of magnitude of the dust mass estimated in the Homunculus nebula of $\eta$ Carinae (Morris et al. 2017). This difference may be the result of different metallicities. It may also suggest that several stars have still not experienced the required mass loss to form dust (giant eruptions) or that they do not have colliding winds within a close binary. It should be noted that only a handful of sources contribute significantly to the stacked dust mass (compare Tables 6 and 8). Additionally, the dust masses estimated here could be a lower limit, as cooler dust may have escaped the observations. Sensitive sub-millimetre observations with ALMA of all Class 1 and 2 objects would be an asset for further studies.

For the LMC, Gordon et al. (2014) derived an integrated dust mass of $(7.3 \pm 1.7) \times 10^{5} M_{\odot}$ (averaging the results from three different models, or $(8.1 \pm 2.8) \times 10^{5} M_{\odot}$ with the simple grey body model only). A more recent analysis based on the same dataset reports estimates about a factor of two lower (Chastenet et al. 2017). In the classical scenario, the LBV phase is estimated to last around $10^{4}-10^{5} \mathrm{yr}$, which is a small fraction compared to the age of the LMC (about 15 Gyr, Harris \& Zaritsky 2009). To evaluate the potential contribution of LBVs to the ISM dust yield, we must account for the entire population of LBVs over the lifetime of the LMC.

This is not an easy task, because stellar evolution models are not able to predict how many BSGs become LBVs (or manifest the LBV phenomenon). Ideally, we would like to compare the ratio of LBVs and BSGs of similar luminosity, but finding a list near to completeness is challenging.

Urbaneja et al. (2017) analysed 90 LMC BSGs to study the gravity-luminosity relationship, but this number is probably a lower limit. In fact, in Bonanos et al. (2009) we count 133 stars earlier than B4 and of luminosity classes Ia and Iab in the LMC, excluding four objects that overlap with the LBV sample. However, Bonanos et al. (2009) could not claim completeness, as they targeted O, B supergiant stars with both accurate coordinates and spectral classification. We queried in Simbad for stars with spectral types earlier than B4 and of luminosity class I, within $2.5 \mathrm{deg}$ from the LMC centre, and we find around 230 BSGs (several of these objects appear in Massey 2002). Massey (2010) estimated $\sim 6100$ unevolved massive stars with $M>20 M_{\odot}$, and $\sim 700$ with $M>50 M_{\odot}$.

\subsubsection{Simple case: Constant number of LBVs over time}

The simplest estimate we can make of the total dust mass produced by LBVs in the LMC over all time is by assuming that the number of LBVs has on average been the same as today. This simple assumption avoids having to consider the OB to LBV rate, but it does depend directly on the assumed LBV phase duration. For the range of accepted LBV phase durations of $10^{4}-10^{5} \mathrm{yr}$, the LMC age of $15 \mathrm{Gyr}$, and the mass produced by the current LBV sample of $0.11_{-0.03}^{+0.06} M_{\odot}$, the range of total dust mass is from $1.2 \times 10^{4} M_{\odot}$ to $1.7 \times 10^{5} M_{\odot}$, with a nominal value of $6.6_{-5.4}^{+10.4} \times 10^{4} M_{\odot}$ for an assumed LBV phase duration of $25 \mathrm{kyr}$.

\subsubsection{Accounting for star formation history and initial mass function}

We assume that the LBV population closely followed the fluctuations in the star formation history (SFH) of the LMC. The latter was computed by Harris \& Zaritsky (2009), who derived star formation rates (SFR) in about $1400 \mathrm{LMC}$ sub-regions in time bins of $\Delta \log (t)=0.2$. We integrate the SFR over these sub-regions, in order to obtain the total SFH for the LMC as in Fig. 11 of Harris \& Zaritsky (2009). For the Initial Mass Function (IMF) we adopt the power law slope derived by Schneider et al. (2018), who found an excess of massive stars by $30 \%$ in the 30 Dor star forming region. We also use the Salpeter IMF as a possible lower limit in the LMC. Finally, we derive the total dust yield by LBVs as,

$$
\frac{\left(\sum_{i} \operatorname{SFR}\left(t_{i}\right) \Delta t_{i}\right) \times f_{\mathrm{LBV}, \mathrm{MS}} \times \int_{25 M_{\odot}}^{100 M_{\odot}} \xi(m) d m}{\int_{0.1 M_{\odot}}^{100 M_{\odot}} m \xi(m) d m} \times\left\langle M_{\mathrm{dust}}\right\rangle,
$$

where $\xi(m)=\xi_{0} \times m^{\gamma}$ is the $\operatorname{IMF} \operatorname{SFR}\left(t_{i}\right)$ is the SFR in the time bin $i,\left\langle M_{\text {dust }}\right\rangle$ is the mean dust mass produced per LBV, and $f_{\mathrm{LBV}, \mathrm{MS}}$ is the fraction of stars in mass range $25-100 M_{\odot}$ experiencing the LBV phenomenon. The IMF is normalised to the total mass of stars with masses between 0.1 and $100 M_{\odot}$. Assuming the slope $\gamma=-1.9$ from Schneider et al. (2018) (Case 1) and $\gamma=-2.35$ from Salpeter (Case 2), we find the following ranges of dust mass: $17.1_{-3.7}^{+14.0} \times 10^{4} \times f_{\mathrm{LBV}, \mathrm{MS}} M_{\odot}$ (Case 1) and $4.4_{-1.0}^{+3.6} \times 10^{4} \times f_{\mathrm{LBV}, \mathrm{MS}} M_{\odot}$ (Case 2$)$. The error bars include the uncertainty on the dust mass and on the SFR. A major uncertainty in this relation lies in the fraction of massive mainsequence stars that will become LBV stars $\left(f_{\mathrm{LBV}, \mathrm{MS}}\right)$. Obviously, the number cannot exceed unity. Because the conditions leading to the LBV phenomenon are unknown, it is unconstrained by evolutionary models.

The observed fraction with respect to BSGs, $f_{\mathrm{LBV}, \mathrm{obs}}=$ $n_{\mathrm{LBV}, \mathrm{obs}} / n_{\mathrm{BSG}, \mathrm{obs}}$, needs to be corrected for incompleteness and, more importantly, for the ratio of the duration of the BSG and LBV phases, $t_{\mathrm{BSG}} / t_{\mathrm{LBV}}$. Evolutionary models suggest $t_{\mathrm{BSG}} \leq$ $10^{6} \mathrm{yr}$ for stars more massive than $25 M_{\odot}$ in the He-burning phase, and $t_{\mathrm{MS}} \leq 10^{7} \mathrm{yr}$ for stars in the H-burning phase (Maeder \& Meynet 1989). While the number of stars in the LMC currently exhibiting the LBV phenomenon should be reasonably 
correct $\left(n_{L B V, o b s}\right)$, it should be subject to fluctuations, and some current LBVs might be inactive. The number of BSGs in the LMC that may become LBVs ( $n_{\mathrm{BSG}, \mathrm{obs}} \sim 230$, see above) is also uncertain. The number of MS O,B stars (6700) may be more reliable (Massey 2002, 2010). From the currently available information, it seems plausible that $f_{\mathrm{LBV}, \mathrm{MS}}$ ranges between about $30 \% 5$ and $100 \% 6$

We repeat a similar calculation for the AGB/RSG stars. We use the average dust mass per star determined from the stack of all extreme-AGBs $\left(9_{-3.0}^{+2.2} \times 10^{-8} M_{\odot}\right)$ and we integrate over stellar masses between 1 and $25 M_{\odot}$ to also include RSGs. We find a total dust mass of $136_{-35}^{+56} M_{\odot}$ (Case 1) or $144_{-37}^{+59} M_{\odot}$ (Case 2), which are much lower values than LBVs, but maybe consistent with the long dust injection timescales of AGBs $\left(\sim 2 \times 10^{9} \mathrm{yr}^{-1}\right.$ Micelotta et al. 2018). Moreover, the dust lifetime against processing and destruction by supernova shocks in the ISM is shorter, around $2 \times 10^{7} \mathrm{yr}$ (Temim et al. 2015). The inefficiency of existing AGB stars to produce the dust mass observed in the ISM was already pointed out by Matsuura et al. (2009) for the LMC and by Boyer et al. (2012) and Matsuura et al. (2013) for the SMC. Dust injection timescales from LBVs (and subsequent $\mathrm{SNe})$ are much shorter $(\leq 10-100 \mathrm{Myr})$. In their study of the total dust input from stars in the SMC Boyer et al. (2012) estimated a dust production rate of $\sim 10^{-6} M_{\odot} \mathrm{yr}^{-1}$ from LBVs, matching the input from all cool stars, but concluded that the grains would not survive the subsequent supernova explosion.

\subsection{Potential dust survival from SN explosions}

Now we evaluate how likely this LBV dust is to survive the SN forward shock (blast wave) which will follow the LBV or WR evolutionary stage. The case of ISM grains processing by the forward shock was analysed by Barlow (1978), Nozawa et al. (2006), and Bocchio et al. (2014). The processes of interest are generally sputtering and shattering. There are a few arguments to mention in favour of dust survival in the SN blast wave in LBVNe. 1) LBV eruptions are a source of large grains $(>0.1 \mu \mathrm{m})$ due to the favourable conditions for particle growth (Kochanek 2011b). The relatively low $\beta$ values found in this work are also consistent with large grains. However, they could also be low due to a temperature gradient over the nebula. 2) The material in LBVNe seems often distributed in a clumpy configuration, spread at large distances from the star. 3) Such nebulae can be asymmetric (e.g. RMC 143, LHA 120-S 119), subtending solid angles of less than $4 \pi$ so that some of the SN energy will leak out without interacting with the nebula.

Such properties reduce the importance of the destructive action by the shock, as for example evidenced in studies of the newly formed dust in SN ejecta impacted by the reverse shock. In particular, survival rates vary between 40 and $98 \%$ (Nath et al.2008, Silvia et al.|2010, 2012, Biscaro \& Cherchneff 2016, Gall \& Hjorth 2018) for dust reached by the reverse shock, and around $85 \%$ for large grains in the ISM (e.g. Nozawa et al. 2006). More results are summarised in Table 2 of Micelotta et al. (2018). Some studies also show that shocks can induce

\footnotetext{
5 Comparing the LBVs with the MS OBs, taking $10^{7} \mathrm{yr}$ as the MS lifetime and an upper limit of $10^{5} \mathrm{yr}$ for the LBV phase duration gives, $f_{\mathrm{LBV}}=(18 \mathrm{LBVs} / 6700 \mathrm{MSOBs}) \times\left(10^{7} \mathrm{yr} / 10^{5} \mathrm{yr}\right)=27 \%$.

${ }^{6}$ Comparing the LBVs with the BSGs, taking $10^{6} \mathrm{yr}$ as the BSG phase duration and $10^{5} \mathrm{yr}$ for the LBV phase duration gives,

$(18 \mathrm{LBVs} / 230 \mathrm{BSGs}) \times\left(10^{6} \mathrm{yr} / 10^{5} \mathrm{yr}\right)=78 \%$.

As the LBV phase may be an order of magnitude shorter than this, it suggests that the number of 230 BSGs may be incomplete.
}

dust (re)formation and growth (e.g. Kochanek 2011a; Matsuura et al. 2019). Recent 3-D hydrodynamical simulations report that the wind-driven bubbles created during the entire life of a massive star create the conditions for the dust to largely survive (Martínez-González et al. 2019), or that under favourable ambient conditions implantation and trapping of ions in dust grains can counteract grain destruction by sputtering (Kirchschlager et al. 2020). Dust destruction is particularly negligible if the preexisting shell is massive (Martínez-González et al.2019), which is the case of LBV nebulae.

From the observational point of view, a great example is SN1987A, the progenitor of which may have been an LBV (as speculated by Smith 2007). Along with the discovery of dust formation in the $\mathrm{SN}$ ejecta a few years after the explosion (Danziger et al. 1989), followed up by far-infrared and sub-millimetre observations about three decades later (which revealed large amounts $\sim 0.5 M_{\odot}$ of cold dust, Matsuura et al. 2011, Indebetouw et al. 2014; Wesson et al. 2015), another dust component distributed in an equatorial ring lost by the progenitor 20 - 40 kyr ago was found (Arnett et al. 1989; McCray|1993. Bouchet et al. 2006). The fastest part of the forward shock has passed the ring (Fransson et al. 2015), offering unique insights into the resulting dust destructive processing. The silicate grains in the ring are collisionally heated by the SN blast wave (Bouchet et al. 2006). Arendt et al. (2016) proposed that some dust grains were destroyed by sputtering, supporting the theory (Dwek et al. 2008. 2010). Subsequently, Matsuura et al. (2019) reported a $30-70 \mu \mathrm{m}$ excess at day $\sim 10000$ on top of the earlier modelled hot and warm component (in the ring) and cold component (in the SN ejecta), and a dust mass about a factor of 10 larger than previous estimates at day $\sim 8000$. They identified two possible mechanisms behind this infrared excess: dust re-formation (dust grains formed from the gas phase) and dust growth (survived grains accreting atoms from the gas phase). Dust re-formation was also suggested in the cool dense shell created between the forward and reverse shocks as the SN ejecta plows into the existing circumstellar material. Several studies of type IIn $\mathrm{SNe}$ (which, in some cases, may have an LBV as progenitor) are a good example (e.g. Smith et al. 2008b; Fox et al. 2009; Gall et al. 2014, Andrews et al. 2016, Chugai 2018). Grain growth may also be a viable mechanism as explained by Kirchschlager et al. (2020).

\section{Summary and conclusions}

We have analysed the infrared images of Magellanic LBVs available in the archives, adding for a few sources new photometry that either was missing or not accurate because of extended objects or crowded fields. We have revised the Magellanic LBV sample on the basis of a new infrared SED-based classification scheme and literature information. The sources can be divided in four classes. All strongly active LBVs show either a cool dust excess peaking in the mid-infrared (Class 1a and 1b) or an excess of near-infrared emission due to free-free emission from the ionised stellar wind (Class 2), or both (Class 1a). These features are also often present in several candidate LBVs, which appear in the literature as ex-/dormant LBVs. Four sources can be removed from our initial sample, as we find that in past studies these stars did not satisfy the LBV requirements and, in addition, they do not present any of the features mentioned above (Class 4). Six other sources are known as sgB[e] (Class 3): their flux density distribution does not resemble those of confirmed LBVs, consistent with a different mechanism of dust formation, 
as suggested in the literature. For this reason we excluded them from further analysis.

We have employed a simple grey-body fitting method to model the infrared SED of individual sources. In the LMC, which has the most numerous list of LBVs, large amounts of dust are observed $\left(\sim 10^{-3}-10^{-2} M_{\odot}\right)$, similar to Galactic LBVNe. We stacked the infrared images of the LMC LBVs and extracted the photometry of the resulting source, detected up to $160-250 \mu \mathrm{m}$. The integrated SED from the stacks resembles that of LBVs with a strong ionised stellar wind and an extended dusty nebula. The SED can be fitted with only two components: a power-law describing the free-free spectrum of ionised stellar winds and the stellar photosphere, and a single-component grey-body for the dust. For the grey-body we adopted two different values of the $\kappa$ parameter, including the value determined by Gordon et al. (2014) to fit the integrated ISM SED of the LMC. A significant contribution to the stack SED comes from a few sources, the most important one is RMC143. This was already identified as a massive nebula (Agliozzo et al. 2019). We obtain an integrated present dust mass of $0.11_{-0.03}^{+0.06} M_{\odot}$. We have repeated a similar analysis on the sample of AGBs and RSGs by Riebel et al. (2012). We obtain a detection in the stacked images only when considering the "extreme"-AGBs. We find that the integrated $160 \mu \mathrm{m}$ emission of 1342 extreme-AGBs is of the same order of magnitude as that of 18 LBVs. The integrated dust mass from these sources is $1.2_{-0.4}^{+0.3} \times 10^{-4} M_{\odot}$. We do not find any correlation between the dust masses and the stellar luminosities. This could be due to the fact that such stars have different evolutionary histories or that the dust production mechanism does not depend on the initial mass of the star. Most likely we are also unable to detect the lowest-mass nebulae. To estimate the total dust mass produced by LBVs in the LMC during its full lifetime, we consider two cases: constant number of LBVs across time and a case accounting for IMF and SFH. The uncertainty on the duration of LBV phase in the first case, or on the population of LBVs in the second case, add a significant uncertainty in the total mass produced by LBVs.

The range of total dust masses produced by LBVs over the lifetime of the LMC spans between $10^{4}$ and $10^{5} M_{\odot}$, suggesting that LBVs are potentially significant dust factories in the LMC. The LBV dust masses per source of $10^{-3}-10^{-2} M_{\odot}$ are at least one order of magnitude smaller than the amount of dust $\left(0.1-1 M_{\odot}\right)$ postulated in SN ejecta by Todini \& Ferrara (2001) and Nozawa et al. (2003). However, those works adopted ideal assumptions for the seed material condensing into dust grains. Mid-infrared observations of CC-SNe suggest smaller dust masses $\left(10^{-4}-10^{-2} M_{\odot}\right.$, Kotak et al. 2009, Fox et al. 2011, although estimates from single epoch observations of the rapidly evolving SN ejecta may not be representative of the total dust produced or mid-infrared observations may have not captured the emission from colder dust). On the other hand ALMA submillimetre observations revealed larger amounts of dust in both SN1987A $\left(0.2 M_{\odot}\right.$, Indebetouw et al. 2014) and LBV RMC143 $\left(0.055 M_{\odot}\right.$, Agliozzo et al. 2019), however these works assume different opacities at $850 \mu \mathrm{m}$, making the comparison difficult. Still the LBV phenomenon has the potential to be the second most important source of dust, before low- and intermediatemass stars. This was also the conclusion by Boyer et al. (2012) in the SMC. It should be noted that if, on the one hand, the LBV dust mass has a large uncertainty after analysing a relatively large sample, in the case of CC-SNe the dust mass is also very uncertain and based on observations of only a handful of objects. Destruction of dust in LBVNe by a possible subsequent SN blast wave remains to be determined. A significant fraction of this dust mass may survive thanks to several properties of these nebulae, such as large nebular masses, big grains, clumpiness and asymmetry. Dust grain (re)formation or growth may also occur, like in the case of SN1987A (Matsuura et al.2019).

Acknowledgements. D.A. acknowledges funding through the European Union's Horizon 2020 and Innovation programme under the Marie Skłodowska-Curie grant agreement no. 793499 (DUSTDEVILS). F.K. is supported by the Ministry of Science and Technology (MoST) of Taiwan, under grant number MOST1072119-M-001-031-MY3, and also by Academia Sinica, in the form of Investigator Award AS-IA-106-MO3. GP is supported by ANID - Millennium Science Initiative - ICN12_009. This paper makes use of the following ALMA data: ADS/JAO.ALMA\#2017.A.00054.S and ADS/JAO.ALMA\#2018.A.00049.S. ALMA is a partnership of ESO (representing its member states), NSF (USA) and NINS (Japan), together with NRC (Canada) and NSC and ASIAA (Taiwan) and KASI (Republic of Korea), in cooperation with the Republic of Chile. The Joint ALMA Observatory is operated by ESO, AUI/NRAO and NAOJ. This paper also includes data collected: at the European Organisation for Astronomical Research in the Southern Hemisphere under ESO programmes 096.D-0047(A), 097.D0612(A,B), and 0100.D-0469(A,B). This work made use of PyAstronomy. This research has made use of the International Variable Star Index (VSX) database, operated at AAVSO, Cambridge, Massachusetts, USA, and the VizieR catalogue access tool, CDS, Strasbourg, France. The original description of the VizieR service was published in A\&AS 143, 23. This research has made use of the NASA/IPAC Infrared Science Archive, which is funded by the National Aeronautics and Space Administration and operated by the California Institute of Technology. This research made use of Photutils, an Astropy package for detection and photometry of astronomical sources (Bradley et al. 2021). The Cornell Atlas of Spitzer/IRS Sources (CASSIS) is a product of the Infrared Science Center at Cornell University, supported by NASA and JPL.

\section{References}

Agliozzo, C., Mehner, A., Phillips, N. M., et al. 2019, A\&A, 626, A126 Agliozzo, C., Nikutta, R., Pignata, G., et al. 2017a, MNRAS, 466, 213

Agliozzo, C., Noriega-Crespo, A., Umana, G., et al. 2014, MNRAS, 440, 1391 Agliozzo, C., Trigilio, C., Pignata, G., et al. 2017b, ApJ, 841, 130 Agliozzo, C., Umana, G., Trigilio, C., et al. 2012, MNRAS, 426, 181 Andrews, J. E., Krafton, K. M., Clayton, G. C., et al. 2016, MNRAS, 457, 3241 Ardila, D. R., Van Dyk, S. D., Makowiecki, W., et al. 2010, ApJS, 191, 301 Arendt, R. G., Dwek, E., Bouchet, P., et al. 2016, AJ, 151, 62

Aret, A., Kraus, M., Muratore, M. F., \& Borges Fernand es, M. 2012, MNRAS, 423, 284

Arneson, R. A., Shenoy, D., Smith, N., \& Gehrz, R. D. 2018, ApJ, 864, 31

Arnett, W. D., Bahcall, J. N., Kirshner, R. P., \& Woosley, S. E. 1989, ARA\&A, 27, 629

Barba, R. H., Morrell, N. I., Niemela, V. S., et al. 1996, in Revista Mexicana de Astronomia y Astrofisica Conference Series, Vol. 5, Revista Mexicana de Astronomia y Astrofisica Conference Series, ed. V. Niemela, N. Morrell, P. Pismis, \& S. Torres-Peimbert, 85-91

Barlow, M. J. 1978, MNRAS, 183, 367

Beichman, C. A., Neugebauer, G., Habing, H. J., Clegg, P. E., \& Chester, T. J. 1988, Infrared astronomical satellite (IRAS) catalogs and atlases. Volume 1: Explanatory supplement, Vol. 1 (The Joint IRAS Science Working Group)

Biscaro, C. \& Cherchneff, I. 2016, A\&A, 589, A132

Bocchio, M., Jones, A. P., \& Slavin, J. D. 2014, A\&A, 570, A32

Boffin, H. M. J., Rivinius, T., Mérand, A., et al. 2016, A\&A, 593, A90

Boian, I. \& Groh, J. H. 2018, A\&A, 617, A115

Bonanos, A. Z., Lennon, D. J., Köhlinger, F., et al. 2010, AJ, 140, 416

Bonanos, A. Z., Massa, D. L., Sewilo, M., et al. 2009, AJ, 138, 1003

Bouchet, P., Dwek, E., Danziger, J., et al. 2006, ApJ, 650, 212

Boyer, M. L., Srinivasan, S., Riebel, D., et al. 2012, ApJ, 748, 40

Buemi, C. S., Trigilio, C., Leto, P., et al. 2017, MNRAS, 465, 4147

Buemi, C. S., Umana, G., Trigilio, C., Leto, P., \& Hora, J. L. 2010, ApJ, 721, 1404

Campagnolo, J. C. N., Borges Fernandes, M., Drake, N. A., et al. 2018, A\&A, 613, A33

Castor, J. I., Abbott, D. C., \& Klein, R. I. 1975, ApJ, 195, 157

Chastenet, J., Bot, C., Gordon, K. D., et al. 2017, A\&A, 601, A55

Chugai, N. N. 2018, MNRAS, 481, 3643

Clark, J. S., Bartlett, E. S., Coe, M. J., et al. 2013, A\&A, 560, A10

Clark, J. S., Egan, M. P., Crowther, P. A., et al. 2003, A\&A, 412, 185

Cohen, M., Walker, R. G., Carter, B., et al. 1999, AJ, 117, 1864

Conti, P. S. 1984, in Observational Tests of the Stellar Evolution Theory, ed.

A. Maeder \& A. Renzini, Vol. 105, 233

Crawford, T. M., Chown, R., Holder, G. P., et al. 2016, ApJS, 227, 23 
Crowther, P. A. 2007, ARA\&A, 45, 177

Crowther, P. A. \& Smith, L. J. 1997, A\&A, 320, 500

Cutri, R. M., Skrutskie, M. F., van Dyk, S., et al. 2003, VizieR Online Data Catalog, 2246

Cutri, R. M., Skrutskie, M. F., van Dyk, S., et al. 2012, VizieR Online Data Catalog, 2281

Cutri, R. M., Wright, E. L., Conrow, T., et al. 2021, VizieR Online Data Catalog, II/328

Danforth, C. W. \& Chu, Y.-H. 2001, ApJ, 552, L155

Danziger, I. J., Gouiffes, C., Bouchet, P., \& Lucy, L. B. 1989, IAU Circ., 4746, 1

de Wit, W. J., Oudmaijer, R. D., \& Vink, J. S. 2014, Advances in Astronomy, 2014,270848

Dharmawardena, T. E. 2019, PhD thesis, National Central University, Taiwan

Dole, H., Lagache, G., Puget, J. L., et al. 2006, A\&A, 451, 417

Draine, B. T. 2009, in Astronomical Society of the Pacific Conference Series, Vol. 414, Cosmic Dust - Near and Far, ed. T. Henning, E. Grün, \& J. Steinacker, 453

Dwek, E., Arendt, R. G., Bouchet, P., et al. 2008, ApJ, 676, 1029

Dwek, E., Arendt, R. G., Bouchet, P., et al. 2010, ApJ, 722, 425

Egan, M. P., Clark, J. S., Mizuno, D. R., et al. 2002, ApJ, 572, 288

Egan, M. P., Price, S. D., Kraemer, K. E., et al. 2003, VizieR Online Data Cata$\log , \mathrm{V} / 114$

Elias-Rosa, N., Benetti, S., Cappellaro, E., et al. 2018, Monthly Notices of the Royal Astronomical Society, 475, 2614

Enard, D. 1991, Journal of Optics, 22, 33

Evans, C. J., Crowther, P. A., Fullerton, A. W., \& Hillier, D. J. 2004, ApJ, 610, 1021

Evans, C. J., Taylor, W. D., Hénault-Brunet, V., et al. 2011, A\&A, 530, A108

Fariña, C., Bosch, G. L., Morrell, N. I., Barbá, R. H., \& Walborn, N. R. 2009, AJ, 138,510

Feast, M. W., Thackeray, A. D., \& Wesselink, A. J. 1960, MNRAS, 121, 337

Fitzpatrick, E. L. 1991, PASP, 103, 1123

Fitzpatrick, E. L. 1999, PASP, 111, 63

Fox, O., Skrutskie, M. F., Chevalier, R. A., et al. 2009, ApJ, 691, 650

Fox, O. D., Chevalier, R. A., Skrutskie, M. F., et al. 2011, ApJ, 741, 7

Fransson, C., Larsson, J., Migotto, K., et al. 2015, ApJ, 806, L19

Gaia Collaboration. 2018, VizieR Online Data Catalog, I/345

Gal-Yam, A. \& Leonard, D. C. 2009, Nature, 458, 865

Gall, C. \& Hjorth, J. 2018, ApJ, 868, 62

Gall, C., Hjorth, J., Watson, D., et al. 2014, Nature, 511, 326

Gallagher, J. S. 1989, in Astrophysics and Space Science Library, Vol. 157, IAU Colloq. 113: Physics of Luminous Blue Variables, ed. K. Davidson, A. F. J. Moffat, \& H. J. G. L. M. Lamers, 185-192

Gordon, K. D., Roman-Duval, J., Bot, C., et al. 2014, ApJ, 797, 85

Graczyk, D., Pietrzyński, G., Thompson, I. B., et al. 2020, ApJ, 904, 13

Gräfener, G., Owocki, S. P., \& Vink, J. S. 2012, A\&A, 538, A40

Grassitelli, L., Langer, N., Mackey, J., et al. 2021, A\&A, 647, A99

Griffin, M. J., Abergel, A., Abreu, A., et al. 2010, A\&A, 518, L3

Groh, J. H. 2014, A\&A, 572, L11

Groh, J. H., Damineli, A., Hillier, D. J., et al. 2009, ApJ, 705, L25

Groh, J. H., Meynet, G., \& Ekström, S. 2013, A\&A, 550, L7

Guha Niyogi, S., Min, M., Meixner, M., et al. 2014, A\&A, 569, A80

Gvaramadze, V. V., Kniazev, A. Y., \& Fabrika, S. 2010, MNRAS, 405, 1047

Gvaramadze, V. V., Kniazev, A. Y., Maryeva, O. V., \& Berdnikov, L. N. 2018, MNRAS, 474, 1412

Hainich, R., Rühling, U., Todt, H., et al. 2014, A\&A, 565, A27

Harris, J. \& Zaritsky, D. 2009, AJ, 138, 1243

Houck, J. R., Roellig, T. L., van Cleve, J., et al. 2004, ApJS, 154, 18

Humphreys, R. M. \& Davidson, K. 1994, PASP, 106, 1025

Hutsemekers, D. 1997, in Astronomical Society of the Pacific Conference Series, Vol. 120, Luminous Blue Variables: Massive Stars in Transition, ed. A. Nota \& H. Lamers, 316

Indebetouw, R., Matsuura, M., Dwek, E., et al. 2014, ApJ, 782, L2

Ishihara, D., Onaka, T., Kataza, H., et al. 2010, A\&A, 514, A1

Jones, O. C., Meixner, M., Sargent, B. A., et al. 2015, ApJ, 811, 145

Jones, O. C., Woods, P. M., Kemper, F., et al. 2017, MNRAS, 470, 3250

Kastner, J. H., Buchanan, C., Sahai, R., Forrest, W. J., \& Sargent, B. A. 2010, AJ, 139, 1993

Kastner, J. H., Buchanan, C. L., Sargent, B., \& Forrest, W. J. 2006, ApJ, 638, L29

Kato, D., Ita, Y., Onaka, T., et al. 2012, AJ, 144, 179

Käufl, H. U., Kerber, F., Asmus, D., et al. 2015, The Messenger, 159, 15

Kemper, F., Woods, P. M., Antoniou, V., et al. 2010, PASP, 122, 683

Kerber, F., Käufl, H. U., Tristram, K., et al. 2016, in Society of Photo-Optical Instrumentation Engineers (SPIE) Conference Series, Vol. 9908, Ground-based and Airborne Instrumentation for Astronomy VI, 99080D

Kirchschlager, F., Barlow, M. J., \& Schmidt, F. D. 2020, ApJ, 893, 70

Kochanek, C. S. 2011a, ApJ, 741, 37

Kochanek, C. S. 2011b, ApJ, 743, 73
Koenigsberger, G., Auer, L. H., Georgiev, L., \& Guinan, E. 1998, ApJ, 496, 934 Koenigsberger, G., Morrell, N., Hillier, D. J., et al. 2014, AJ, 148, 62 Kotak, R., Meikle, W. P. S., Farrah, D., et al. 2009, ApJ, 704, 306

Kotak, R. \& Vink, J. S. 2006, A\&A, 460, L5

Kurczynski, P. \& Gawiser, E. 2010, AJ, 139, 1592

Lagage, P. O., Pel, J. W., Authier, M., et al. 2004, The Messenger, 117, 12

Laporte, N., Ellis, R. S., Boone, F., et al. 2017, ApJ, 837, L21

Lau, R. M., Eldridge, J. J., Hankins, M. J., et al. 2020, ApJ, 898, 74

Lau, R. M., Herter, T. L., Morris, M. R., \& Adams, J. D. 2014, ApJ, 785, 120

Lebouteiller, V., Barry, D. J., Goes, C., et al. 2015, ApJS, 218, 21

Lebouteiller, V., Barry, D. J., Spoon, H. W. W., et al. 2011, ApJS, 196, 8

Machado, M. A. D., de Araújo, F. X., Pereira, C. B., \& Fernandes, M. B. 2002, A\&A, 387, 151

Maeder, A. 1989, in Astrophysics and Space Science Library, Vol. 157, IAU Colloq. 113: Physics of Luminous Blue Variables, ed. K. Davidson, A. F. J. Moffat, \& H. J. G. L. M. Lamers, 15

Maeder, A. \& Meynet, G. 1989, A\&A, 210, 155

Margutti, R., Milisavljevic, D., Soderberg, A. M., et al. 2014, ApJ, 780, 21

Martínez-González, S., Wünsch, R., Silich, S., et al. 2019, arXiv e-prints, arXiv:1911.05079

Massey, P. 2002, ApJS, 141, 81

Massey, P. 2010, in Astronomical Society of the Pacific Conference Series, Vol. 425, Hot and Cool: Bridging Gaps in Massive Star Evolution, ed. C. Leitherer, P. D. Bennett, P. W. Morris, \& J. T. Van Loon, 3

Massey, P., McNeill, R. T., Olsen, K. A. G., et al. 2007, AJ, 134, 2474

Matsuura, M., Barlow, M. J., Zijlstra, A. A., et al. 2009, MNRAS, 396, 918

Matsuura, M., De Buizer, J. M., Arendt, R. G., et al. 2019, MNRAS, 482, 1715

Matsuura, M., Dwek, E., Barlow, M. J., et al. 2015, ApJ, 800, 50

Matsuura, M., Dwek, E., Meixner, M., et al. 2011, Science, 333, 1258

Matsuura, M., Woods, P. M., \& Owen, P. J. 2013, MNRAS, 429, 2527

Mauerhan, J. C., Smith, N., Filippenko, A. V., et al. 2013, Monthly Notices of the Royal Astronomical Society, 430, 1801

McCray, R. 1993, ARA\&A, 31, 175

McKinnon, R., Torrey, P., \& Vogelsberger, M. 2016, MNRAS, 457, 3775

Mehner, A., Baade, D., Groh, J. H., et al. 2017, A\&A, 608, A124

Mehner, A., Baade, D., Rivinius, T., et al. 2013, A\&A, 555, A116

Meixner, M., Gordon, K. D., Indebetouw, R., et al. 2006, AJ, 132, 2268

Meixner, M., Panuzzo, P., Roman-Duval, J., et al. 2013, AJ, 146, 62

Micelotta, E. R., Matsuura, M., \& Sarangi, A. 2018, Space Sci. Rev., 214, 53

Morris, P. W., Gull, T. R., Hillier, D. J., et al. 2017, ApJ, 842, 79

Muratorio, G. \& Friedjung, M. 1988, A\&A, 190, 103

Nath, B. B., Laskar, T., \& Shull, J. M. 2008, ApJ, 682, 1055

Neugent, K. F., Massey, P., Skiff, B., \& Meynet, G. 2012, ApJ, 749, 177

Nozawa, T., Kozasa, T., \& Habe, A. 2006, ApJ, 648, 435

Nozawa, T., Kozasa, T., Umeda, H., Maeda, K., \& Nomoto, K. 2003, ApJ, 598, 785

Ochsenbein, F., Bauer, P., \& Marcout, J. 2000, A\&AS, 143, 23

Oliveira, J. M., van Loon, J. T., Sewiło, M., et al. 2019, MNRAS, 490, 3909

Osmer, P. S. 1973, ApJ, 186, 459

Owocki, S. P., Townsend, R. H. D., \& Quataert, E. 2017, MNRAS, 472, 3749

Panagia, N. \& Felli, M. 1975, A\&A, 39, 1

Pasquali, A., Schmutz, W., Nota, A., \& Origlia, L. 1997, A\&A, 327, 265

Pastorello, A., Kochanek, C. S., Fraser, M., et al. 2017, Monthly Notices of the Royal Astronomical Society, 474, 197

Pietrzyński, G., Graczyk, D., Gieren, W., et al. 2013, Nature, 495, 76

Poglitsch, A., Waelkens, C., Geis, N., et al. 2010, A\&A, 518, L2

Portegies Zwart, S. F. \& van den Heuvel, E. P. J. 2016, MNRAS, 456, 3401

Prinja, R. K. \& Schild, H. 1991, A\&A, 249, 428

Reguitti, A., Pastorello, A., Pignata, G., et al. 2019, MNRAS, 482, 2750

Richardson, N. D. \& Mehner, A. 2018, RNAAS, 2, 121

Riebel, D., Srinivasan, S., Sargent, B., \& Meixner, M. 2012, ApJ, 753, 71

Riechers, D. A., Leung, T. K. D., Ivison, R. J., et al. 2017, ApJ, 850, 1

Rieke, G. H., Young, E. T., Engelbracht, C. W., et al. 2004, ApJS, 154, 25

Ruffle, P. M. E., Kemper, F., Jones, O. C., et al. 2015, MNRAS, 451, 3504

Schmutz, W., Leitherer, C., Hubeny, I., et al. 1991, ApJ, 372, 664

Schneider, F. R. N., Sana, H., Evans, C. J., et al. 2018, Science, 359, 69

Silvia, D. W., Smith, B. D., \& Shull, J. M. 2010, ApJ, 715, 1575

Silvia, D. W., Smith, B. D., \& Shull, J. M. 2012, ApJ, 748, 12

Smith, N. 2007, AJ, 133, 1034

Smith, N. 2010, MNRAS, 402, 145

Smith, N. 2014, ARA\&A, 52, 487

Smith, N., Chornock, R., Li, W., et al. 2008a, ApJ, 686, 467

Smith, N., Foley, R. J., \& Filippenko, A. V. 2008b, ApJ, 680, 568

Smith, N., Li, W., Foley, R. J., et al. 2007, ApJ, 666, 1116

Sodroski, T. J., Dwek, E., Hauser, M. G., \& Kerr, F. J. 1987, ApJ, 322, 101

Srinivasan, S., Boyer, M. L., Kemper, F., et al. 2016, MNRAS, 457, 2814

Srinivasan, S., Meixner, M., Leitherer, C., et al. 2009, AJ, 137, 4810

Stahl, O. \& Wolf, B. 1987, A\&A, 181, 293

Stahl, O., Wolf, B., Klare, G., Juettner, A., \& Cassatella, A. 1990, A\&A, 228, 379 
Stahl, O., Wolf, B., Leitherer, C., et al. 1984, A\&A, 140, 459 Stahl, O., Wolf, B., \& Zickgraf, F. J. 1987, A\&A, 184, 193

Stahl, O., Wolf, B., Zickgraf, F. J., et al. 1983, A\&A, 120, 287

Sugerman, B. E. K., Ercolano, B., Barlow, M. J., et al. 2006, Science, 313, 196

Sundqvist, J. O., Björklund, R., Puls, J., \& Najarro, F. 2019, A\&A, 632, A126

Szeifert, T., Stahl, O., Wolf, B., et al. 1993, A\&A, 280, 508

Tamura, Y., Mawatari, K., Hashimoto, T., et al. 2019, ApJ, 874, 27

Tartaglia, L., Elias-Rosa, N., Pastorello, A., et al. 2016, ApJ, 823, L23

Temim, T., Dwek, E., Tchernyshyov, K., et al. 2015, ApJ, 799, 158

Todini, P. \& Ferrara, A. 2001, MNRAS, 325, 726

Trundle, C., Kotak, R., Vink, J. S., \& Meikle, W. P. S. 2008, A\&A, 483, L47

Tubbesing, S., Kaufer, A., Stahl, O., et al. 2002, A\&A, 389, 931

Umana, G., Buemi, C. S., Trigilio, C., et al. 2009, ApJ, 694, 697

Umana, G., Buemi, C. S., Trigilio, C., Leto, P., \& Hora, J. L. 2010, ApJ, 718, 1036

Umana, G., Ingallinera, A., Trigilio, C., et al. 2012, MNRAS, 427, 2975

Urbaneja, M. A., Kudritzki, R. P., Gieren, W., et al. 2017, AJ, 154, 102

Vamvatira-Nakou, C., Hutsemékers, D., Royer, P., et al. 2015, A\&A, 578, A108

Vamvatira-Nakou, C., Hutsemékers, D., Royer, P., et al. 2013, A\&A, 557, A20

van Aarle, E., van Winckel, H., Lloyd Evans, T., et al. 2011, A\&A, 530, A90

van der Marel, R. P. \& Cioni, M.-R. L. 2001, AJ, 122, 1807

Van Dyk, S. D. \& Matheson, T. 2012, ApJ, 746, 179

van Gelder, M. L., Kaper, L., Japelj, J., et al. 2020, A\&A, 636, A54

van Genderen, A. M. 2001, A\&A, 366, 508

van Genderen, A. M. \& Sterken, C. 1996, A\&A, 308, 763

van Genderen, A. M. \& Sterken, C. 1999, A\&A, 349, 537

van Genderen, A. M., Sterken, C., \& de Groot, M. 1998, A\&A, 337, 393

van Loon, J. T., Oliveira, J. M., Gordon, K. D., et al. 2010, AJ, 139, 68

Vink, J. S. \& de Koter, A. 2002, A\&A, 393, 543

Vink, J. S., de Koter, A., \& Lamers, H. J. G. L. M. 1999, A\&A, 350, 181

Virtanen, P., Gommers, R., Oliphant, T. E., et al. 2020, Nature Methods, 17, 261

Voors, R. H. M., Waters, L. B. F. M., Morris, P. W., et al. 1999, A\&A, 341, L67

Walborn, N. R., Barbá, R. H., \& Sewiło, M. M. 2013, AJ, 145, 98

Walborn, N. R., Gamen, R. C., Morrell, N. I., et al. 2017, AJ, 154, 15

Walborn, N. R., Stahl, O., Gamen, R. C., et al. 2008, ApJ, 683, L33

Weis, K. 2003, A\&A, 408, 205

Weis, K., Chu, Y. H., Duschl, W. J., \& Bomans, D. J. 1997, A\&A, 325, 1157

Weis, K. \& Duschl, W. J. 2002, A\&A, 393, 503

Weis, K., Duschl, W. J., \& Bomans, D. J. 2003, A\&A, 398, 1041

Wesson, R., Barlow, M. J., Matsuura, M., \& Ercolano, B. 2015, MNRAS, 446, 2089

Whitney, B. A., Sewilo, M., Indebetouw, R., et al. 2008, AJ, 136, 18

Wolf, B. 1989, A\&A, 217, 87

Wolf, B., Stahl, O., de Groot, M. J. H., \& Sterken, C. 1981, A\&A, 99, 351

Wolf, B., Stahl, O., \& Seifert, W. 1987, A\&A, 186, 182

Zickgraf, F. J., Humphreys, R. M., Lamers, H. J. G. L. M., et al. 1996a, A\&A, 315,510

Zickgraf, F. J., Kovacs, J., Wolf, B., et al. 1996b, A\&A, 309, 505

Zickgraf, F. J., Wolf, B., Stahl, O., Leitherer, C., \& Appenzeller, I. 1986, A\&A, 163,119 


\section{Appendix A: Table of stellar and nebular \\ parameters}

We use stellar parameters collected from the literature. Only values corresponding to the quiescent state of the stars were taken into account. To compensate for the lack of error estimates, we take the mean value and the corresponding standard deviation when more than one estimate is available. In this case all the relevant works are mentioned. We also include information on the nebula size when known from the literature and from this work.

\section{Appendix B: IR images and SEDs}

Flux density distribution plots of individual sources and selected space telescope images are provided here. The photometric data are corrected for interstellar dust extinction, using the extinction curve from Fitzpatrick (1999), E(B-V) as in Table A.1 and $R_{V}=3.1$. For RMC 143 an $R_{V}=4.0$ was adopted as previously determined by Agliozzo et al. (2019). The images are useful for assessing the confusion and thus the reliability of the photometry in each band. The field-of-view in each image is $240^{\prime \prime} \times 240^{\prime \prime}$. The position of the source is highlighted with a cyan cross-hair, which is rotated accordingly with the sky projection. The colourscale is squared-root in order to highlight the local environment around each source. 

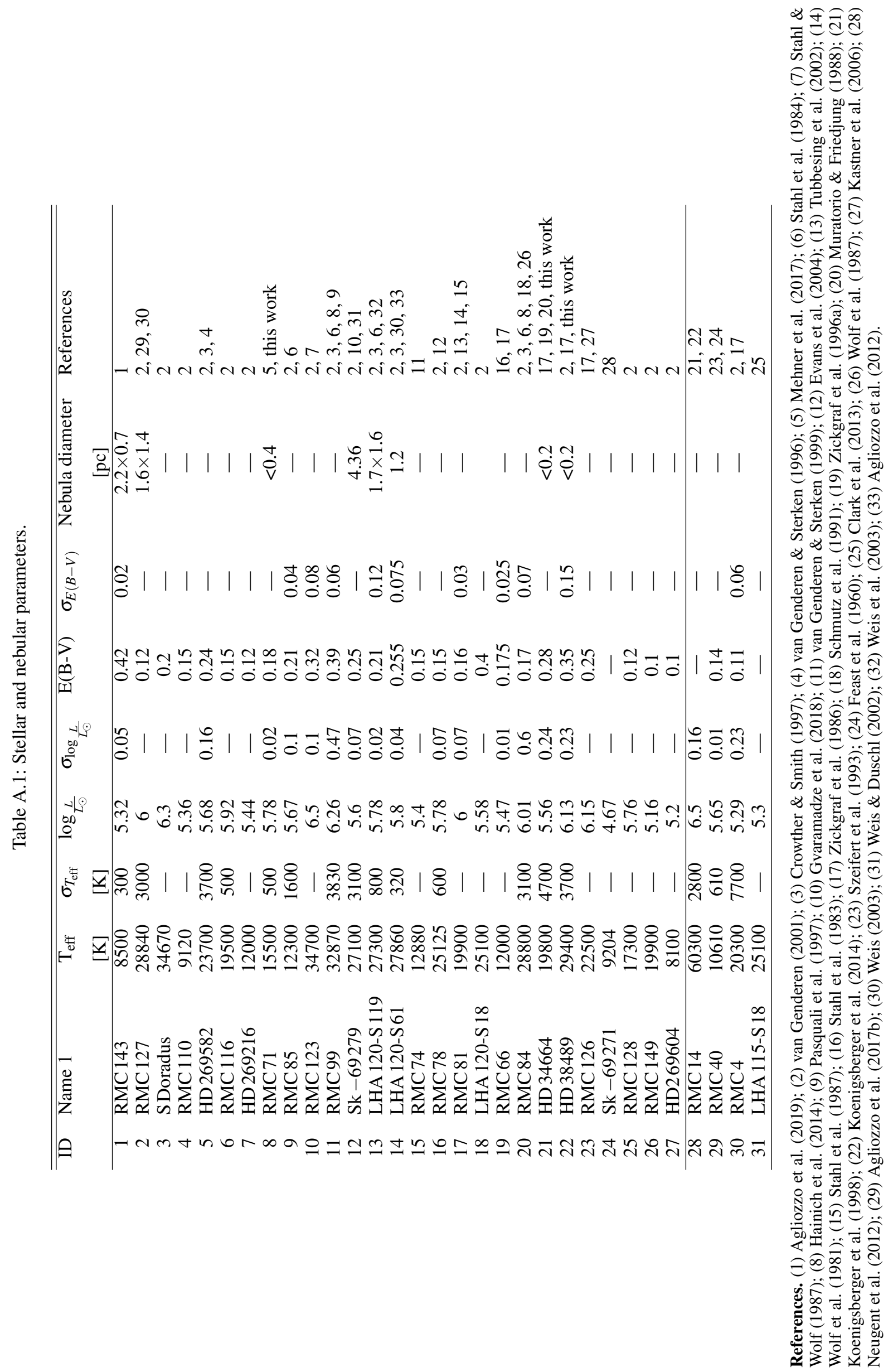

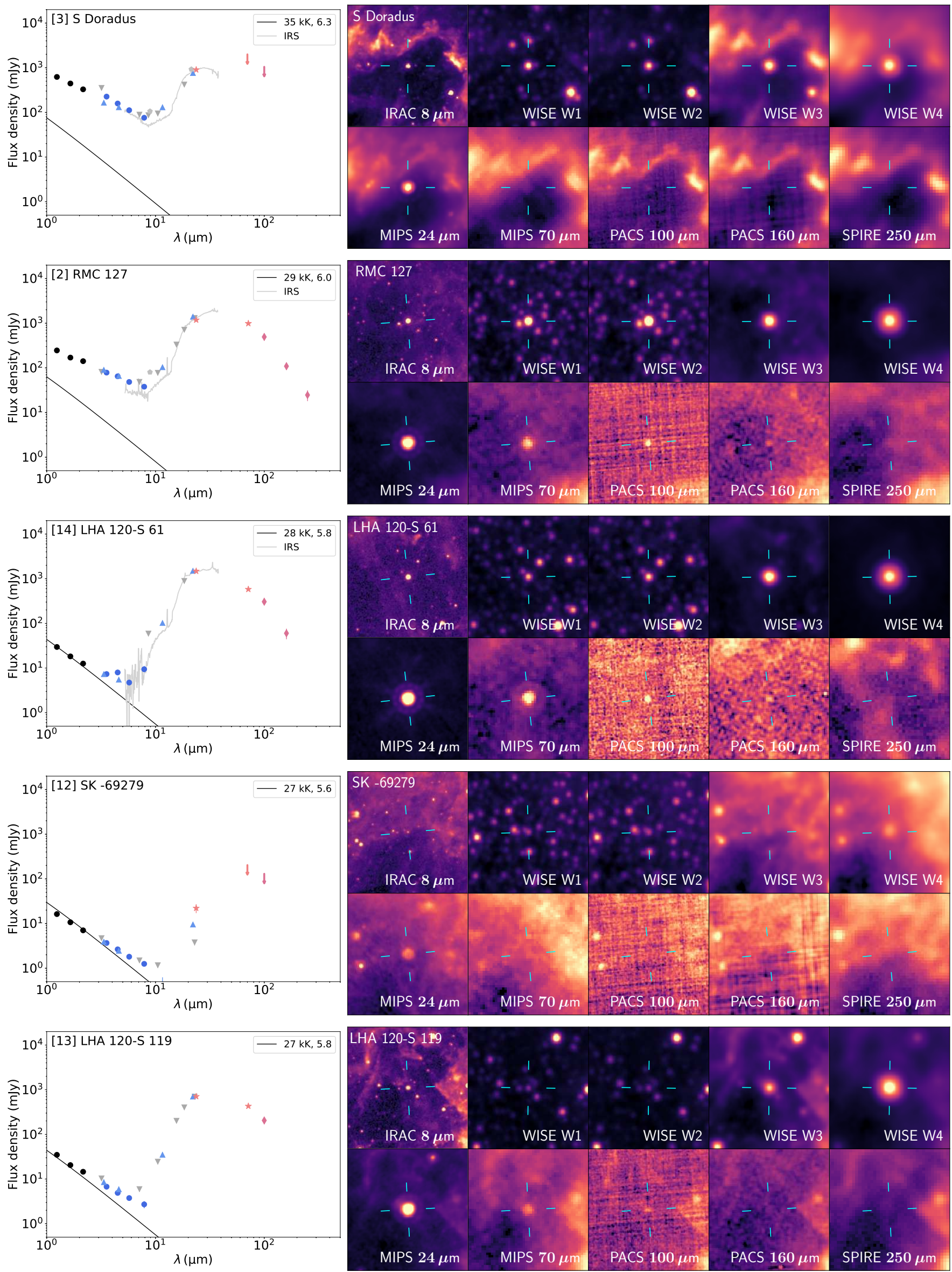

Fig. B.1: Confirmed and candidate LBVs with a SED characterised by a free-free continuum from their stellar wind and by a thermal continuum due to dust in their circumstellar ejecta (see Class 1a sources in Sect. 4). The legend for the photometric points can be found in Fig. 5 of Sect. 4. Article number, page 25 of 37 

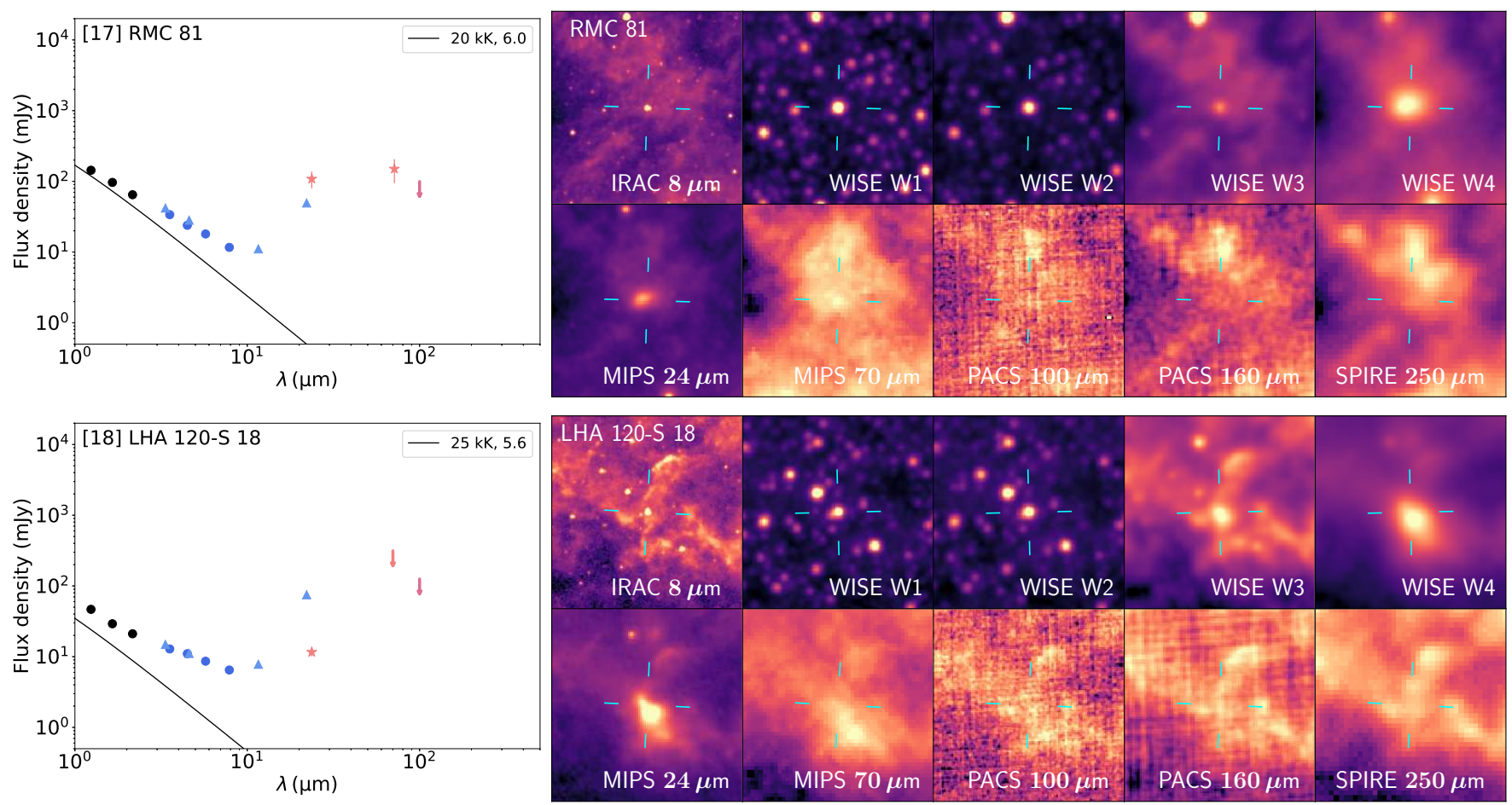

Fig. B.1 continued. 

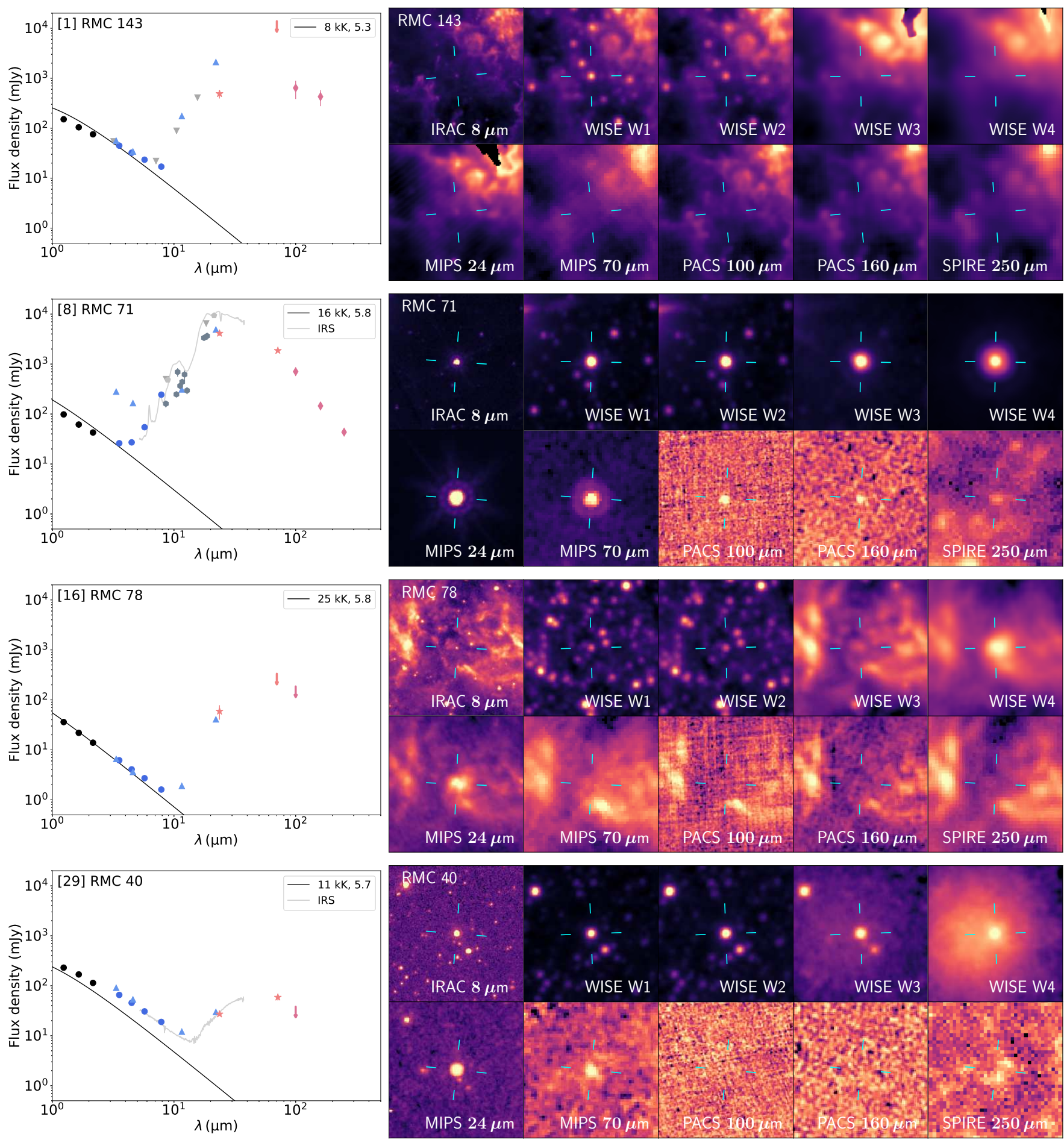

Fig. B.2: Confirmed and candidate LBVs with a SED characterised by a thermal continuum due to dust in their circumstellar ejecta and relatively weak or absent free-free continuum from their stellar wind (see Class $1 \mathrm{~b}$ sources in Sect. 4). The legend for the photometric points can be found in Fig. 5 of Sect. 4 

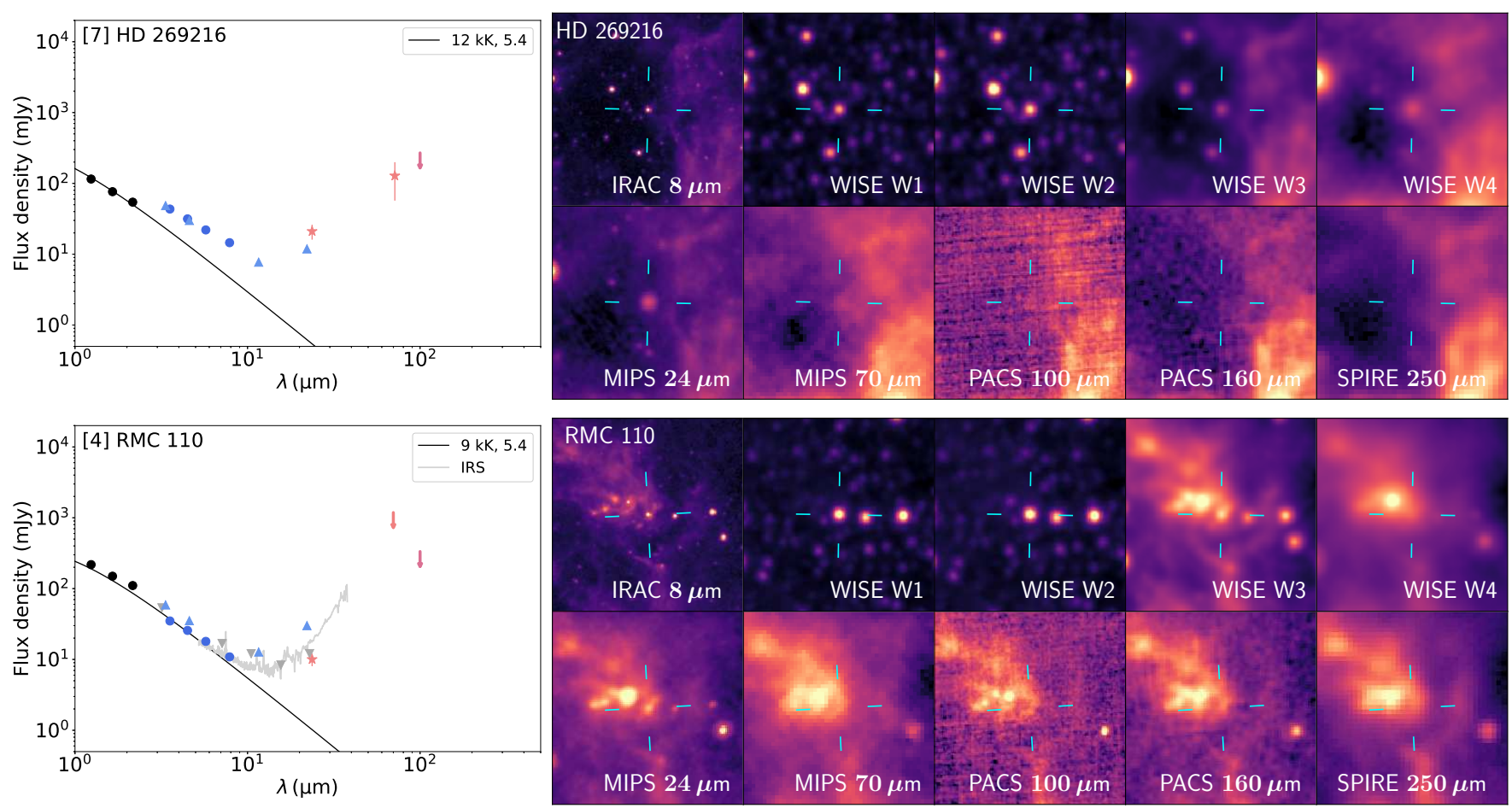

Fig. B.2 continued 

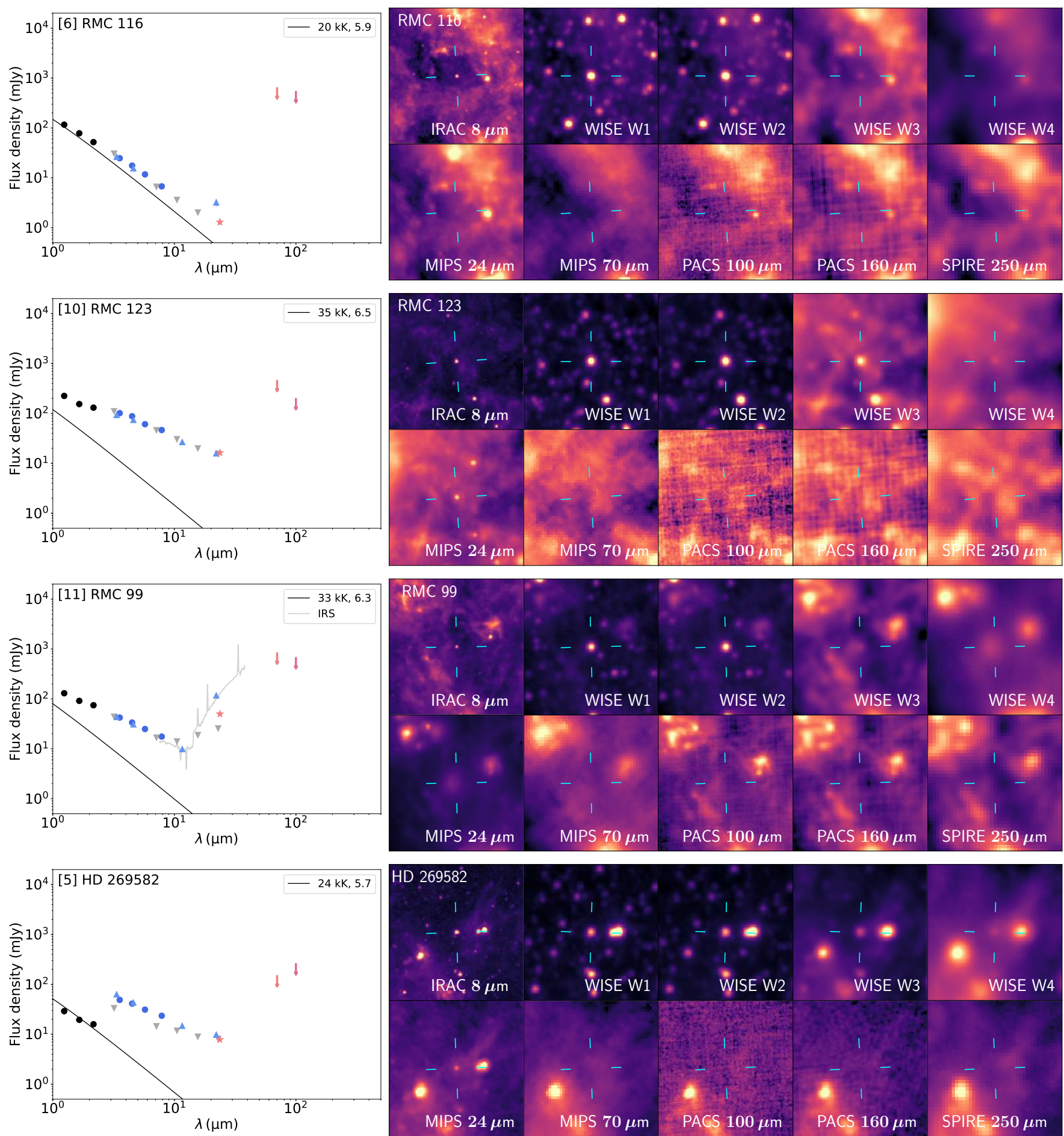

Fig. B.3: Confirmed and candidate LBVs with a SED dominated by free-free continuum up to $24 \mu \mathrm{m}$ and by confusion at longer wavelengths (Class 2 sources in Sect. 47. For HD 269582 the jump between 2MASS and other photometry is due to real source variability. The legend for the photometric points can be found in Fig. 5 of Sect. 4. 

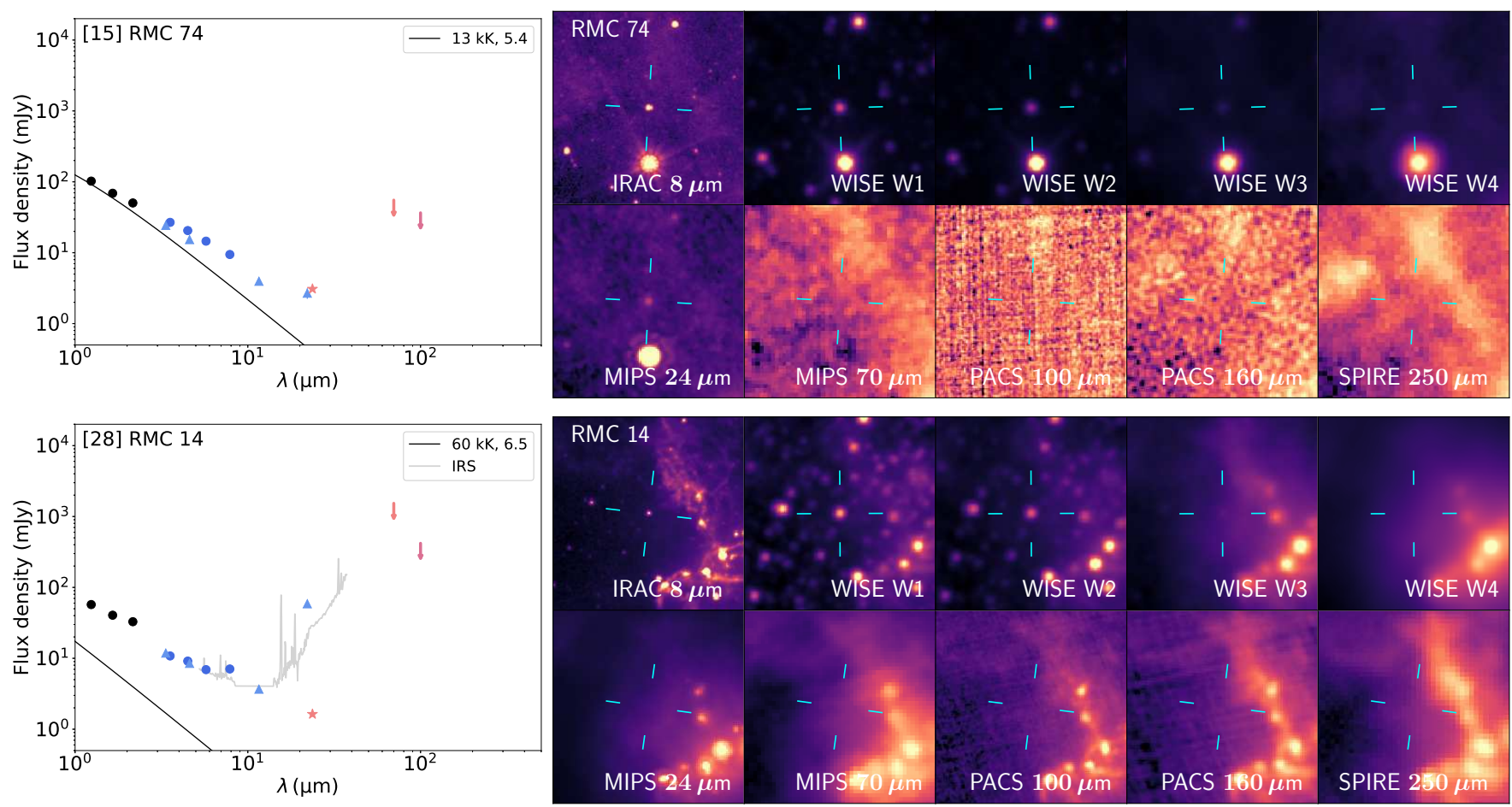

Fig. B.3 continued. We note that photometry for RMC 14 has not been extinction corrected due to no $A_{\mathrm{V}}$ value in the literature.
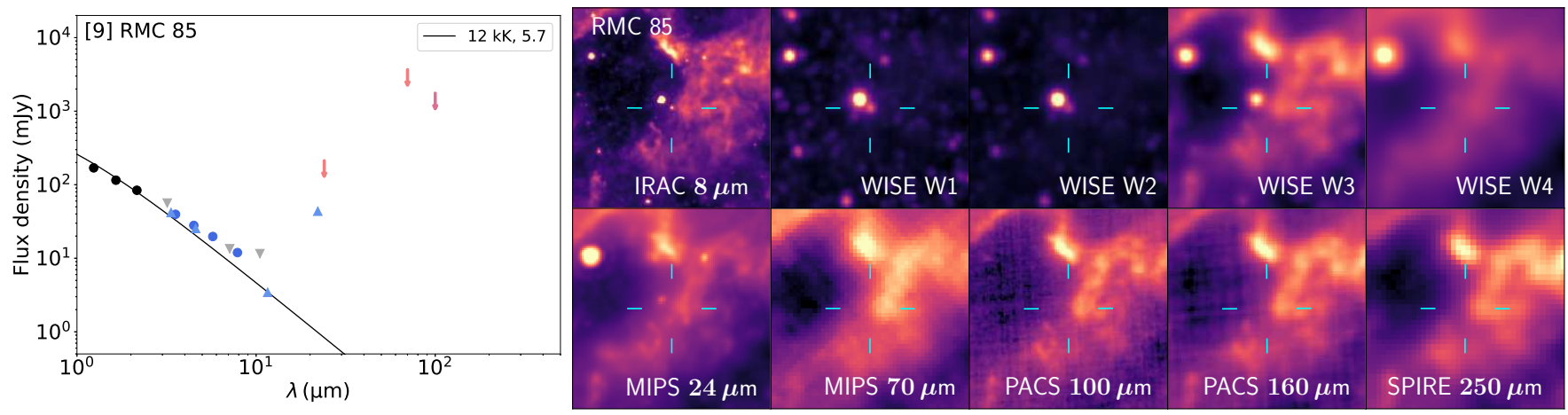

Fig. B.4: Unclassified LBV RMC 85. No reliable photometry could be obtained longward of $20 \mu \mathrm{m}$ due to confusion with bright ISM dust. The legend for the photometric points can be found in Fig. 5 of Sect. 4 

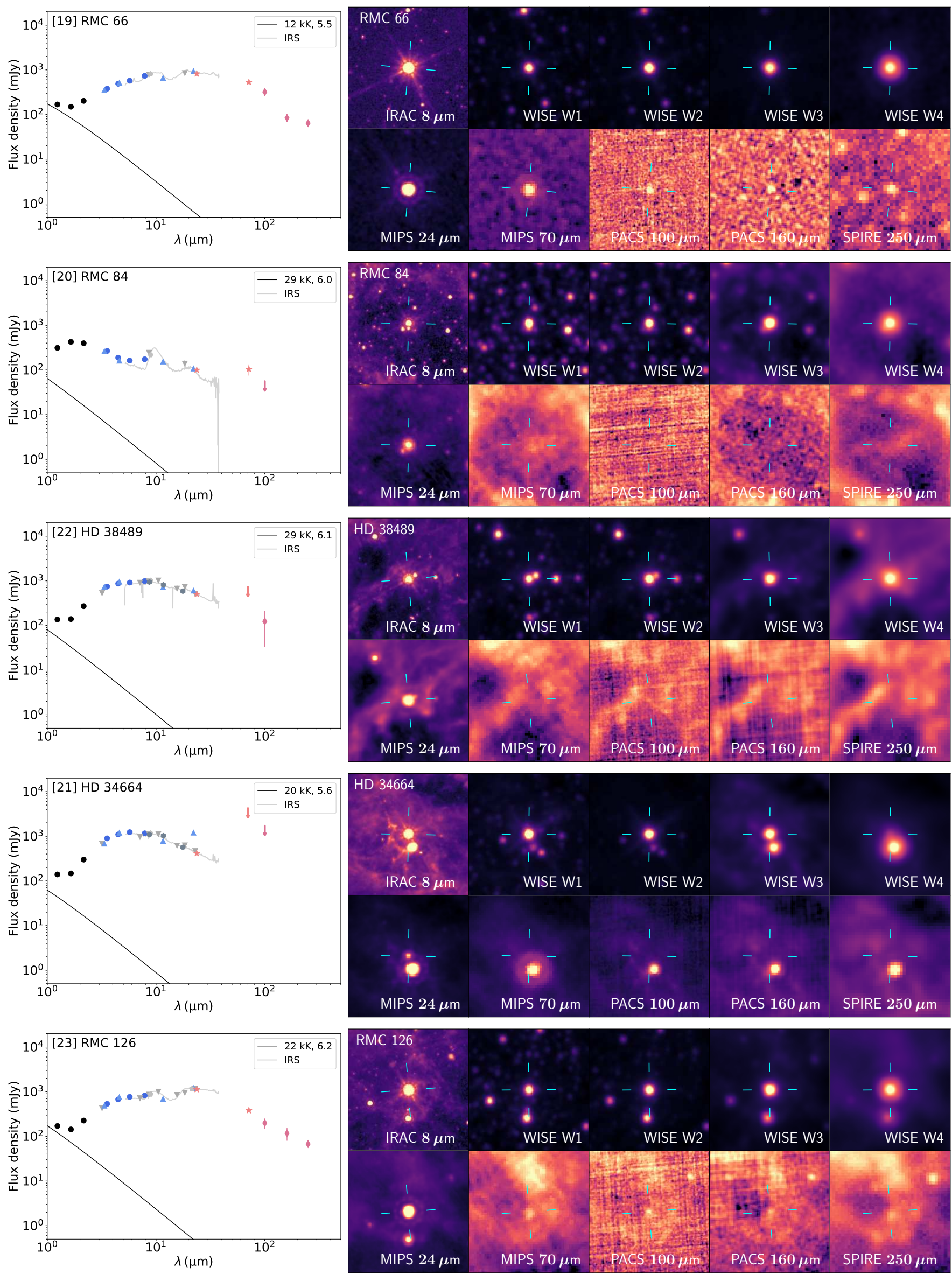

Fig. B.5: $\mathrm{sgB}[\mathrm{e}]$ stars as in literature (see Class 3 sources in Sect. 44. The legend for the photometric points can be found in Fig. 5 of Sect. 4 

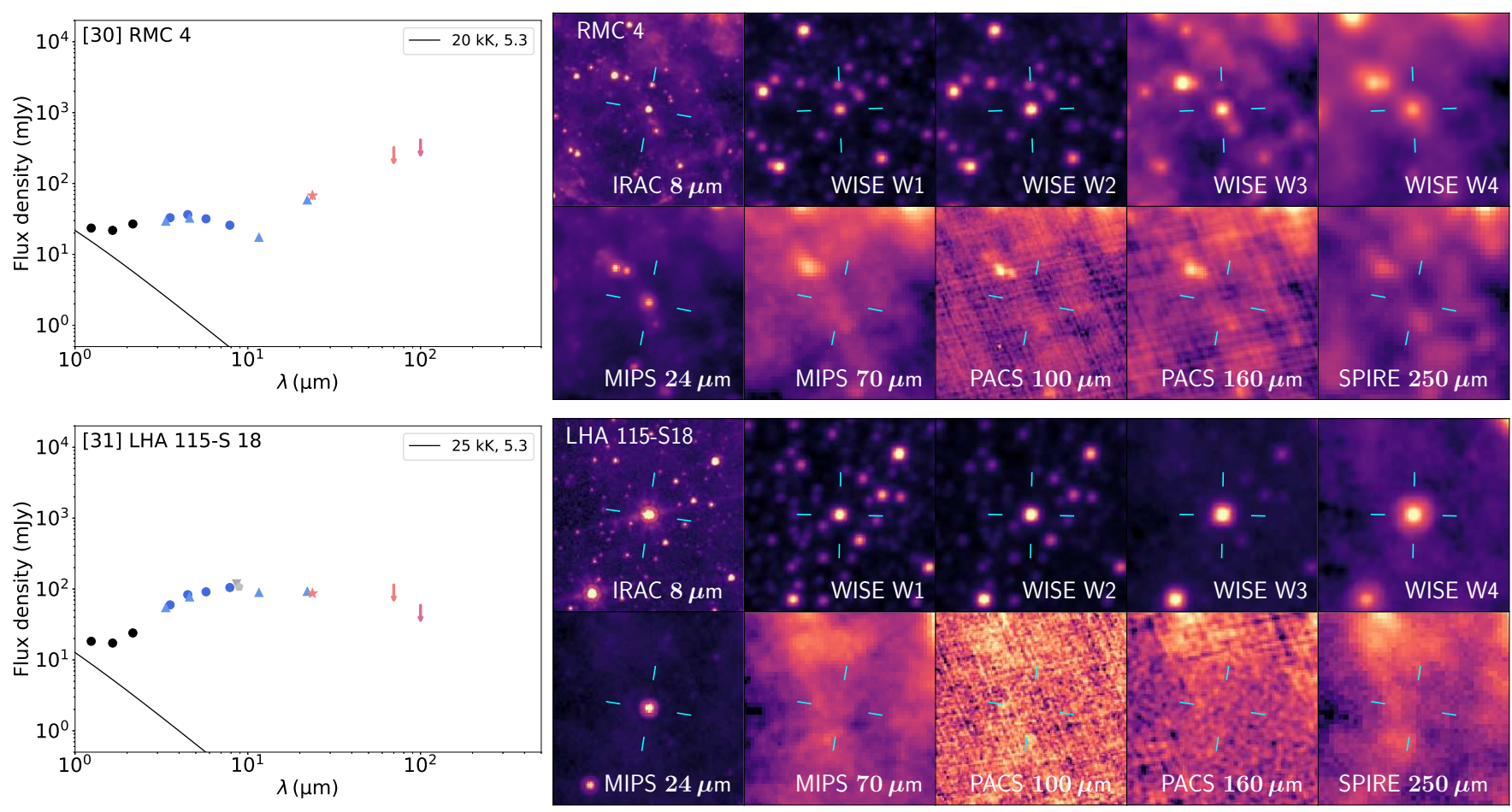

Fig. B.5 continued. We note that photometry for LHA 115-S 18 has not been extinction corrected due to no $A_{\mathrm{V}}$ value in the literature. 

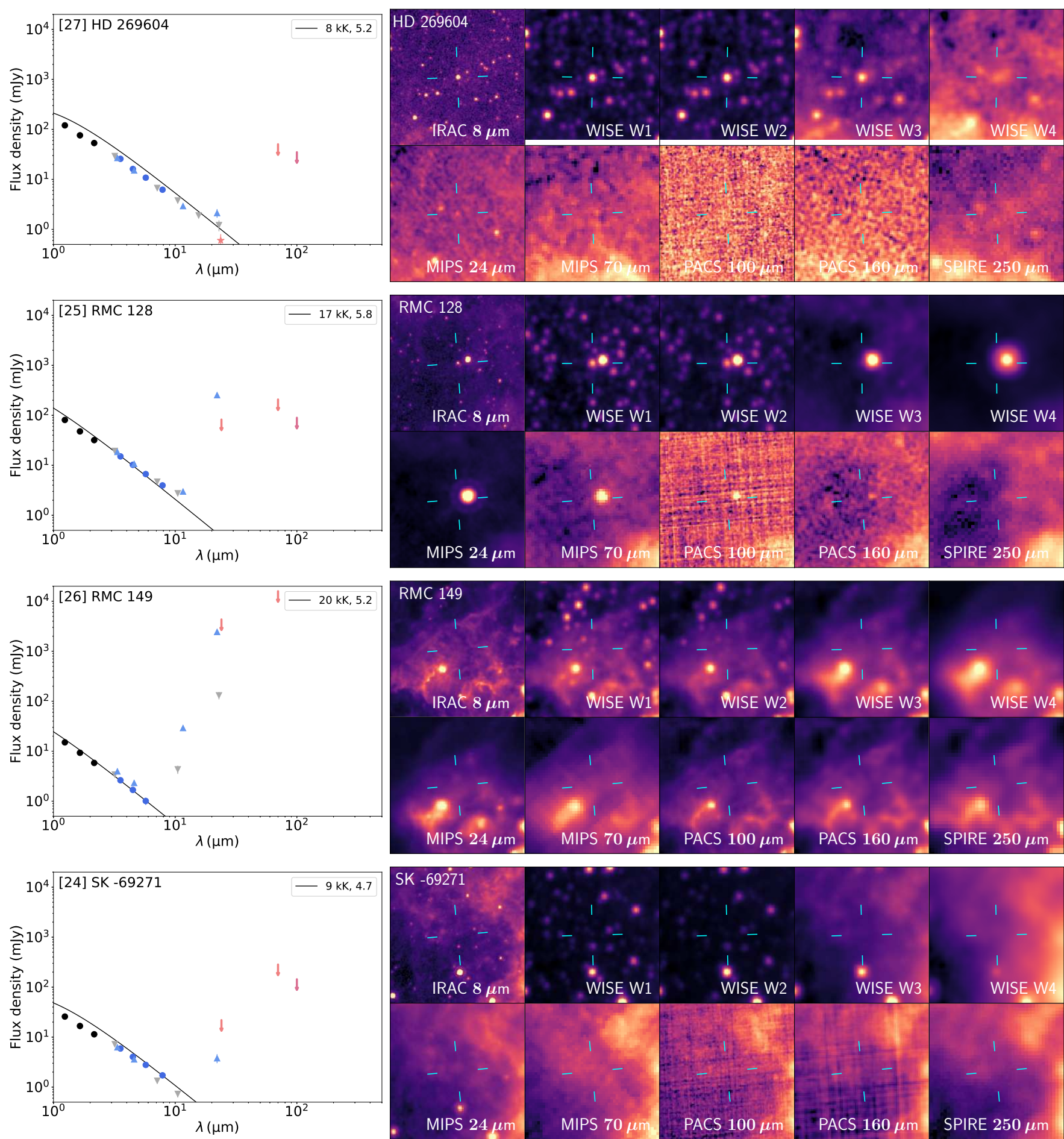

Fig. B.6: Featureless sources with no recognised LBV membership (see Class 4 sources in Sect. 47. We note that photometry for Sk -69271 has not been extinction corrected due to no $A_{\mathrm{V}}$ value in the literature. The legend for the photometric points can be found in Fig. 5 of Sect. 4 


\section{Appendix C: Tables of infrared photometry}

In this appendix the adopted photometry for all sources from the near-infrared to far-infrared are provided. In the following tables all photometry is provided as frequency flux density in units of mJy. Where catalogue values were provided as magnitudes, these have been converted to flux densities using zero point (zero magnitude) fluxes which are indicated in the table header.

All 2MASS photometry comes from the 2MASS Point Source Catalogue (Cutri et al. 2003), and were converted to flux densities using the zero point fluxes from $\$ 6.4 \mathrm{a}$ of the corresponding Explanatory Supplemen 7 . AKARI N3, S7, S11, L15 and L24 photometry come from the AKARI-LMC Point-source catalog (Kato et al. 2012), and were converted to flux densities using the zero point fluxes from Kato et al. (2012) (which, although of low precision, are the logical choice to use, as they explain that the photometry was first calibrated into units of Jy and then converted to the magnitudes in the catalogue using these zero point fluxes). AKARI S9W and L18W photometry come from the AKARI/IRC mid-infrared all-sky Survey (Ishihara et al. 2010), originally in Jy units. WISE photometry almost exclusively comes from The AllWISE data Release (updated version, 16-Feb-2021) (Cutri et al. 2021). WISE W4 values for RMC 116 (ID 6) and Sk-69271 (ID 24) come from The WISE All-Sky data Release (Cutri et al. 2012) as they are not detected in ALLWISE (both are faint and hard to distinguish from the background emission). WISE magnitudes were converted to flux densities using zero point fluxes for a $F_{v} \propto v^{-2}$ spectrum from §IV.4.i.1 of the Explanatory Supplement to the WISE AllSky Data Release Product ${ }^{8}$. All MSX photometry comes from the MSX Point Source Catalog Version 2.3 (Egan et al.|2003), originally in Jy units.

The Spitzer and Herschel photometry are discussed in the main text, with some values newly extracted in this work.

For completeness, catalogue values that we have identified as incorrect are included, with their value suffixed by a flag character. A ' $c$ ' denotes that the value is most likely affected by confusion with nearby bright emission, and a ' $r$ ' denotes that the value is likely affected by the source being resolved. These were assessed with the aid of the flux density distributions and images in Appendix B

\footnotetext{
7 https://old.ipac.caltech.edu/2mass/releases/ allsky/doc/sec6_4a.html

8 https://wise2.ipac.caltech.edu/docs/release/ allsky/expsup/sec4_4h.html
}

Article number, page 34 of 37 


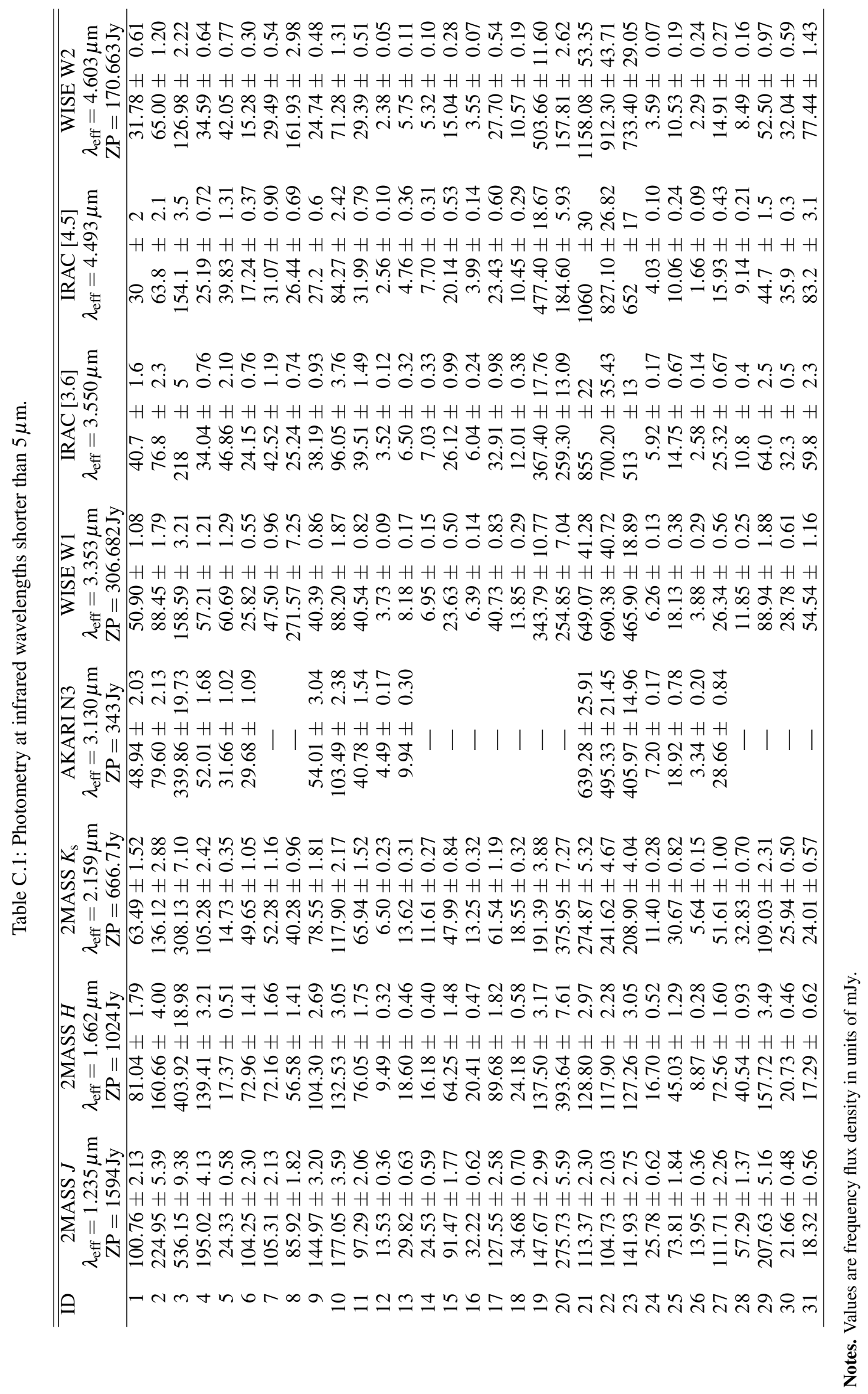




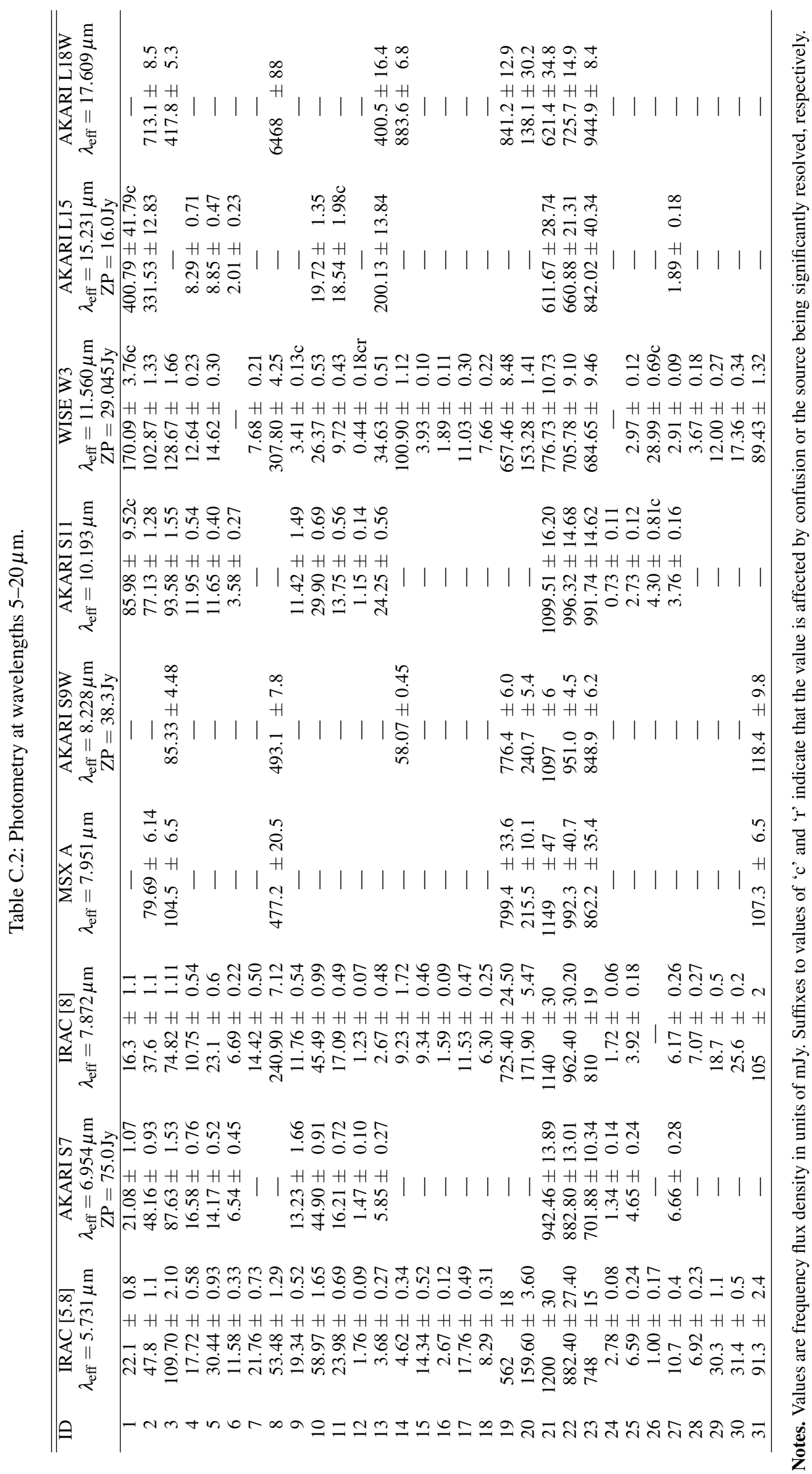




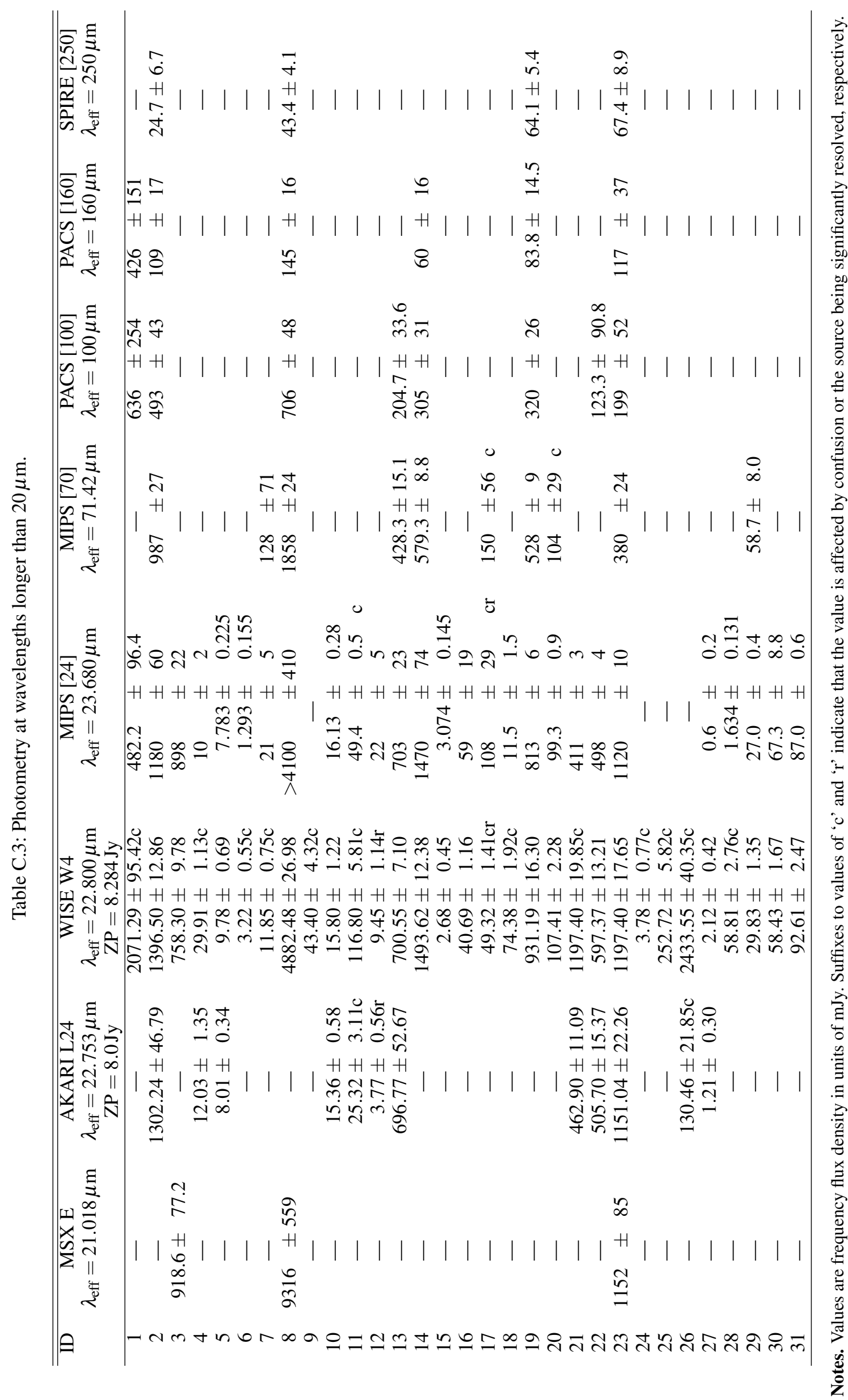

